\title{
Heterofunctionalized Cavitands by Macrocyclization of Sequence-Defined Foldamers
}

\author{
Joseph W. Meisel,* Chunhua Hu, and Andrew D. Hamilton*
}

\author{
Supporting Information
}

\section{General Methods}

\subsection{Solvents and Reagents}

Reagents were purchased from Sigma Aldrich (St. Louis, MO) or Oakwood Products (Estill, NC) and used without further purification. Reactions were carried out under an argon atmosphere in oven-dried glassware. Anhydrous solvents dimethylformamide (DMF), dichloromethane $\left(\mathrm{CH}_{2} \mathrm{Cl}_{2}\right)$, tetrahydrofuran (THF), dioxane, and methanol (MeOH) were purchased from Sigma Aldrich or Fisher Scientific and used without further purification.

\subsection{Spectroscopy}

${ }^{1} \mathrm{H}$ and ${ }^{13} \mathrm{C}$ NMR spectroscopy was carried out on a Bruker Ascend-400 MHz (100 MHz carbon) or a Bruker AVANCE-III-600 MHz (150 MHz carbon) spectrometer running TopSpin ${ }^{\mathrm{TM}}$ software. Chemical shifts are given in parts per million (ppm) and are referenced against tetramethylsilane (TMS) or residual solvent internal standards. Coupling constants $(J)$ are given in Hertz $(\mathrm{Hz})$. Multiplicity is abbreviated as follows: $\mathrm{s}=$ singlet, $\mathrm{br}=$ broad, $\mathrm{d}=$ doublet, $\mathrm{dd}=$ doublet of doublet, $\mathrm{t}=$ triplet, $\mathrm{dt}=$ doublet of triplet, $\mathrm{q}=$ quartet, $\mathrm{dq}=$ doublet of quartet, $\mathrm{qn}=$ quintet, sept $=$ septet, $\mathrm{m}=$ multiplet. Compound names are generated following IUPAC nomenclature using ChemDraw ${ }^{\mathrm{TM}}$ Professional (CambridgeSoft). High resolution mass spectra (HRMS) were acquired on an Agilent 6224 Accurate-Mass TOF LC/MS using an atmospheric pressure chemical ionization (ESI) source or electrospray ionization (ESI) source. Oligomer and macrocycle mass spectra were acquired on a Bruker ultrafleXtreme MALDI-TOF/TOF mass spectrometer using a 2,5-dihydroxybenzoic acid (DHB) matrix. X-ray structural determinations were performed on a Bruker AXS SMART APEXII single crystal diffractometer equipped with an Oxford Cryosystems 700 plus cooler.

\subsection{Column Chromatography}

Compound purification by normal phase automated flash chromatography was carried out on a Biotage ${ }^{\circledR}$ Isolera $^{\mathrm{TM}}$ instrument using SNAP Ultra columns $(25 \mu \mathrm{m}$ spherical silica, $10 \mathrm{~g}, 25 \mathrm{~g}$, or $50 \mathrm{~g}$ columns) and applying a gradient elution of hexanes and ethyl acetate. Compound purification by reverse phase semipreparative UHPLC is performed on a Thermo Scientific ${ }^{\mathrm{TM}}$ UltiMate 3000 system using a Waters $^{\mathrm{TM}}$ SunFire ${ }^{\circledR}$ C18 OBD Prep $(5 \mu \mathrm{m}, 19 \mathrm{~mm} \times 150 \mathrm{~mm})$ column using water, acetonitrile, and methanol mobile phases containing $0.1 \%$ formic acid using a flow rate of $3.0 \mathrm{~mL} \mathrm{~min}^{-1}$ and photodiode array detection at $\lambda=220-280 \mathrm{~nm}$.

\section{General Procedures}

\subsection{General Procedure (a) - Alkylation of methyl 5-formyl-4-hydroxy-2-methoxybenzoate by} Williamson ether synthesis.

To a stirred solution of methyl 5-formyl-4-hydroxy-2-methoxybenzoate (1) in dimethylformamide (10 $\mathrm{mL}$ ) was added potassium carbonate $(2.0 \mathrm{eq})$ and alkyl halide $(1.5 \mathrm{eq})$ and reaction mixture stirred at 
$50{ }^{\circ} \mathrm{C}$ until complete as indicated by consumption of starting material (monitored by TLC). The reaction mixture was then poured into ethyl acetate $(100 \mathrm{~mL})$ and the organic layer was washed with 1 M sodium bisulfate $(2 \times 75 \mathrm{~mL}), 5 \%$ sodium bicarbonate $(3 \times 75 \mathrm{~mL})$ and brine $(1 \times 75 \mathrm{~mL})$, then filtered over celite and magnesium sulfate and concentrated in vacuo. The product was purified by recrystallization from methylene chloride and hexane.

\subsection{General Procedure (b) - Saponification of methyl 5-formyl-4-alkoxy-2-methoxybenzoate.}

To a 0.2 M solution of methyl 5-formyl-4-alkoxy-2-methoxybenzoate $\mathbf{2 a - h}$ in methanol/tetrahydrofuran (1:1) was added $2 \mathrm{M}$ aqueous lithium hydroxide (10 eq) and reaction stirred at $50{ }^{\circ} \mathrm{C}$ until complete as indicated by consumption of starting material (monitored by TLC). The reaction mixture was then concentrated in vacuo, acidified with $2 \mathrm{M}$ aqueous hydrochloric acid, and extracted into ethyl acetate (2 x $75 \mathrm{~mL}$ ). The combined organic phases were washed with brine, filtered over celite and magnesium sulfate, and concentrated in vacuo.

\subsection{General Procedure (c) - Reductive carbamoylation of benzaldehydes 3a-h.}

To a solution of 5-formyl-4-alkoxy-2-methoxybenzoic acid 3a-h and 9-fluorenylmethyl carbamate (1.0 eq) in acetonitrile $(30 \mathrm{~mL})$ was added trifluoroacetic acid $(2.0 \mathrm{eq})$ and triethylsilane $(3.0 \mathrm{eq})$ and stirred at $50{ }^{\circ} \mathrm{C}$ until complete (4-16 h, monitored by TLC). The reaction mixture was then concentrated in vacuo and the residue was purified by flash chromatography (ethyl acetate/hexanes) to give compounds 4a-h.

\subsection{General Procedure (d) - Solid phase synthesis of MAMBA oligoamides.}

MAMBA tetramers and pentamers were prepared on 2-chlorotrityl chloride polystyrene resin (Manufacturer) with $1.46 \mathrm{mmol} / \mathrm{g}$ loading capacity. A typical reaction is performed on a $30 \mu \mathrm{mol}$ scale. Between each step the resin is washed with dichloromethane (3 tube volumes). The resin was swelled for 10 minutes in dichloromethane in a solid phase extraction tube (Sigma Aldrich) and then loaded by adding the $\mathrm{C}$-terminal monomer (Fmoc-MmbX-OH, 3.0 equivalents) and diisopropylethylamine (8.0 equivalents) in dichloromethane for 1-2 h. Extension of the oligomer chain was achieved by deprotection of the Fmoc-protected N-terminus with piperidine (20\% in DMF, 2 x 5 min) followed by coupling of the next monomer by addition of Fmoc-MmbX-OH (3.0 equivalents), diisopropylethylamine (8.0 equivalents), and HBTU (3.0 equivalents) in dichloromethane for 1-2 h. After iterative steps of Fmoc deprotection and monomer coupling, the final $\mathrm{N}$-terminal Fmoc protecting group was deprotected and the side chain-protected oligomer was cleaved from the resin with 1,1,1,3,3,3-hexafluoro-2-propanol (HFIP, 20\% in dichloromethane, 3 x $5 \mathrm{~min}$ ). The filtrate was collected, combined, and concentrated in vacuo. The side chain-protected oligomers were cyclized without further purification.

\subsection{General Procedure (e) - Solution phase cyclization of MAMBA oligoamides.}

Side chain-protected oligomers with the formula $\mathrm{H}-(\mathrm{MmbX})_{4}-\mathrm{OH}$ or $\mathrm{H}-(\mathrm{MmbX})_{5}-\mathrm{OH}$ and diisopropylethylamine (20 eq) were dissolved in 10\% dimethylformamide in dichloromethane and stirred vigorously. (Benzotriazol-1-yloxy)tripyrrolidinophosphonium hexafluorophosphate (PyBop, 5 eq) was added and the reaction mixture was stirred $16 \mathrm{~h}$ at room temperature, after which the reaction mixture was concentrated in vacuo. Water $\left(25 \mathrm{~mL}, 0{ }^{\circ} \mathrm{C}\right)$ is added to the residue and the precipitate is collected by centrifugation and dried in vacuo.

\subsection{General Procedure (f) - Global deprotection of MAMBA cavitands.}

To the side chain-protected cavitand is added a solution of trifluoroacetic acid, triisopropylsilane, and water $(18: 1: 1 \mathrm{v} / \mathrm{v} / \mathrm{v})$ and the reaction is stirred at room temperature for $1 \mathrm{~h}$. The reaction mixture is 
concentrated in vacuo and the residue is purified by UHPLC (methanol/water/acetonitrile) or by flash chromatography using ethyl acetate/hexanes or methanol/methylene chloride mobile phases.

\section{Procedures}

\subsection{Solution Phase Synthetic Procedures}

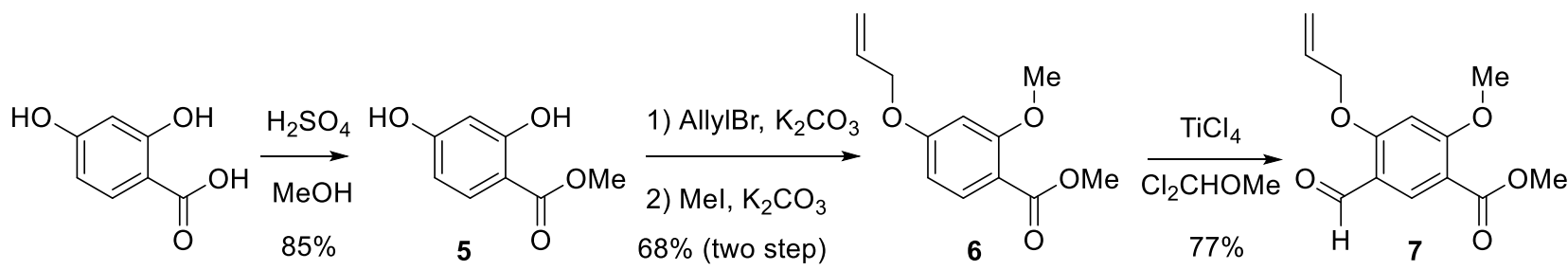

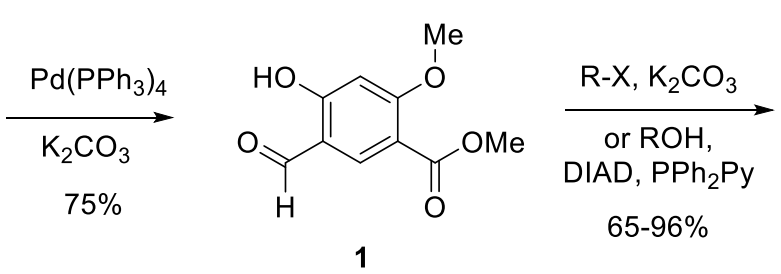<smiles>[R]Oc1cc(OC)c(C(=O)OC)cc1C=O</smiles><smiles>[R]Oc1cc(OC)c(C(=O)O)cc1C=O</smiles>

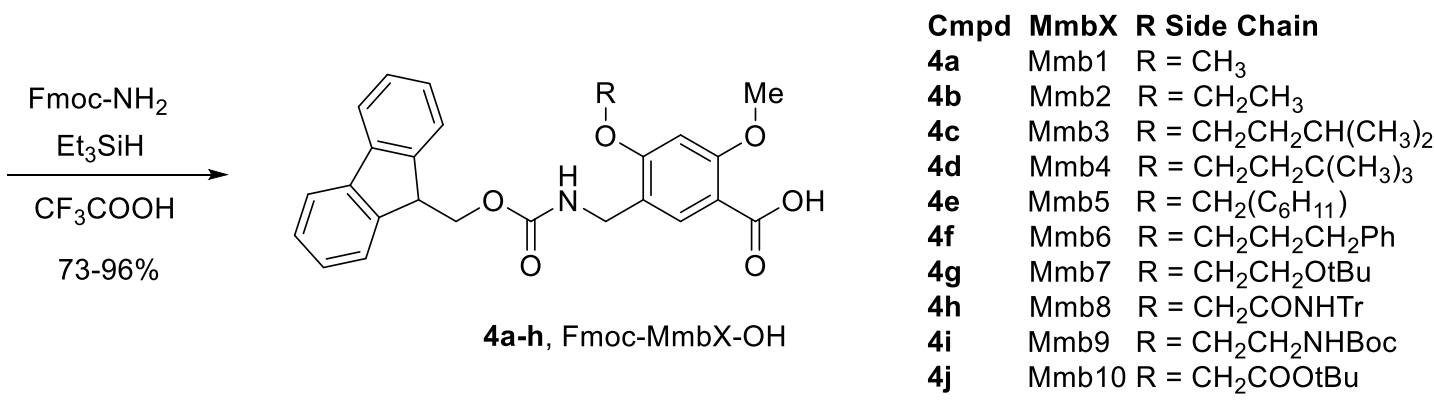

Figure S1: Synthesis of functionalized MAMBA monomers $\mathbf{4 a - h}$ from $\beta$-resorcylic acid.<smiles>COC(=O)c1ccc(O)cc1O</smiles>

methyl 2,4-dihydroxybenzoate (5)

To a stirred solution of 2,4-dihydroxybenzoic acid (50.0 g, $0.32 \mathrm{~mol})$ dissolved in methanol (260 $\mathrm{mL})$ was added sulfuric acid $(21 \mathrm{~mL}, 0.39 \mathrm{~mol})$ and brought to reflux for $4 \mathrm{~h}$. The reaction mixture was then concentrated in vaсио. The product is precipitated by the addition of cold water, filtered, and washed with cold water, and dried to give the title compound 5 as a white crystalline solid (46.1 g, 85\%). ${ }^{1} \mathrm{H}$ NMR (600 MHz, Chloroformd) $\delta 11.00(\mathrm{~s}, 1 \mathrm{H}), 7.76(\mathrm{~d}, J=8.6 \mathrm{~Hz}, 1 \mathrm{H}), 6.45-6.37(\mathrm{~m}, 2 \mathrm{H}), 5.32,4.79(\mathrm{~s}, 1 \mathrm{H}), 3.94(\mathrm{~s}, 3 \mathrm{H}) .{ }^{13} \mathrm{C} \mathrm{NMR}(151$ MHz, Chloroform- $d$ ) $\delta$ 170.38, 163.60, 161.88, 131.95, 107.85, 105.95, 103.15, 52.09. HRMS (ESI ${ }^{+}$): Calcd for $\mathrm{C}_{8} \mathrm{H}_{9} \mathrm{O}_{4}{ }^{+}[\mathrm{M}+\mathrm{H}]^{+}$169.0495, found 169.0498.<smiles>C=CCOc1ccc(C(=O)OC)c(OC)c1</smiles>

methyl 4-allyloxy-2-methoxybenzoate (6) 
To a stirred solution of methyl 2,4-dihydroxybenzoate 5 (46.0 g, $0.27 \mathrm{~mol})$ and potassium carbonate (41.6 g, $0.30 \mathrm{~mol})$ in acetone $(200 \mathrm{~mL})$ was added a solution of allyl bromide $(26.0 \mathrm{~mL} 0.30 \mathrm{~mol})$ in acetone $(100 \mathrm{~mL})$ dropwise over $1 \mathrm{~h}$ and then brought to reflux. Upon consumption of starting material (monitored by TLC) the reaction mixture was cooled, filtered over celite, and the filter cake was rinsed with acetone (200 $\mathrm{mL})$. To the combined filtrate was added potassium carbonate $(56.7 \mathrm{~g}, 0.41 \mathrm{~mol})$ and iodomethane $(25.6 \mathrm{~mL}, 0.41 \mathrm{~mol})$ and stirred at room temperature $48 \mathrm{~h}$. Upon completion of the reaction, the mixture was filtered over celite, the filter cake was rinsed with acetone $(200 \mathrm{~mL})$ and the combined filtrates were concentrated in vacuo. The title compound 6 was obtained by recrystallization from hexanes at $0{ }^{\circ} \mathrm{C}$ as a white crystalline solid $(41.4 \mathrm{~g}, 68 \%)$. ${ }^{1} \mathrm{H}$ NMR (400 MHz, Chloroform- $d$ ) $\delta 7.84(\mathrm{~d}, J=8.5 \mathrm{~Hz}, 1 \mathrm{H}), 6.57-6.45(\mathrm{~m}, 2 \mathrm{H}), 6.13-5.98(\mathrm{~m}, 1 \mathrm{H}), 5.49-$ $5.39(\mathrm{~m}, 1 \mathrm{H}), 5.32(\mathrm{dt}, J=10.5,1.4 \mathrm{~Hz}, 1 \mathrm{H}), 4.58(\mathrm{dt}, J=5.4,1.5 \mathrm{~Hz}, 2 \mathrm{H}), 3.87(\mathrm{~d}, J=12.2 \mathrm{~Hz}, 6 \mathrm{H}) .{ }^{13} \mathrm{C} \mathrm{NMR}$ (151 MHz, Chloroform- $d$ ) $\delta 166.11,163.23,161.31,133.82,132.55,118.25,112.41,105.22,99.67,68.98$, 55.99, 51.73. HRMS (ESI ${ }^{+}$: Calcd for $\mathrm{C}_{12} \mathrm{H}_{14} \mathrm{O}_{4} \mathrm{Na}^{+}[\mathrm{M}+\mathrm{Na}]^{+} 245.0784$, found 245.0787.

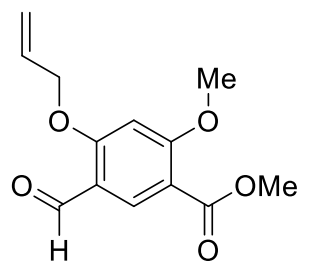

methyl 5-formyl-4-allyloxy-2-methoxybenzoate (7)

To a stirred solution of methyl 4-allyloxy-2-methoxybenzoate 6 (36.0 g, $0.16 \mathrm{~mol})$ and dichloromethyl methyl ether $(36.6 \mathrm{~mL}, 0.40 \mathrm{~mol})$ in dichloromethane $(150 \mathrm{~mL})$ at $0{ }^{\circ} \mathrm{C}$ was added dropwise titanium (IV) chloride $(53.3 \mathrm{~mL}, 0.49 \mathrm{~mol})$ in dichloromethane $(50 \mathrm{~mL})$ and stirred for $2 \mathrm{~h}$ at $0{ }^{\circ} \mathrm{C}$, then for $16 \mathrm{~h}$ at room temperature. The reaction mixture was then poured into $1 \mathrm{M}$ hydrochloric acid $(150 \mathrm{~mL})$ containing $100 \mathrm{~g}$ of crushed ice and the aqueous layer was extracted with dichloromethane $(3 \times 150 \mathrm{~mL})$. The combined organic layers were washed with $5 \%$ sodium bicarbonate $(1 \times 200 \mathrm{~mL})$ and brine $(1 \times 200 \mathrm{~mL})$ and filtered over celite and magnesium sulfate. The crude product is then purified by recrystallization from ethyl acetate to give the title compound 7 as an off-white solid (33.2 g, 82\%). ${ }^{1} \mathrm{H}$ NMR (400 MHz, Chloroform- $d$ ) $\delta 10.34(\mathrm{~s}, 1 \mathrm{H}), 8.41(\mathrm{~s}, 1 \mathrm{H}), 6.09$ (ddt, $J$ $=17.0,10.4,5.2 \mathrm{~Hz}, 1 \mathrm{H}), 5.49(\mathrm{dd}, J=17.3,1.4 \mathrm{~Hz}, 1 \mathrm{H}), 5.39(\mathrm{dd}, J=10.5,1.3 \mathrm{~Hz}, 1 \mathrm{H}), 4.73(\mathrm{~d}, J=5.3 \mathrm{~Hz}$, 1H), $3.98(\mathrm{~s}, 3 \mathrm{H}), 3.86(\mathrm{~s}, 3 \mathrm{H}) .{ }^{13} \mathrm{C}$ NMR $(151 \mathrm{MHz}$, Chloroform- $d$ ) $\delta 187.48,165.67,165.02,165.00,133.94$, 131.70, 118.80, 118.08, 113.00, 96.04, 69.58, 56.35, 51.92. HRMS (ESI $\left.{ }^{+}\right)$: Calcd for $\mathrm{C}_{13} \mathrm{H}_{14} \mathrm{O}_{5} \mathrm{Na}^{+}\left[\mathrm{M}^{+} \mathrm{Na}^{+}\right.$ 273.0733, found 273.0726 .

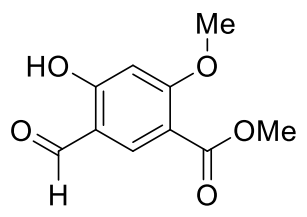

methyl 5-formyl-4-hydroxy-2-methoxybenzoate (1)

To a solution of methyl 5-formyl-4-allyloxy-2-methoxybenzoate 7 (30.0 g, $0.12 \mathrm{~mol})$ in methanol (200 mL) was added potassium carbonate $(49.7 \mathrm{~g}, 0.36 \mathrm{~mol})$ and tetrakis(triphenylphosphine)palladium(0) (1.39 g, $1.2 \mathrm{mmol})$ and brought to reflux while stirring for $3 \mathrm{~h}$. The reaction mixture was then concentrated in vacuo and to the residue was added $1 \mathrm{M}$ aqueous hydrochloric acid $(100 \mathrm{~mL})$ and stirred for $15 \mathrm{~m}$ at $0{ }^{\circ} \mathrm{C}$. The solid is collected by filtration, washed with water, triturated with ethyl acetate, and dried to give the title compound $\mathbf{1}$ as an offwhite powder $(18.9 \mathrm{~g}, 75 \%) .{ }^{1} \mathrm{H}$ NMR $(400 \mathrm{MHz}$, Chloroform- $d) \delta 11.68(\mathrm{~s}, 1 \mathrm{H}), 9.77(\mathrm{~s}, 1 \mathrm{H}), 8.17(\mathrm{~s}, 1 \mathrm{H})$, 6.49 (s, 1H), 3.97 (s, 3H), 3.89 (s, 3H). ${ }^{13} \mathrm{C}$ NMR (101 MHz, Chloroform-d) $\delta$ 194.48, 194.43, 166.87, 166.37, 
164.78, 139.41, 114.33, 112.86, 100.02, 56.61, 52.07. HRMS (ESI $\left.{ }^{+}\right)$Calcd for $\mathrm{C}_{10} \mathrm{H}_{11} \mathrm{O}_{5}{ }^{+}[\mathrm{M}+\mathrm{H}]^{+}$211.0601, found 211.0602 .

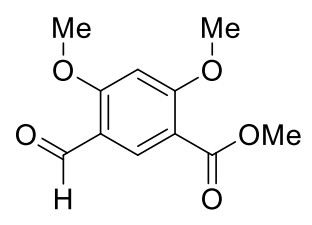

\section{methyl 5-formyl-2,4-dimethoxybenzoate (2a)}

To a stirred solution of methyl 2,4-dimethoxybenzoate $(10.0 \mathrm{~g}, 51 \mathrm{mmol})$ and dichloromethyl methyl ether $(13.8 \mathrm{~mL}, 153 \mathrm{mmol})$ in dichloromethane $(100 \mathrm{~mL})$ at $0{ }^{\circ} \mathrm{C}$ was added dropwise titanium (IV) chloride (16.7 $\mathrm{mL}, 153 \mathrm{mmol})$ in dichloromethane $(20 \mathrm{~mL})$ and stirred for $2 \mathrm{~h}$ at $0{ }^{\circ} \mathrm{C}$, then for $16 \mathrm{~h}$ at room temperature. The reaction mixture was then poured into $1 \mathrm{M}$ hydrochloric acid $(100 \mathrm{~mL})$ containing $50 \mathrm{~g}$ of crushed ice and the aqueous layer was extracted with dichloromethane $(2 \times 100 \mathrm{~mL})$. The combined organic layers were washed with $5 \%$ sodium bicarbonate $(1 \times 100 \mathrm{~mL})$ and brine $(1 \times 100 \mathrm{~mL})$ and filtered over celite and magnesium sulfate. The crude product is then purified by recrystallization from acetonitrile to give the title compound $2 \mathbf{a}$ as a white solid (10.4 g, 91\%). ${ }^{1} \mathrm{H}$ NMR (600 MHz, Chloroform- $d$ ) $\delta 10.29$ (s, 1H), 8.42 (s, 1H), 6.48 (s, $\left.1 \mathrm{H}\right), 4.02$ $(\mathrm{d}, J=2.8 \mathrm{~Hz}, 6 \mathrm{H}), 3.88$ (s, 3H). ${ }^{13} \mathrm{C}$ NMR $(151 \mathrm{MHz}$, Chloroform- $d$ ) $\delta 187.56,165.97,165.87,165.01,134.18$, 117.90, 112.80, 94.85, 56.39, 55.95, 51.91. HRMS (ESI ${ }^{+}$: Calcd for $\mathrm{C}_{11} \mathrm{H}_{13} \mathrm{O}_{5}{ }^{+}[\mathrm{M}+\mathrm{H}]^{+}$225.0757, found 225.0762 .

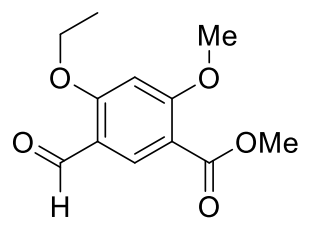

methyl 4-ethoxy-5-formyl-2-methoxybenzoate (2b)

According to general procedure (a): methyl 5-formyl-4-hydroxy-2-methoxybenzoate 1 (1.0 g, $4.8 \mathrm{mmol}$ ), potassium carbonate $(1.3 \mathrm{~g}, 9.5 \mathrm{mmol})$, and iodoethane $(0.57 \mathrm{~mL}, 7.1 \mathrm{mmol})$ gave a crude product that was recrystallized from methylene chloride and hexanes to give the title compound $\mathbf{2 b}$ as a white solid $(0.74 \mathrm{~g}$, 65\%). ${ }^{1} \mathrm{H}$ NMR (400 MHz, Chloroform- $d$ ) $\delta 10.32$ (s, 1H), $8.41(\mathrm{~s}, 1 \mathrm{H}), 6.45$ (s, 1H), 4.22 (q, J = 7.0 Hz, 2H), $3.99(\mathrm{~s}, 3 \mathrm{H}), 3.86(\mathrm{~s}, 3 \mathrm{H}), 1.53(\mathrm{t}, J=6.8 \mathrm{~Hz}, 3 \mathrm{H}) .{ }^{13} \mathrm{C}$ NMR $(101 \mathrm{MHz}$, Chloroform- $d$ ) $\delta 187.65,165.79$, 165.52, 165.06, 133.77, 117.87, 112.68, 95.47, 64.65, 56.33, 51.88, 14.48. HRMS (ESI $\left.{ }^{+}\right)$: Calcd for $\mathrm{C}_{12} \mathrm{H}_{15} \mathrm{O}_{5}{ }^{+}$ $[\mathrm{M}+\mathrm{H}]^{+}$239.0914, found 239.0918.

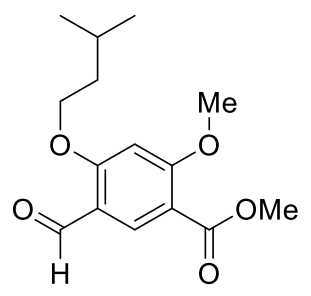

methyl 5-formyl-4-(isopentyloxy)-2-methoxybenzoate (2c)

According to general procedure (a): methyl 5-formyl-4-hydroxy-2-methoxybenzoate 1 (1.0 g, $4.8 \mathrm{mmol})$, potassium carbonate $(1.3 \mathrm{~g}, 9.5 \mathrm{mmol})$, and 1-iodo-3-methylbutane $(0.94 \mathrm{~mL}, 7.1 \mathrm{mmol})$ gave a crude product that was recrystallized from methylene chloride and hexanes to give the title compound $\mathbf{2 c}$ as a white solid (0.97 g, 73\%). ${ }^{1} \mathrm{H}$ NMR (400 MHz, Chloroform- $d$ ) $\delta 10.31(\mathrm{~s}, 1 \mathrm{H}), 8.41(\mathrm{~s}, 1 \mathrm{H}), 6.46(\mathrm{~s}, 1 \mathrm{H}), 4.17(\mathrm{t}, J=6.4 \mathrm{~Hz}, 2 \mathrm{H})$, $3.99(\mathrm{~s}, 3 \mathrm{H}), 3.86(\mathrm{~s}, 3 \mathrm{H}), 1.87(\mathrm{~m}, 1 \mathrm{H}), 1.82-1.75(\mathrm{~m}, 2 \mathrm{H}), 1.00(\mathrm{~d}, J=6.5 \mathrm{~Hz}, 6 \mathrm{H}) .{ }^{13} \mathrm{C} \mathrm{NMR}(101 \mathrm{MHz}$, Chloroform- $d$ ) $\delta 187.58,165.82,165.67,165.05,133.71,117.93,112.65,95.45,67.37,56.34,51.88,37.56$, 25.09, 22.53. HRMS (ESI $\left.{ }^{+}\right)$: Calcd for $\mathrm{C}_{15} \mathrm{H}_{21} \mathrm{O}_{5}{ }^{+}[\mathrm{M}+\mathrm{H}]^{+}$281.1384, found 281.1393. 


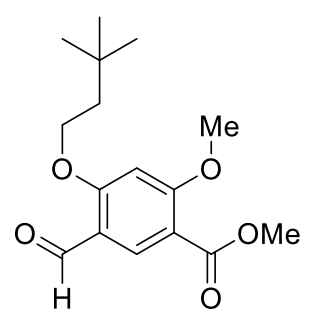

methyl 4-(3,3-dimethylbutoxy)-5-formyl-2-methoxybenzoate (2d)

To a stirred solution of methyl 5-formyl-4-hydroxy-2-methoxybenzoate 1 (0.9 g, $4.3 \mathrm{mmol}$ ), diphenyl-2-

pyridylphosphine $(1.69 \mathrm{~g}, 6.4 \mathrm{mmol})$, and 3,3-dimethylbutanol $(0.62 \mathrm{~mL}, 5.1 \mathrm{mmol})$ in tetrahydrofuran $(20 \mathrm{~mL})$ was added diisopropyl azodicarboxylate $(1.01 \mathrm{~mL}, 5.1 \mathrm{mmol})$ dropwise. The reaction mixture was stirred $4 \mathrm{~h}$ at room temperature and quenched with water. The reaction mixture was concentrated in vacuo and the residue was dissolved in ethyl acetate $(100 \mathrm{~mL})$ and the organic layer was washed with $1 \mathrm{M}$ sodium bisulfate $(2 \mathrm{x} 75$ $\mathrm{mL}), 5 \%$ sodium bicarbonate $(3 \times 75 \mathrm{~mL})$ and brine $(1 \times 75 \mathrm{~mL})$, then filtered over celite and magnesium sulfate and concentrated in vacuo. The residue was purified by flash chromatography (ethyl acetate/hexanes) to give the title compound 2d as a white solid (0.94 g, 75\%). ${ }^{1} \mathrm{H}$ NMR (400 MHz, Chloroform- $d$ ) $\delta 10.30(\mathrm{~s}, 1 \mathrm{H})$, $8.40(\mathrm{~s}, 1 \mathrm{H}), 6.46(\mathrm{~s}, 1 \mathrm{H}), 4.20(\mathrm{t}, J=6.9 \mathrm{~Hz}, 2 \mathrm{H}), 4.00(\mathrm{~s}, 3 \mathrm{H}), 3.86(\mathrm{~s}, 3 \mathrm{H}), 1.83(\mathrm{t}, J=6.9 \mathrm{~Hz}, 2 \mathrm{H}), 1.03(\mathrm{~s}$, 9H). ${ }^{13} \mathrm{C}$ NMR (101 MHz, Chloroform- $d$ ) $\delta$ 187.63, 165.84, 165.57, 165.05, 133.72, 117.90, 112.60, 95.39, 66.39, 56.37, 51.87, 42.02, 29.89, 29.77, 21.96. HRMS (ESI ${ }^{+}$: Calcd for $\mathrm{C}_{16} \mathrm{H}_{23} \mathrm{O}_{5}{ }^{+}[\mathrm{M}+\mathrm{H}]^{+}$295.1540, found 295.1552.

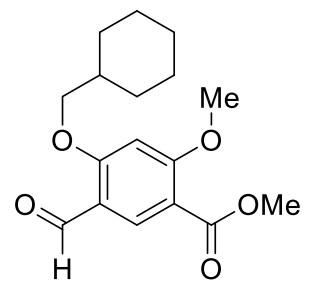

methyl 4-(cyclohexylmethoxy)-5-formyl-2-methoxybenzoate (2e)

According to general procedure (a): methyl 5-formyl-4-hydroxy-2-methoxybenzoate 1 (1.0 g, $4.8 \mathrm{mmol})$, potassium carbonate $(1.3 \mathrm{~g}, 9.5 \mathrm{mmol})$, and cyclohexylmethylbromide $(1.0 \mathrm{~mL}, 7.1 \mathrm{mmol})$ gave a crude product that was recrystallized from methylene chloride and hexanes to give the title compound $\mathbf{2 e}$ as a white solid (1.0 g, 68\%). ${ }^{1} \mathrm{H}$ NMR (400 MHz, Chloroform- $d$ ) $\delta 10.34$ (s, 1H), 8.41 (s, 1H), $6.44(\mathrm{~s}, 1 \mathrm{H}), 3.99$ (s, $\left.3 \mathrm{H}\right), 3.92(\mathrm{~d}, J$ $=5.8 \mathrm{~Hz}, 2 \mathrm{H}), 3.86(\mathrm{~s}, 3 \mathrm{H}), 1.98-1.69(\mathrm{~m}, 6 \mathrm{H}), 1.41-1.06(\mathrm{~m}, 5 \mathrm{H}) .{ }^{13} \mathrm{C}$ NMR $(101 \mathrm{MHz}$, Chloroform- $d) \delta$ 187.52, 165.87, 165.84, 165.07, 133.69, 117.98, 112.58, 95.50, 74.18, 56.37, 51.87, 37.63, 29.81, 26.34, 25.71. HRMS (ESI ${ }^{+}$: Calcd for $\mathrm{C}_{17} \mathrm{H}_{23} \mathrm{O}_{5}^{+}[\mathrm{M}+\mathrm{H}]^{+}$307.1540, found 307.1552.

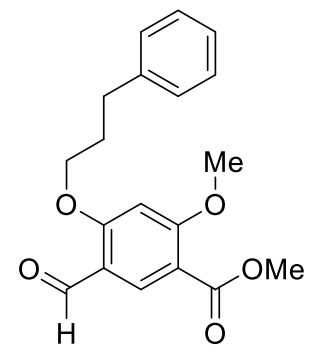

methyl 5-formyl-2-methoxy-4-(3-phenylpropoxy)benzoate (2f)

According to general procedure (a): methyl 5-formyl-4-hydroxy-2-methoxybenzoate 1 (1.0 g, $4.8 \mathrm{mmol})$, potassium carbonate $(1.3 \mathrm{~g}, 9.5 \mathrm{mmol})$, and (3-iodopropyl)benzene $(1.2 \mathrm{~mL}, 7.1 \mathrm{mmol})$ gave a crude product that was recrystallized from methylene chloride and hexanes to give the title compound $\mathbf{2} \mathbf{f}$ as a white solid (1.5 g, 96\%). ${ }^{1} \mathrm{H}$ NMR (400 MHz, Chloroform- $d$ ) $\delta 10.30$ (s, 1H), $8.41(\mathrm{~s}, 1 \mathrm{H}), 7.35-7.27$ (m, 2H), $7.25-7.17$ (m, 
$3 \mathrm{H}), 6.38(\mathrm{~s}, 1 \mathrm{H}), 4.14(\mathrm{t}, J=6.2 \mathrm{~Hz}, 2 \mathrm{H}), 3.93(\mathrm{~s}, 3 \mathrm{H}), 3.86(\mathrm{~s}, 3 \mathrm{H}), 2.86(\mathrm{t}, J=7.4 \mathrm{~Hz}, 2 \mathrm{H}), 2.28-2.16(\mathrm{~m}$, $2 \mathrm{H}) .{ }^{13} \mathrm{C}$ NMR $(101 \mathrm{MHz}$, Chloroform- $d) \delta 187.46,165.78,165.47,165.03,140.73,133.83,128.61,128.48$, 126.27, 117.93, 112.79, 95.47, 67.73, 56.33, 51.90, 32.04, 30.37. HRMS (ESI $)$ : Calcd for $\mathrm{C}_{19} \mathrm{H}_{21} \mathrm{O}_{5}{ }^{+}\left[\mathrm{M}_{+} \mathrm{H}^{+}\right.$ 329.1384 , found 329.1380 .

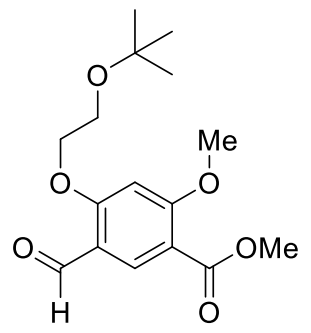

methyl 4-(2-(tert-butoxy)ethoxy)-5-formyl-2-methoxybenzoate (2g)

To a stirred solution of methyl 5-formyl-4-hydroxy-2-methoxybenzoate 1 (2.0 g, $9.5 \mathrm{mmol})$, diphenyl-2pyridylphosphine (3.8 g, $14 \mathrm{mmol})$, and 2-(tert-butoxy)ethan-1-ol (1.6 mL, $11 \mathrm{mmol}$ ) in tetrahydrofuran (30 $\mathrm{mL}$ ) was added diisopropyl azodicarboxylate $(2.3 \mathrm{~mL}, 11 \mathrm{mmol})$ dropwise. The reaction mixture was stirred $4 \mathrm{~h}$ at room temperature and quenched with water. The reaction mixture was concentrated in vacuo and the residue was dissolved in ethyl acetate $(100 \mathrm{~mL})$ and the organic layer was washed with $1 \mathrm{M}$ sodium bisulfate $(2 \mathrm{x} 75$ $\mathrm{mL}), 5 \%$ sodium bicarbonate $(3 \times 75 \mathrm{~mL})$ and brine $(1 \times 75 \mathrm{~mL})$, then filtered over celite and magnesium sulfate and concentrated in vacuo. The residue was purified by flash chromatography (ethyl acetate/hexanes) to give the title compound $2 \mathrm{~g}$ as a white solid (1.86 g, 63\%). ${ }^{1} \mathrm{H}$ NMR (400 MHz, Chloroform- $d$ ) $\delta 10.32(\mathrm{~s}, 1 \mathrm{H})$, $8.41(\mathrm{~s}, 1 \mathrm{H}), 6.55(\mathrm{~s}, 1 \mathrm{H}), 4.27(\mathrm{t}, J=5.1 \mathrm{~Hz}, 2 \mathrm{H}), 3.98(\mathrm{~s}, 3 \mathrm{H}), 3.86(\mathrm{~s}, 3 \mathrm{H}), 3.80(\mathrm{t}, J=5.0 \mathrm{~Hz}, 2 \mathrm{H}), 1.24(\mathrm{~s}$, 9H). ${ }^{13} \mathrm{C}$ NMR (101 MHz, Chloroform- $d$ ) $\delta$ 187.65, 165.75, 165.72, 165.05, 133.61, 118.04, 112.91, 96.22, 73.65, 69.01, 60.28, 56.38, 51.89, 27.47. HRMS (ESI ${ }^{+}$: Calcd for $\mathrm{C}_{16} \mathrm{H}_{23} \mathrm{O}_{6}{ }^{+}[\mathrm{M}+\mathrm{H}]^{+} 311.1489$, found 311.1480 .

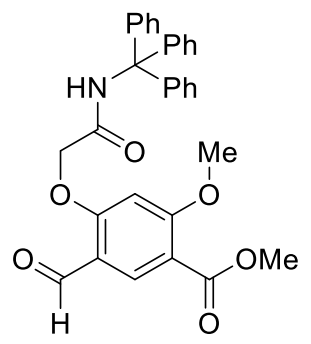

methyl 5-formyl-2-methoxy-4-(2-oxo-2-(tritylamino)ethoxy)benzoate (2h)

According to general procedure (a): methyl 5-formyl-4-hydroxy-2-methoxybenzoate 1 (1.0 g, $4.8 \mathrm{mmol})$, potassium carbonate $(1.3 \mathrm{~g}, 9.5 \mathrm{mmol})$, and 2-bromo- $N$-tritylacetamide $(2.7 \mathrm{~g}, 7.1 \mathrm{mmol})$ gave a crude product that was recrystallized from methylene chloride and hexanes to give the title compound $\mathbf{2} \mathbf{h}$ as a white solid (2.3 g, 95\%). ${ }^{1} \mathrm{H}$ NMR (400 MHz, Chloroform- $d$ ) $\delta 10.06$ (s, 1H), 8.34 (s, 1H), $8.19(\mathrm{~s}, 1 \mathrm{H}), 7.35-7.19(\mathrm{~m}, 15 \mathrm{H})$, $6.41(\mathrm{~s}, 1 \mathrm{H}), 4.64(\mathrm{~s}, 2 \mathrm{H}), 3.92(\mathrm{~s}, 3 \mathrm{H}), 3.89$ (s, 3H). ${ }^{13} \mathrm{C}$ NMR (101 MHz, Chloroform- $\left.d\right) \delta$ 187.08, 165.59, 165.50, 164.71, 162.07, 144.17, 138.23, 128.66, 128.52, 128.12, 128.04, 128.02, 127.30, 127.26, 117.94, 113.80, 96.33, 70.66, 68.00, 56.59, 53.43, 52.14. HRMS (ESI $)$ : Calcd for $\mathrm{C}_{31} \mathrm{H}_{27} \mathrm{O}_{6} \mathrm{Na}^{+}[\mathrm{M}+\mathrm{Na}]^{+} 532.1731$, found 532.1732 .<smiles>[X]Oc1cc(OC)c(C(=O)O)cc1C=O</smiles> 
According to general procedure (b): saponification of methyl 5-formyl-2,4-dimethoxybenzoate $2 \mathbf{a}$ (4.0 g, 18 mmol) gave the title compound 3a as a white solid $(3.7 \mathrm{~g}, 98 \%) .{ }^{1} \mathrm{H}$ NMR (400 MHz, DMSO- $\left.d_{6}\right) \delta 12.58(\mathrm{~s}$, $1 \mathrm{H}), 10.16(\mathrm{~s}, 1 \mathrm{H}), 8.11(\mathrm{~s}, 1 \mathrm{H}), 6.79(\mathrm{~s}, 1 \mathrm{H}), 4.02(\mathrm{~s}, 3 \mathrm{H}), 3.97(\mathrm{~s}, 3 \mathrm{H}) .{ }^{13} \mathrm{C} \mathrm{NMR}\left(101 \mathrm{MHz}, \mathrm{DMSO}-d_{6}\right) \delta$ 186.98, 165.68, 165.59, 165.43, 132.54, 116.92, 113.16, 96.65, 56.53, 56.50. HRMS (ESI $\left.{ }^{+}\right)$: Calcd for $\mathrm{C}_{10} \mathrm{H}_{10} \mathrm{O}_{5} \mathrm{Na}^{+}[\mathrm{M}+\mathrm{Na}]^{+} 233.0420$, found 233.0421.

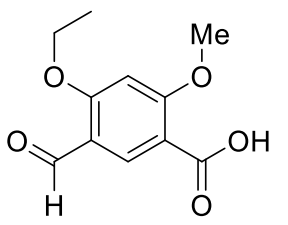

\section{4-ethoxy-5-formyl-2-methoxybenzoic acid (3b)}

According to general procedure (b): saponification of methyl 4-ethoxy-5-formyl-2-methoxybenzoate $2 \mathbf{b}(0.70 \mathrm{~g}$, $2.9 \mathrm{mmol})$ gave the title compound $\mathbf{3 b}$ as a white solid $(0.55 \mathrm{~g}, 83 \%) .{ }^{1} \mathrm{H}$ NMR $(400 \mathrm{MHz}$, Chloroform- $d) \delta$ $10.33(\mathrm{~s}, 1 \mathrm{H}), 8.66(\mathrm{~s}, 1 \mathrm{H}), 6.51(\mathrm{~s}, 1 \mathrm{H}), 4.25(\mathrm{q}, J=7.0 \mathrm{~Hz}, 2 \mathrm{H}), 4.13(\mathrm{~s}, 3 \mathrm{H}), 1.55(\mathrm{t}, J=7.0 \mathrm{~Hz}, 3 \mathrm{H}) .{ }^{13} \mathrm{C}$ NMR (101 MHz, Chloroform- $d$ ) $\delta 187.29,165.92,164.31,163.85,135.99,119.56,110.69,95.37,65.06,56.99$, 14.44. HRMS (ESI ${ }^{+}$: Calcd for $\mathrm{C}_{11} \mathrm{H}_{12} \mathrm{O}_{5} \mathrm{Na}^{+}[\mathrm{M}+\mathrm{Na}]^{+}$247.0577, found 247.0578.

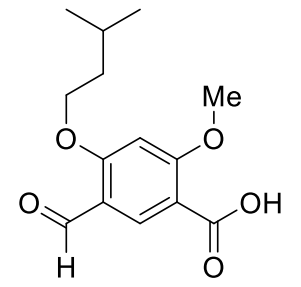

\section{5-formyl-4-(isopentyloxy)-2-methoxybenzoic acid (3c)}

According to general procedure (b): saponification of methyl 4-(isopentyloxy)-5-formyl-2-methoxybenzoate 2c $(0.80 \mathrm{~g}, 2.9 \mathrm{mmol})$ gave the title compound $3 \mathrm{c}$ as a white solid $(0.73 \mathrm{~g}, 96 \%) .{ }^{1} \mathrm{H}$ NMR (400 MHz, Chloroformd) $\delta 10.32(\mathrm{~s}, 1 \mathrm{H}), 8.65(\mathrm{~s}, 1 \mathrm{H}), 6.52(\mathrm{~s}, 1 \mathrm{H}), 4.19(\mathrm{t}, J=6.4 \mathrm{~Hz}, 2 \mathrm{H}), 4.13(\mathrm{~s}, 3 \mathrm{H}), 1.88(\mathrm{~m}, 1 \mathrm{H}), 1.80(\mathrm{~m}, 2 \mathrm{H})$, $1.01(\mathrm{~d}, J=6.5 \mathrm{~Hz}, 6 \mathrm{H}) .{ }^{13} \mathrm{C}$ NMR $(101 \mathrm{MHz}$, Chloroform- $d$ ) $\delta 187.21,166.08,164.44,163.92,135.90,119.61$, 110.68, 95.38, 67.77, 57.00, 37.49, 25.07, 22.51. HRMS (ESI $\left.{ }^{+}\right)$: Calcd for $\mathrm{C}_{14} \mathrm{H}_{18} \mathrm{O}_{5} \mathrm{Na}^{+}[\mathrm{M}+\mathrm{Na}]^{+} 289.1046$, found 289.1045 .

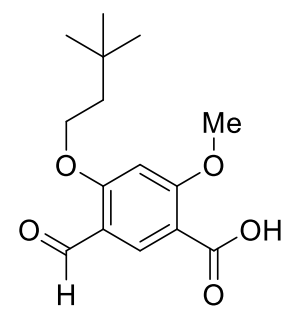

\section{4-(3,3-dimethylbutoxy)-5-formyl-2-methoxybenzoic acid (3d)}

According to general procedure (b): saponification of methyl 4-(3,3-dimethylbutoxy)-5-formyl-2methoxybenzoate $2 \mathrm{~d}(0.74 \mathrm{~g}, 2.5 \mathrm{mmol})$ gave the title compound 3d as a white solid $(0.68 \mathrm{~g}, 96 \%) .{ }^{1} \mathrm{H} \mathrm{NMR}$ $(400 \mathrm{MHz}$, Chloroform- $d) \delta 10.31(\mathrm{~s}, 1 \mathrm{H}), 8.65(\mathrm{~s}, 1 \mathrm{H}), 6.52(\mathrm{~s}, 1 \mathrm{H}), 4.23(\mathrm{t}, J=6.9 \mathrm{~Hz}, 2 \mathrm{H}), 4.14(\mathrm{~s}, 3 \mathrm{H}), 1.85$ $(\mathrm{t}, J=6.9 \mathrm{~Hz}, 2 \mathrm{H}), 1.04(\mathrm{~s}, 9 \mathrm{H}) .{ }^{13} \mathrm{C}$ NMR $(101 \mathrm{MHz}$, Chloroform- $d) \delta 187.27,165.98,164.64,164.01,135.88$, 119.53, 110.67, 95.31, 66.80, 57.00, 41.96, 29.90, 29.75. HRMS (ESI ${ }^{+}$: Calcd for $\mathrm{C}_{15} \mathrm{H}_{20} \mathrm{O}_{5} \mathrm{Na}^{+}[\mathrm{M}+\mathrm{Na}]^{+}$ 303.1203, found 303.1206. 


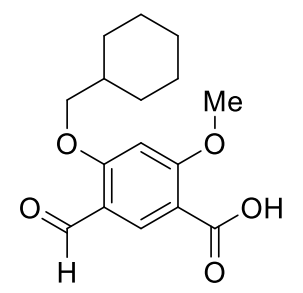

4-(cyclohexylmethoxy)-5-formyl-2-methoxybenzoic acid (3e)

According to general procedure (b): saponification of methyl 4-(cyclohexylmethoxy)-5-formyl-2-

methoxybenzoate $\mathbf{2 e}(0.90 \mathrm{~g}, 2.9 \mathrm{mmol})$ gave the title compound $\mathbf{3 e}$ as a white solid $(0.78 \mathrm{~g}, 91 \%) .{ }^{1} \mathrm{H} \mathrm{NMR}$ $(400 \mathrm{MHz}$, Chloroform- $d) \delta 10.35(\mathrm{~s}, 1 \mathrm{H}), 8.65(\mathrm{~s}, 1 \mathrm{H}), 6.50(\mathrm{~s}, 1 \mathrm{H}), 4.13(\mathrm{~s}, 3 \mathrm{H}), 3.94(\mathrm{~d}, J=5.9 \mathrm{~Hz}, 2 \mathrm{H}), 2.00$ $-1.69(\mathrm{~m}, 6 \mathrm{H}), 1.42-1.06(\mathrm{~m}, 5 \mathrm{H}) .{ }^{13} \mathrm{C}$ NMR (101 MHz, Chloroform-d) $\delta 187.16,166.27,164.42,163.91$, 135.90, 119.67, 110.60, 95.42, 74.56, 57.02, 37.58, 29.78, 26.30, 25.67. Calcd for $\mathrm{C}_{16} \mathrm{H}_{20} \mathrm{O}_{5} \mathrm{Na}^{+}[\mathrm{M}+\mathrm{Na}]^{+}$ 315.1203, found 315.1201 .

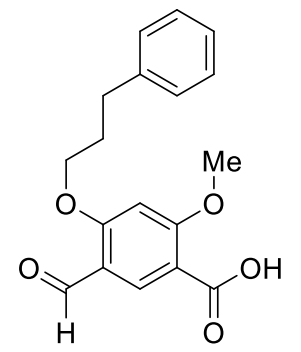

\section{5-formyl-4-(3-phenylpropoxy)-2-methoxybenzoic acid (3f)}

According to general procedure (b): saponification of methyl 5-formyl-2-methoxy-4-(3-

phenylpropoxy)benzoate $2 \mathbf{f}(1.4 \mathrm{~g}, 4.3 \mathrm{mmol})$ gave the title compound $\mathbf{3 f}$ as a white solid $(1.2 \mathrm{~g}, 89 \%) .{ }^{1} \mathrm{H}$ NMR (400 MHz, Chloroform- $d) \delta 10.30(\mathrm{~s}, 1 \mathrm{H}), 8.64(\mathrm{~s}, 1 \mathrm{H}), 7.31-7.17(\mathrm{~m}, \mathrm{H}), 6.43(\mathrm{~s}, 1 \mathrm{H}), 4.16(\mathrm{t}, J=6.2$ $\mathrm{Hz}, 2 \mathrm{H}), 4.06(\mathrm{~s}, 3 \mathrm{H}), 2.87(\mathrm{t}, J=7.4 \mathrm{~Hz}, 2 \mathrm{H}), 2.24(\mathrm{~m}, 2 \mathrm{H}) .{ }^{13} \mathrm{C}$ NMR $(101 \mathrm{MHz}$, Chloroform-d) $\delta 187.13$, 165.88, 164.75, 164.02, 140.61, 135.98, 128.64, 128.49, 126.32, 119.50, 110.81, 95.42, 68.09, 56.95, 31.96, 30.25. HRMS (ESI ${ }^{+}$): Calcd for $\mathrm{C}_{18} \mathrm{H}_{18} \mathrm{O}_{5} \mathrm{Na}^{+}[\mathrm{M}+\mathrm{Na}]^{+} 337.1046$, found 337.1047

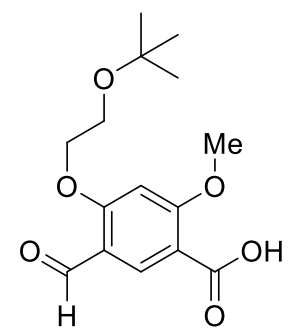

\section{5-formyl-4-(2-(tert-butoxy)ethoxy)-2-methoxybenzoic acid (3g)}

According to general procedure (b): saponification of methyl 4-(2-(tert-butoxy)ethoxy)-5-formyl-2methoxybenzoate $\mathbf{2 g}(1.8 \mathrm{~g}, 5.8 \mathrm{mmol})$ gave the title compound $\mathbf{3 g}$ as a white solid $(1.68 \mathrm{~g}, 98 \%)$. ${ }^{1} \mathrm{H} \mathrm{NMR}$ $(400 \mathrm{MHz}$, Chloroform- $d) \delta 10.33(\mathrm{~s}, 1 \mathrm{H}), 8.62(\mathrm{~s}, 1 \mathrm{H}), 6.64(\mathrm{~s}, 1 \mathrm{H}), 4.31(\mathrm{t}, J=5.0 \mathrm{~Hz}, 2 \mathrm{H}), 4.10(\mathrm{~s}, 3 \mathrm{H}), 3.82$ $(\mathrm{t}, J=5.0 \mathrm{~Hz}, 2 \mathrm{H}), 1.24(\mathrm{~s}, 9 \mathrm{H}) .{ }^{13} \mathrm{C}$ NMR $(101 \mathrm{MHz}$, Chloroform- $d) \delta 187.32,166.24,164.78,163.98,135.66$, 119.56, 110.99, 96.33, 73.79, 69.39, 60.33, 56.99, 27.47, 21.95. HRMS (ESI ${ }^{+}$: Calcd for $\mathrm{C}_{15} \mathrm{H}_{20} \mathrm{O}_{6} \mathrm{Na}^{+}$ $[\mathrm{M}+\mathrm{Na}]^{+} 319.1152$, found 319.1152 . 


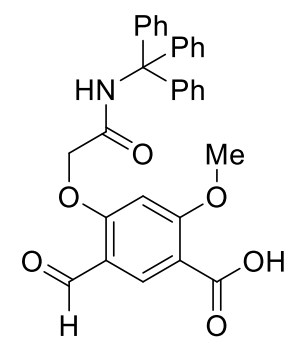

5-formyl-4-(2-oxo-2-(tritylamino)ethoxy)-2-methoxybenzoic acid (3h)

According to general procedure (b): saponification of methyl 5-formyl-2-methoxy-4-(2-oxo-2-

(tritylamino)ethoxy)benzoate $\mathbf{2 g}(2.4 \mathrm{~g}, 4.7 \mathrm{mmol})$ gave the title compound $\mathbf{3 g}$ as a white solid $(1.89 \mathrm{~g}, 79 \%) .{ }^{1} \mathrm{H}$ NMR (400 MHz, DMSO-d $\left.d_{6}\right) \delta 12.63(\mathrm{~s}, 1 \mathrm{H}), 10.14(\mathrm{~s}, 1 \mathrm{H}), 8.82(\mathrm{~s}, 1 \mathrm{H}), 8.11(\mathrm{~s}, 1 \mathrm{H}), 7.34-7.13(\mathrm{~m}, 15 \mathrm{H})$, $6.69(\mathrm{~s}, 1 \mathrm{H}), 5.01(\mathrm{~s}, 2 \mathrm{H}), 3.88(\mathrm{~s}, 3 \mathrm{H}) .{ }^{13} \mathrm{C}$ NMR (101 MHz, DMSO- $\left.d_{6}\right) \delta 187.26,166.13,165.51,164.87$, 163.77, 144.34, 133.20, 128.31, 128.18, 127.71, 127.53, 126.55, 117.06, 113.60, 97.54, 69.31, 67.44, 66.91, 56.40, 54.81, 25.02. HRMS (ESI ${ }^{+}$): Calcd for $\mathrm{C}_{30} \mathrm{H}_{25} \mathrm{NO}_{6} \mathrm{Na}^{+}[\mathrm{M}+\mathrm{Na}]^{+}$518.1574, found 518.1562.

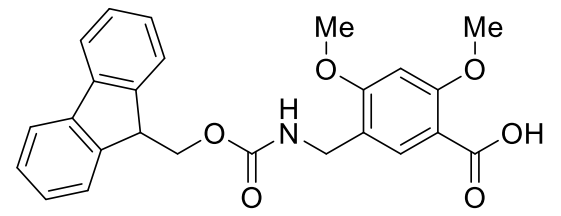

5-(Fmoc-aminomethyl)-2,4-dimethoxybenzoic acid (4a, Fmoc-Mmb1-OH)

According to general procedure (c): To a solution of 5-formyl-2,4-dimethoxybenzoic acid 3a (3.5 g, $17 \mathrm{mmol})$ and 9-fluorenylmethyl carbamate $(4.0 \mathrm{~g}, 17 \mathrm{mmol})$ in acetonitrile $(30 \mathrm{~mL})$ was added trifluoroacetic acid $(2.6$ $\mathrm{mL}, 33 \mathrm{mmol})$ and triethylsilane $(8.0 \mathrm{~mL}, 50 \mathrm{mmol})$ and stirred at $50{ }^{\circ} \mathrm{C}$ until complete $(6 \mathrm{~h}$, monitored by TLC). The reaction mixture was then concentrated in vacuo and the residue was purified by flash chromatography (ethyl acetate/hexanes) to give the title compound $\mathbf{4 a}$ as a white solid (6.9 g, 96\%). ${ }^{1} \mathrm{H} \mathrm{NMR}$ $\left(600 \mathrm{MHz}, \mathrm{DMSO}-d_{6}\right) \delta 7.90(\mathrm{~d}, J=7.5 \mathrm{~Hz}, 2 \mathrm{H}), 7.76(\mathrm{t}, J=6.1 \mathrm{~Hz}, 1 \mathrm{H}), 7.72(\mathrm{~d}, J=7.5 \mathrm{~Hz}, 2 \mathrm{H}), 7.66(\mathrm{~s}, 1 \mathrm{H})$, $7.42(\mathrm{t}, J=7.4 \mathrm{~Hz}, 2 \mathrm{H}), 7.33(\mathrm{t}, J=7.3 \mathrm{~Hz}, 2 \mathrm{H}), 6.68(\mathrm{~s}, 1 \mathrm{H}), 4.32-4.20(\mathrm{~m}, 3 \mathrm{H}), 4.12(\mathrm{~d}, J=5.9 \mathrm{~Hz}, 2 \mathrm{H})$, $3.90(\mathrm{~s}, 3 \mathrm{H}), 3.87(\mathrm{~s}, 6 \mathrm{H}) .{ }^{13} \mathrm{C}$ NMR $\left(151 \mathrm{MHz}, \mathrm{DMSO}-d_{6}\right) \delta 167.02,161.19,160.55,156.71,144.35,141.17$, 131.50, 128.09, 128.07, 127.56, 127.53, 125.72, 125.62, 120.58, 119.20, 111.76, 96.61, 66.05, 56.57, 56.33, 47.14, 38.62. HRMS (ESI ${ }^{+}$): Calcd for $\mathrm{C}_{25} \mathrm{H}_{23} \mathrm{NO}_{6} \mathrm{Na}^{+}[\mathrm{M}+\mathrm{Na}]^{+} 456.1418$, found 456.1420.

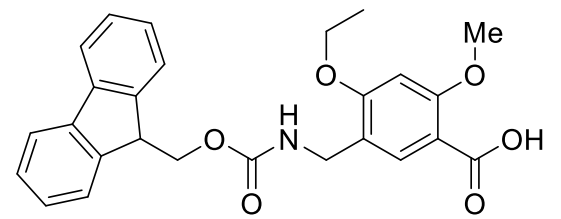

\section{5-(Fmoc-aminomethyl)-4-ethoxy-2-methoxybenzoic acid (4b, Fmoc-Mmb2-OH)}

According to general procedure (c): 5-formyl-4-ethoxy-2-methoxybenzoic acid 3b (0.5 g, $2.2 \mathrm{mmol})$ gave a crude product that was purified by flash chromatography (ethyl acetate/hexanes) to give the title compound $\mathbf{4 b}$ as a white powder $(0.85 \mathrm{~g}, 85 \%) .{ }^{1} \mathrm{H}$ NMR $\left(400 \mathrm{MHz}, \mathrm{DMSO}-d_{6}\right) \delta 12.33(\mathrm{~s}, 1 \mathrm{H}), 7.89(\mathrm{~d}, J=7.5 \mathrm{~Hz}, 2 \mathrm{H}), 7.71$ $(\mathrm{d}, J=7.2 \mathrm{~Hz}, 3 \mathrm{H}), 7.65(\mathrm{~s}, 1 \mathrm{H}), 7.41(\mathrm{t}, J=7.4 \mathrm{~Hz}, 2 \mathrm{H}), 7.33(\mathrm{t}, J=7.4 \mathrm{~Hz}, 2 \mathrm{H}), 6.66(\mathrm{~s}, 1 \mathrm{H}), 4.32-4.09$ (m, $7 \mathrm{H}), 3.84(\mathrm{~s}, 3 \mathrm{H}), 1.36(\mathrm{t}, J=6.9 \mathrm{~Hz}, 3 \mathrm{H}) .{ }^{13} \mathrm{C}$ NMR $\left(101 \mathrm{MHz}, \mathrm{DMSO}-d_{6}\right) \delta 167.04,160.51,160.46,156.72$, 144.36, 141.17, 131.46, 128.09, 127.56, 125.72, 120.59, 119.34, 111.70, 97.28, 66.05, 64.32, 56.56, 47.15, 38.67, 14.95. HRMS (ESI ${ }^{+}$): Calcd for $\mathrm{C}_{26} \mathrm{H}_{25} \mathrm{NO}_{6} \mathrm{Na}^{+}[\mathrm{M}+\mathrm{Na}]^{+} 470.1574$, found 470.1575 . 


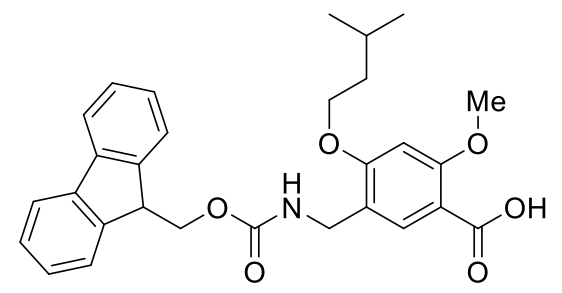

5-(Fmoc-aminomethyl)-4-(isopentyloxy)-2-methoxybenzoic acid (4c, Fmoc-Mmb3-OH)

According to general procedure (c): 5-formyl-4-(isopentyloxy)-2-methoxybenzoic acid 3c $(0.7 \mathrm{~g}, 2.6 \mathrm{mmol})$ gave a crude product that was purified by flash chromatography (ethyl acetate/hexanes) to give the title compound $4 \mathbf{c}$ as a white powder $(0.94 \mathrm{~g}, 73 \%) .{ }^{1} \mathrm{H}$ NMR $\left(400 \mathrm{MHz}, \mathrm{DMSO}-d_{6}\right) \delta 12.11(\mathrm{~s}, 1 \mathrm{H}), 7.89(\mathrm{~d}, J=7.5$ $\mathrm{Hz}, 2 \mathrm{H}), 7.74-7.65(\mathrm{~m}, 3 \mathrm{H}), 7.64(\mathrm{~s}, 1 \mathrm{H}), 7.41(\mathrm{t}, J=7.4 \mathrm{~Hz}, 2 \mathrm{H}), 7.33(\mathrm{t}, J=7.4 \mathrm{~Hz}, 2 \mathrm{H}), 6.68(\mathrm{~s}, 1 \mathrm{H}), 4.32-$ $4.19(\mathrm{~m}, 3 \mathrm{H}), 4.19-4.07(\mathrm{~m}, 4 \mathrm{H}), 3.85(\mathrm{~s}, 3 \mathrm{H}), 1.81(\mathrm{dt}, J=13.3,6.7 \mathrm{~Hz}, 1 \mathrm{H}), 1.65(\mathrm{q}, J=6.6 \mathrm{~Hz}, 2 \mathrm{H}), 0.93$ $(\mathrm{d}, J=6.6 \mathrm{~Hz}, 6 \mathrm{H}) .{ }^{13} \mathrm{C}$ NMR $\left(101 \mathrm{MHz}\right.$, DMSO- $\left.d_{6}\right) \delta 166.45,160.07,159.93,156.10,143.77,140.59,130.82$, $127.51,126.97,125.12,120.01,118.66,111.02$, 96.66, 66.38, 65.49, 56.00, 46.57, 38.15, 37.20, 24.47, 22.33. HRMS (ESI ${ }^{+}$): Calcd for $\mathrm{C}_{29} \mathrm{H}_{31} \mathrm{NO}_{6} \mathrm{Na}^{+}[\mathrm{M}+\mathrm{Na}]^{+}$512.2044, found 512.2044.

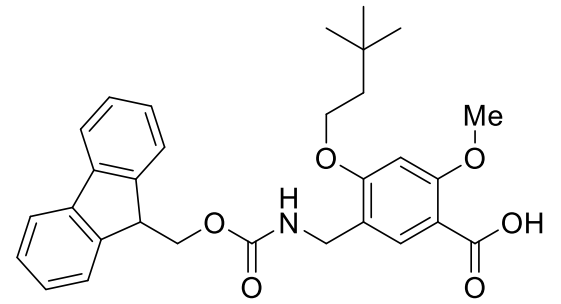

\section{5-(Fmoc-aminomethyl)-4-(3,3-dimethylbutoxy)-2-methoxybenzoic acid (4d, Fmoc-Mmb4-OH)}

According to general procedure (c): 5-formyl-4-(3,3-dimethylbutoxy)-2-methoxybenzoic acid 3d (0.6 g, 2.1 $\mathrm{mmol}$ ) gave a crude product that was purified by flash chromatography (ethyl acetate/hexanes) to give the title compound 4d as a white powder $(0.80 \mathrm{~g}, 74 \%) .{ }^{1} \mathrm{H}$ NMR $\left(400 \mathrm{MHz}, \mathrm{DMSO}-d_{6}\right) \delta 12.11(\mathrm{~s}, 1 \mathrm{H}), 7.89(\mathrm{~d}, J=7.5$ $\mathrm{Hz}, 2 \mathrm{H}), 7.71(\mathrm{~d}, J=7.4 \mathrm{~Hz}, 3 \mathrm{H}), 7.64(\mathrm{~s}, 1 \mathrm{H}), 7.41(\mathrm{t}, J=7.4 \mathrm{~Hz}, 2 \mathrm{H}), 7.33(\mathrm{t}, J=7.4 \mathrm{~Hz}, 2 \mathrm{H}), 6.69(\mathrm{~s}, 1 \mathrm{H})$, $4.32-4.20(\mathrm{~m}, 3 \mathrm{H}), 4.20-4.08(\mathrm{~m}, 4 \mathrm{H}), 3.86(\mathrm{~s}, 3 \mathrm{H}), 1.70(\mathrm{t}, J=6.9 \mathrm{~Hz}, 2 \mathrm{H}), 0.97(\mathrm{~s}, 9 \mathrm{H}) .{ }^{13} \mathrm{C}$ NMR $(101$ MHz, DMSO- $\left.d_{6}\right) \delta 168.23,167.04,160.61,160.52,156.69,144.35,141.17,137.18,131.30,128.09,127.56$, 125.71, 120.58, 119.21, 111.57, 97.19, 66.06, 56.62, 47.15, 42.27, 38.77, 30.03. HRMS (ESI ${ }^{+}$): Calcd for $\mathrm{C}_{30} \mathrm{H}_{33} \mathrm{NO}_{6} \mathrm{Na}^{+}[\mathrm{M}+\mathrm{Na}]^{+}$526.2200, found 526.2198.

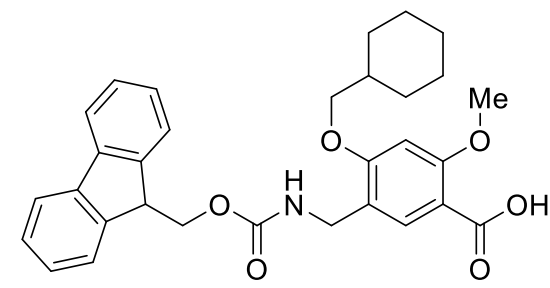

\section{5-(Fmoc-aminomethyl)-4-(cyclohexylmethoxy)-2-methoxybenzoic acid (4e, Fmoc-Mmb5-OH)}

According to general procedure (c): 5-formyl-4-(cyclohexylmethoxy)-2-methoxybenzoic acid 3e (0.6 g, 2.1 $\mathrm{mmol}$ ) gave a crude product that was purified by flash chromatography (ethyl acetate/hexanes) to give the title compound $4 \mathbf{e}$ as a white powder $(0.79 \mathrm{~g}, 74 \%) .{ }^{1} \mathrm{H}$ NMR $\left(400 \mathrm{MHz}, \mathrm{DMSO}-d_{6}\right) \delta 12.11(\mathrm{~s}, 1 \mathrm{H}), 7.89(\mathrm{~d}, J=7.5$ $\mathrm{Hz}, 2 \mathrm{H}), 7.74-7.66(\mathrm{~m}, 3 \mathrm{H}), 7.64(\mathrm{~s}, 1 \mathrm{H}), 7.41$ (t, $J=7.4 \mathrm{~Hz}, 2 \mathrm{H}), 7.33$ (t, $J=7.4 \mathrm{~Hz}, 2 \mathrm{H}), 6.64(\mathrm{~s}, 1 \mathrm{H}), 4.32-$ $4.19(\mathrm{~m}, 3 \mathrm{H}), 4.14(\mathrm{~d}, J=5.9 \mathrm{~Hz}, 2 \mathrm{H}), 3.90(\mathrm{~d}, J=5.9 \mathrm{~Hz}, 2 \mathrm{H}), 3.84(\mathrm{~s}, 3 \mathrm{H}), 1.88-1.57(\mathrm{~m}, 6 \mathrm{H}), 1.33-1.00$ $(\mathrm{m}, 5 \mathrm{H}) .{ }^{13} \mathrm{C}$ NMR $\left(101 \mathrm{MHz}\right.$, DMSO- $\left.d_{6}\right) \delta 167.02,160.76,160.55,156.66,144.36,141.17,131.49,128.09$, $127.55,125.70,120.59,119.22,111.54,97.15,73.58,66.09,56.58,47.15,38.79,37.62,29.66,26.46,25.82$. HRMS (ESI ${ }^{+}$): Calcd for $\mathrm{C}_{31} \mathrm{H}_{33} \mathrm{NO}_{6} \mathrm{Na}^{+}[\mathrm{M}+\mathrm{Na}]^{+}$538.2200, found 538.2197. 


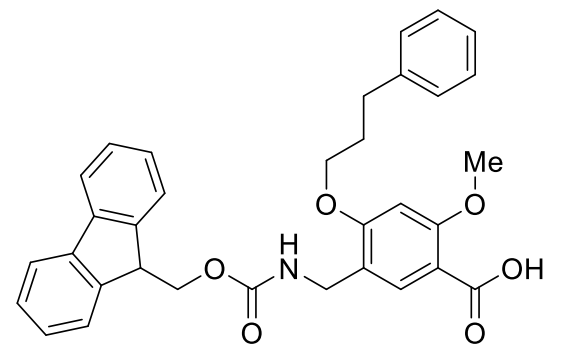

5-(Fmoc-aminomethyl)-4-(3-phenylpropoxy)-2-methoxybenzoic acid (4f, Fmoc-Mmb6-OH)

According to general procedure (c): 5-formyl-4-(3-phenylpropoxy)-2-methoxybenzoic acid 3f (1.0 g, $3.2 \mathrm{mmol})$ gave a crude product that was purified by flash chromatography (ethyl acetate/hexanes) to give the title compound $4 \mathbf{f}$ as a white powder $(1.3 \mathrm{~g}, 78 \%)$. ${ }^{1} \mathrm{H}$ NMR $\left(400 \mathrm{MHz}, \mathrm{DMSO}-d_{6}\right) \delta 12.14(\mathrm{~s}, 1 \mathrm{H}), 7.88(\mathrm{~d}, J=7.6$ $\mathrm{Hz}, 2 \mathrm{H}), 7.76-7.63(\mathrm{~m}, 4 \mathrm{H}), 7.46-7.12(\mathrm{~m}, 9 \mathrm{H}), 6.63(\mathrm{~s}, 1 \mathrm{H}), 4.33-4.05(\mathrm{~m}, 7 \mathrm{H}), 3.82(\mathrm{~s}, 3 \mathrm{H}), 2.77(\mathrm{t}, J=$ $7.6 \mathrm{~Hz}, 2 \mathrm{H}), 2.11-1.98(\mathrm{~m}, 3 \mathrm{H}) .{ }^{13} \mathrm{C}$ NMR $\left(101 \mathrm{MHz}, \mathrm{DMSO}-d_{6}\right) \delta 167.02,160.64,160.54,156.66,144.34$, $141.90,141.16,131.82,128.85,128.79,128.07,127.54,126.29,125.69,120.58,119.31,111.72,97.20,67.65$, 66.06, 56.56, 47.14, 31.92, 30.83. HRMS (ESI ${ }^{+}$: Calcd for $\mathrm{C}_{33} \mathrm{H}_{31} \mathrm{NO}_{6} \mathrm{Na}^{+}[\mathrm{M}+\mathrm{Na}]^{+}$560.2044, found 560.2042.

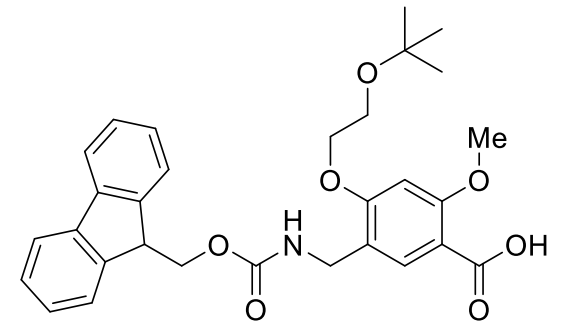

\section{5-(Fmoc-aminomethyl)-4-(2-(tert-butoxy)ethoxy)-2-methoxybenzoic acid} (4g, Fmoc-Mmb7(OtBu)-OH)

According to general procedure (c): 5-formyl-4-(2-(tert-butoxy)ethoxy)-2-methoxybenzoic acid 3g (1.5 g, 5.1 $\mathrm{mmol}$ ) gave a crude product that was purified by flash chromatography (ethyl acetate/hexanes) to give the title compound $4 \mathrm{~g}$ as a white powder $(2.2 \mathrm{~g}, 83 \%)$. ${ }^{1} \mathrm{H}$ NMR $(400 \mathrm{MHz}$, Chloroform- $d) \delta 8.10(\mathrm{~s}, 1 \mathrm{H}), 7.75(\mathrm{~d}, J=$ $7.5 \mathrm{~Hz}, 2 \mathrm{H}), 7.59(\mathrm{~d}, J=7.5 \mathrm{~Hz}, 2 \mathrm{H}), 7.38(\mathrm{t}, J=7.4 \mathrm{~Hz}, 2 \mathrm{H}), 7.29(\mathrm{t}, J=7.4 \mathrm{~Hz}, 4 \mathrm{H}), 6.57(\mathrm{~s}, 1 \mathrm{H}), 5.48(\mathrm{~d}, J=$ $6.3 \mathrm{~Hz}, 1 \mathrm{H}), 4.37(\mathrm{t}, J=6.7 \mathrm{~Hz}, 4 \mathrm{H}), 4.20(\mathrm{t}, J=4.9 \mathrm{~Hz}, 3 \mathrm{H}), 4.06(\mathrm{~s}, 3 \mathrm{H}), 3.81-3.73(\mathrm{~m}, 2 \mathrm{H}), 1.24(\mathrm{~d}, J=2.3$ $\mathrm{Hz}, 9 \mathrm{H}) .{ }^{13} \mathrm{C}$ NMR (101 MHz, Chloroform- $d$ ) $\delta 164.92,162.32,159.26,156.32,144.02,141.28,134.21$, 127.61, 127.01, 125.14, 121.60, 119.91, 109.74, 96.12, 73.72, 68.87, 66.78, 60.41, 60.23, 56.87, 47.23, 40.25, 28.76, 27.47, 21.06, 14.21. HRMS (ESI ${ }^{+}$): Calcd for $\mathrm{C}_{30} \mathrm{H}_{33} \mathrm{NO}_{7} \mathrm{Na}^{+}[\mathrm{M}+\mathrm{Na}]^{+}$542.2149, found 542.2148.

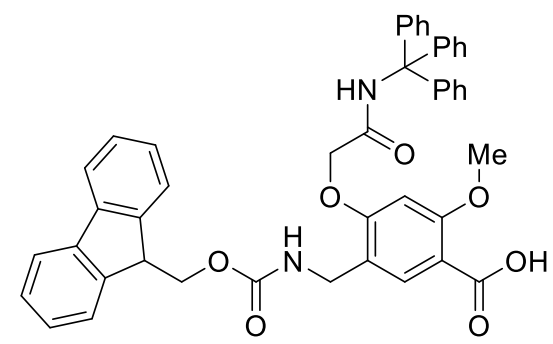

\section{5-(Fmoc-aminomethyl)-4-(2-oxo-2-(tritylamino)ethoxy)-2-methoxybenzoic acid}

\section{(4h, Fmoc-Mmb8(NHTr)-OH)}

According to general procedure (c): 5-formyl-4-(2-oxo-2-(tritylamino)ethoxy)-2-methoxybenzoic acid 3h (0.5 $\mathrm{g}, 5.1 \mathrm{mmol}$ ) gave a crude product that was purified by flash chromatography (ethyl acetate/hexanes) to give the title compound $\mathbf{4 h}$ as a white powder $(0.58 \mathrm{~g}, 66 \%) .{ }^{1} \mathrm{H}$ NMR $\left(400 \mathrm{MHz}, \mathrm{DMSO}-d_{6}\right) \delta 12.23(\mathrm{~s}, 1 \mathrm{H}), 8.70(\mathrm{~s}$, 1H), $7.89(\mathrm{~d}, J=7.5 \mathrm{~Hz}, 2 \mathrm{H}), 7.75(\mathrm{t}, J=6.0 \mathrm{~Hz}, 1 \mathrm{H}), 7.67(\mathrm{~d}, J=7.4 \mathrm{~Hz}, 2 \mathrm{H}), 7.65(\mathrm{~s}, 1 \mathrm{H}), 7.41(\mathrm{t}, J=7.3 \mathrm{~Hz}$, 
2H), $7.37-7.12(\mathrm{~m}, 19 \mathrm{H}), 6.61(\mathrm{~s}, 1 \mathrm{H}), 4.85(\mathrm{~s}, 2 \mathrm{H}), 4.26-4.12(\mathrm{~m}, 5 \mathrm{H}), 3.79(\mathrm{~s}, 3 \mathrm{H}) .{ }^{13} \mathrm{C} \mathrm{NMR}(101 \mathrm{MHz}$, DMSO- $\left.d_{6}\right) \delta 167.16,166.96,160.22,159.57,156.68,144.98,144.32,141.16,128.86,128.09,127.54,127.09$, 125.70, 120.57, 119.45, 112.61, 97.60, 69.79, 67.87, 66.06, 56.51, 47.10, 38.61, 1.63. HRMS (ESI ${ }^{+}$): Calcd for $\mathrm{C}_{45} \mathrm{H}_{38} \mathrm{~N}_{2} \mathrm{O}_{7} \mathrm{Na}^{+}[\mathrm{M}+\mathrm{Na}]^{+}$741.2571, found 741.2571.
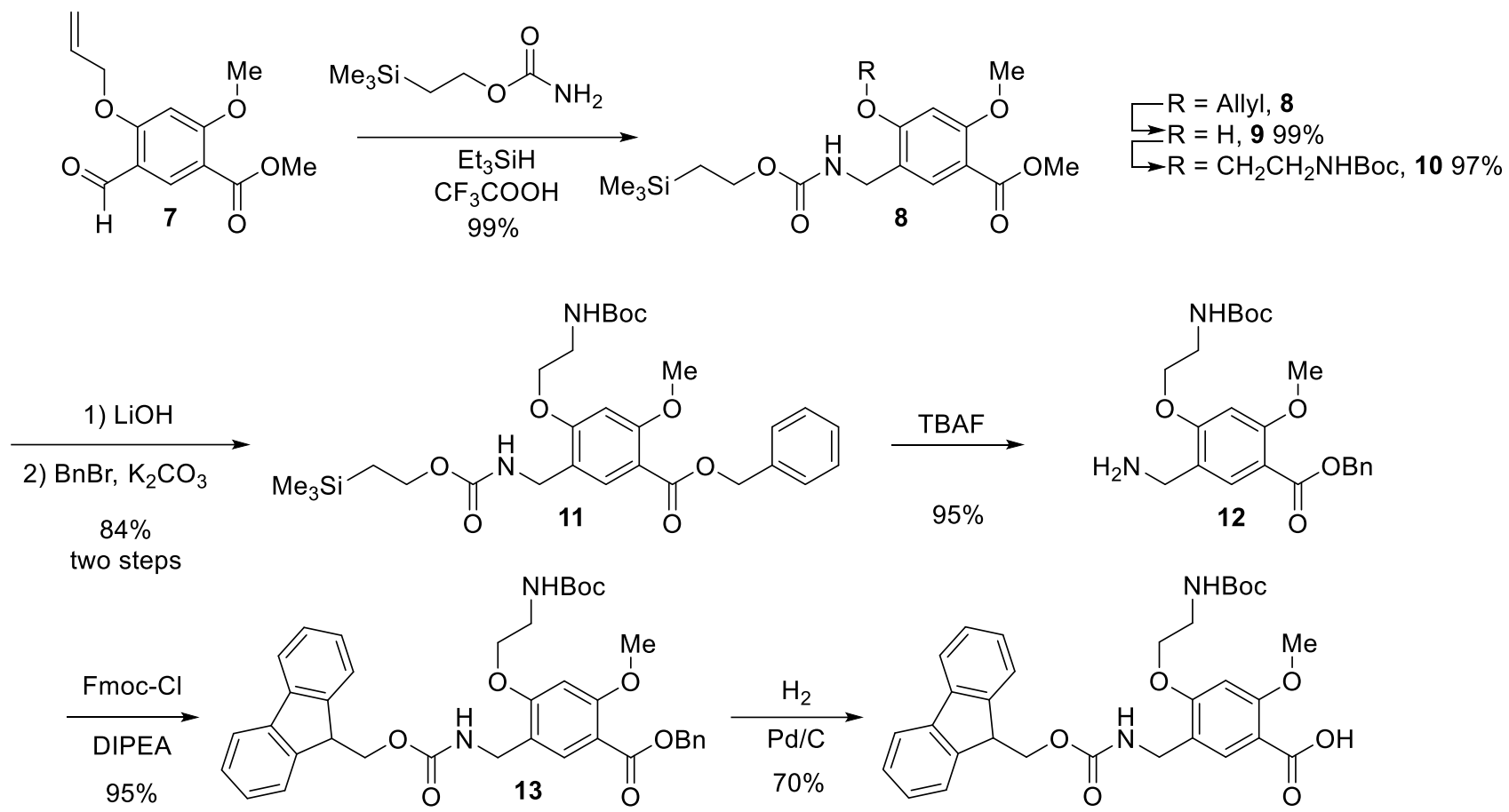

Figure S2: Synthesis of 4i, Fmoc-Mmb9(NHBoc)-OH

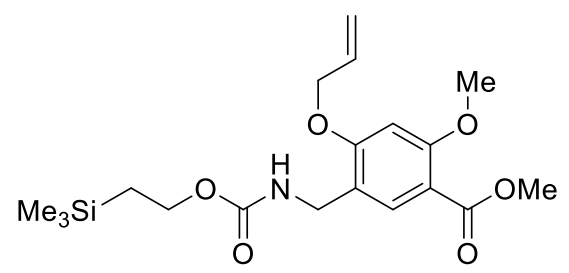

methyl 5-(Teoc-aminomethyl)-4-allyloxy-2-methoxybenzoate (8)

According to general procedure (c): methyl 5-formyl-4-allyloxy-2-methoxybenzoate 7 (2.8 g, $11 \mathrm{mmol})$ and 2(trimethylsilyl)ethyl carbamate (Teoc- $\mathrm{NH}_{2}, 2.2 \mathrm{~g}, 13 \mathrm{mmol}$ ) gave a crude product that was purified by flash chromatography (ethyl acetate/hexanes) to give the title compound $\mathbf{8}$ as a white powder (4.4 g, 99\%). ${ }^{1} \mathrm{H} \mathrm{NMR}$ $(400 \mathrm{MHz}$, Chloroform- $d$ ) $\delta 7.79$ (s, 1H), 6.42 (s, 1H), 6.03 (ddt, $J=17.3,10.5,5.2 \mathrm{~Hz}, 1 \mathrm{H}), 5.41$ (dq, $J=17.2$, $1.6 \mathrm{~Hz}, 1 \mathrm{H}), 5.31(\mathrm{dq}, J=10.5,1.4 \mathrm{~Hz}, 1 \mathrm{H}), 5.00(\mathrm{~s}, 1 \mathrm{H}), 4.60(\mathrm{dt}, J=5.3,1.6 \mathrm{~Hz}, 2 \mathrm{H}), 4.29(\mathrm{~d}, J=5.8 \mathrm{~Hz}$, $2 \mathrm{H}), 4.18-4.11(\mathrm{~m}, 2 \mathrm{H}), 3.87(\mathrm{~s}, 3 \mathrm{H}), 3.82(\mathrm{~s}, 3 \mathrm{H}), 1.02-0.88(\mathrm{~m}, 2 \mathrm{H}) .{ }^{13} \mathrm{C}$ NMR $(101 \mathrm{MHz}$, Chloroform- $d) \delta$ 165.80, 161.09, 160.76, 156.67, 133.18, 132.33, 118.99, 118.21, 111.49, 96.49, 69.08, 63.03, 56.26, 51.70, 40.09, 17.76, -1.47. HRMS (ESI ${ }^{+}$: Calcd for $\mathrm{C}_{19} \mathrm{H}_{29} \mathrm{NO}_{6} \mathrm{SiNa}^{+}[\mathrm{M}+\mathrm{Na}]^{+} 418.1656$, found 418.1652.<smiles>COC(=O)c1cc(CNC(=O)OCC[SiH2]C)c(O)cc1OC</smiles>

methyl 5-(Teoc-aminomethyl)-4-hydroxy-2-methoxybenzoate (9) 
To a solution of methyl 5-(Teoc-aminomethyl)-4-allyloxy-2-methoxybenzoate $\mathbf{8}$ (4.4 g, $11 \mathrm{mmol})$ and potassium carbonate $(4.6 \mathrm{~g}, 033 \mathrm{mmol}))$ in methanol $(50 \mathrm{~mL})$ was added tetrakis(triphenylphosphine)palladium(0) $(0.13 \mathrm{~g}, 0.11 \mathrm{mmol})$ and brought to reflux while stirring for $3 \mathrm{~h}$. The reaction mixture was then concentrated in vacuo, poured into ethyl acetate $(75 \mathrm{~mL})$ and the organic layer was washed with $1 \mathrm{M}$ sodium bisulfate $(2 \times 75 \mathrm{~mL})$, and brine $(1 \times 75 \mathrm{~mL})$, and then filtered over celite and magnesium sulfate and concentrated in vacuo to give the title compound $\mathbf{9}$ as an off-white solid (3.9 g, 99\%). The product was carried forward to the next step without further purification. ${ }^{1} \mathrm{H}$ NMR (400 MHz, Chloroformd) $\delta 9.71(\mathrm{~s}, 1 \mathrm{H}), 7.69(\mathrm{~s}, 1 \mathrm{H}), 7.52-7.35(\mathrm{~m}, 1 \mathrm{H}), 6.55(\mathrm{~s}, 1 \mathrm{H}), 5.49-5.33(\mathrm{~m}, 1 \mathrm{H}), 4.26-4.16(\mathrm{~m}, 4 \mathrm{H}), 3.91$ $-3.73(\mathrm{~m}, 6 \mathrm{H}), 1.04-0.89(\mathrm{~m}, 2 \mathrm{H}), 0.07-0.01(\mathrm{~m}, 9 \mathrm{H}) .{ }^{13} \mathrm{C}$ NMR $(101 \mathrm{MHz}$, Chloroform-d) $\delta 166.11$, $161.68,161.25,159.57,135.32,116.85,110.82,101.48,64.76,56.06,51.74,40.80,20.49,17.71,-1.51$. HRMS $\left(\mathrm{ESI}^{+}\right)$: Calcd for $\mathrm{C}_{16} \mathrm{H}_{25} \mathrm{NO}_{6} \mathrm{SiNa}^{+}[\mathrm{M}+\mathrm{Na}]^{+}$378.1343, found 378.1340.

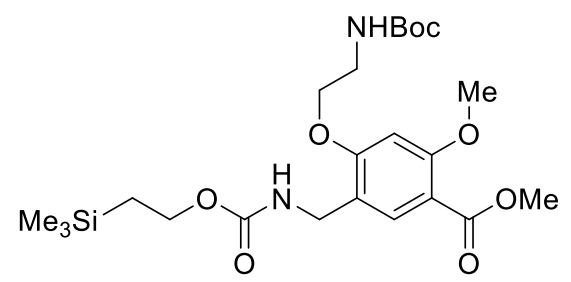

methyl 5-(Teoc-aminomethyl)-4-(2-(N-Boc-amino)ethoxy)-2-methoxybenzoate (10)

To a solution of methyl 5-(Teoc-aminomethyl)-4-hydroxy-2-methoxybenzoate 9 (3.9 g, $11 \mathrm{mmol})$ and potassium carbonate $(3.0 \mathrm{~g}, 22 \mathrm{mmol})$ in dimethylformamide $(30 \mathrm{~mL})$ was added tert-butyl (2bromoethyl)carbamate $(3.7 \mathrm{~g}, 17 \mathrm{mmol})$. The reaction mixture was stirred at $50{ }^{\circ} \mathrm{C}$ for $4 \mathrm{~h}$ poured into ethyl acetate $(100 \mathrm{~mL})$ and the organic layer was washed with $5 \%$ sodium bicarbonate $(3 \times 75 \mathrm{~mL})$, and brine $(1 \times 75$ $\mathrm{mL}$ ), and then filtered over celite and magnesium sulfate and concentrated in vacuo. The residue was purified by flash chromatography (ethyl acetate/hexanes) to give the title compound $\mathbf{1 0}$ as an off-white solid (5.3 g, 97\%). ${ }^{1} \mathrm{H}$ NMR (400 MHz, Chloroform-d) $\delta 7.78(\mathrm{~s}, 1 \mathrm{H}), 6.43(\mathrm{~s}, 1 \mathrm{H}), 5.47(\mathrm{~s}, 1 \mathrm{H}), 5.04(\mathrm{~d}, J=6.5 \mathrm{~Hz}, 1 \mathrm{H})$, $4.31(\mathrm{~d}, J=5.8 \mathrm{~Hz}, 2 \mathrm{H}), 4.22-4.13(\mathrm{~m}, 4 \mathrm{H}), 4.07(\mathrm{t}, J=4.9 \mathrm{~Hz}, 3 \mathrm{H}), 3.91(\mathrm{~s}, 3 \mathrm{H}), 3.85(\mathrm{~s}, 3 \mathrm{H}), 3.57(\mathrm{q}, J=5.4$ $\mathrm{Hz}, 2 \mathrm{H}), 1.46$ (s, 9H), $0.97(\mathrm{t}, J=8.4 \mathrm{~Hz}, 2 \mathrm{H}), 0.03(\mathrm{~s}, 9 \mathrm{H}) .{ }^{13} \mathrm{C}$ NMR (101 MHz, Chloroform- $d$ ) $\delta 165.83$, 161.33, 161.12, 156.73, 156.19, 133.51, 118.83, 111.35, 96.01, 79.62, 67.90, 63.08, 56.31, 51.74, 40.08, 40.01, 28.43, 17.75, -1.44. HRMS (ESI ${ }^{+}$): Calcd for $\mathrm{C}_{23} \mathrm{H}_{38} \mathrm{~N}_{2} \mathrm{O}_{8} \mathrm{SiNa}^{+}[\mathrm{M}+\mathrm{Na}]^{+}$521.2290, found 521.2289.

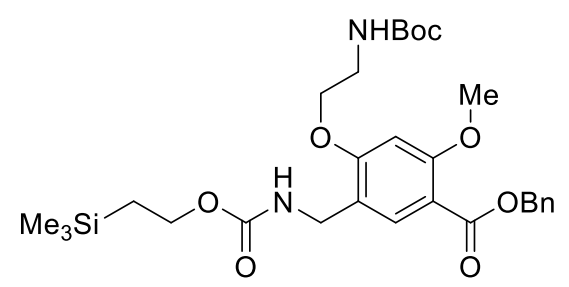

benzyl 5-(Teoc-aminomethyl)-4-(2-(N-Boc-amino)ethoxy)-2-methoxybenzoate (11)

According to general procedure (b): saponification of methyl 5-(Teoc-aminomethyl)-4-(2-(N-Bocamino)ethoxy)-2-methoxybenzoate 10 (5.3 g, $11 \mathrm{mmol})$ gave the desired acid as a colorless foam $(4.4 \mathrm{~g}, 86 \%)$. The product was used in the next step without further purification. To a solution of 5-(Teoc-aminomethyl)-4-(2( $N$-Boc-amino)ethoxy)-2-methoxybenzoic acid $(4.3 \mathrm{~g}, 8.9 \mathrm{mmol})$ and potassium carbonate $(2.5 \mathrm{~g}, 18 \mathrm{mmol}) \mathrm{in}$ dimethyl formamide $(30 \mathrm{~mL})$ was added benzyl bromide $(1.6 \mathrm{~mL}, 13 \mathrm{mmol})$ and stirred at room temperature for $4 \mathrm{~h}$ and then poured into ethyl acetate $(100 \mathrm{~mL})$ and the organic layer was washed with $5 \%$ sodium bicarbonate $(3 \times 75 \mathrm{~mL})$, and brine $(1 \times 75 \mathrm{~mL})$, and then filtered over celite and magnesium sulfate and concentrated in vасио. The residue was purified by flash chromatography (ethyl acetate/hexanes) to give the title compound 11 as an off-white solid (5.0 g, 98\%; 85\% over two steps. ${ }^{1} \mathrm{H}$ NMR (400 MHz, Chloroform- $d$ ) $\delta 7.79$ (s, $\left.1 \mathrm{H}\right), 7.47$ 
$-7.28(\mathrm{~m}, 5 \mathrm{H}), 6.43(\mathrm{~s}, 1 \mathrm{H}), 5.51(\mathrm{~d}, J=6.6 \mathrm{~Hz}, 1 \mathrm{H}), 5.31(\mathrm{~s}, 2 \mathrm{H}), 4.99(\mathrm{~s}, 1 \mathrm{H}), 4.30(\mathrm{~d}, J=5.6 \mathrm{~Hz}, 2 \mathrm{H}), 4.20-$ $4.14(\mathrm{~m}, 2 \mathrm{H}), 4.07(\mathrm{t}, J=5.0 \mathrm{~Hz}, 2 \mathrm{H}), 3.91(\mathrm{~s}, 3 \mathrm{H}), 3.56(\mathrm{q}, J=5.2 \mathrm{~Hz}, 2 \mathrm{H}), 1.45(\mathrm{~s}, 9 \mathrm{H}), 1.00-0.92(\mathrm{~m}, 2 \mathrm{H})$, 0.02 (s, 9H). ${ }^{13} \mathrm{C}$ NMR (101 MHz, Chloroform- $d$ ) $\delta 165.08,161.62,161.28,156.69,156.17,136.46,133.54$, 128.50, 128.10, 127.99, 118.78, 111.25, 96.04, 79.59, 67.92, 66.21, 63.08, 56.29, 53.43, 40.12, 40.03, 28.43, 17.74, -1.43. HRMS (ESI ${ }^{+}$: Calcd for $\mathrm{C}_{29} \mathrm{H}_{42} \mathrm{~N}_{2} \mathrm{O}_{8} \mathrm{SiNa}^{+}[\mathrm{M}+\mathrm{Na}]^{+}$597.2603, found 597.2590.

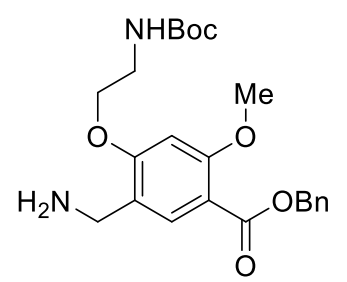

\section{benzyl 5-aminomethyl-4-(2-(N-Boc-amino)ethoxy)-2-methoxybenzoate (12)}

To a solution of benzyl 5-(Teoc-aminomethyl)-4-(2-(N-Boc-amino)ethoxy)-2-methoxybenzoate 11 (4.8 g, 8.4 $\mathrm{mmol})$ in tetrahydrofuran $(10 \mathrm{~mL})$ was added $1 \mathrm{M}$ tetrabutylammonium fluoride $(42 \mathrm{~mL}, 42 \mathrm{mmol})$ in tetrahydrofuran and stirred at room temperature until complete as indicated by consumption of starting material ( $4 \mathrm{~h}$, monitored by TLC). The reaction mixture was concentrated in vacuo and the residue was taken up in ethyl acetate $(100 \mathrm{~mL})$ and the organic layer was washed with $5 \%$ sodium bicarbonate $(3 \times 75 \mathrm{~mL})$, and brine $(1 \times 75$ $\mathrm{mL}$ ), and then filtered over celite and magnesium sulfate and concentrated in vacuo. The residue was purified by precipitation from cold dichloromethane to give the title compound $\mathbf{1 2}$ as a white powder $(3.4 \mathrm{~g}, 95 \%) .{ }^{1} \mathrm{H}$ NMR (400 MHz, DMSO- $\left.d_{6}\right) \delta 8.10-7.88(\mathrm{~m}, 2 \mathrm{H}), 7.83(\mathrm{~s}, 1 \mathrm{H}), 7.51-7.29(\mathrm{~m}, 5 \mathrm{H}), 7.24(\mathrm{t}, J=6.0 \mathrm{~Hz}, 1 \mathrm{H})$, $6.78(\mathrm{~s}, 1 \mathrm{H}), 5.28(\mathrm{~s}, 2 \mathrm{H}), 4.14(\mathrm{t}, J=5.2 \mathrm{~Hz}, 2 \mathrm{H}), 3.94(\mathrm{~s}, 2 \mathrm{H}), 3.90(\mathrm{~s}, 3 \mathrm{H}), 3.41(\mathrm{~d}, J=5.5 \mathrm{~Hz}, 2 \mathrm{H}), 1.39(\mathrm{~s}$, 9H). ${ }^{13} \mathrm{C}$ NMR $\left(101 \mathrm{MHz}, \mathrm{DMSO}-d_{6}\right) \delta 164.97,162.04,161.68,156.35,136.99,134.44,128.92,128.35$, 128.16, 114.39, 111.19, 97.49, 78.47, 68.75, 66.01, 56.81, 37.65, 28.72. HRMS (ESI ${ }^{+}$): Calcd for $\mathrm{C}_{23} \mathrm{H}_{31} \mathrm{~N}_{2} \mathrm{O}_{6}{ }^{+}$ $[\mathrm{M}+\mathrm{H}]^{+}$431.2177, found 431.2177.

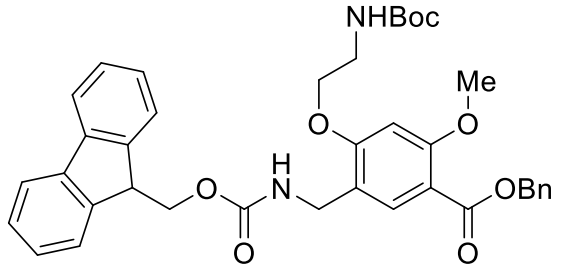

benzyl 5-(Fmoc-aminomethyl)-4-(2-(N-Boc-amino)ethoxy)-2-methoxybenzoate (13)

To a solution of benzyl 5-aminomethyl-4-(2-(N-Boc-amino)ethoxy)-2-methoxybenzoate 12 (3.1 g, $7.2 \mathrm{mmol})$ and diisopropylethylamine $(1.9 \mathrm{~mL}, 11 \mathrm{mmol})$ in dichloromethane $(30 \mathrm{~mL})$ was added 9-fluorenylmethyl chloroformate (Fmoc-Cl, $1.9 \mathrm{~g}, 7.2 \mathrm{mmol}$ ) and reaction mixture was stirred $30 \mathrm{~m}$ at room temperature. The reaction mixture was concentrated in vacuo and the residue was dissolved in ethyl acetate $(100 \mathrm{~mL})$ and the organic layer was washed with $1 \mathrm{M}$ sodium bisulfate $(3 \times 75 \mathrm{~mL})$ and brine $(1 \times 75 \mathrm{~mL})$, and then filtered over celite and magnesium sulfate and concentrated in vacuo. The residue was purified by flash chromatography (ethyl acetate/hexanes) to give the title compound 13 as an off-white solid (4.5 g, 95\%). ${ }^{1} \mathrm{H} \mathrm{NMR}(400 \mathrm{MHz}$, Chloroform- $d$ ) $\delta 7.81(\mathrm{~s}, 1 \mathrm{H}), 7.75(\mathrm{~d}, J=7.5 \mathrm{~Hz}, 2 \mathrm{H}), 7.58(\mathrm{~d}, J=7.5 \mathrm{~Hz}, 2 \mathrm{H}), 7.44(\mathrm{~d}, J=7.5 \mathrm{~Hz}, 2 \mathrm{H}), 7.42-$ $7.25(\mathrm{~m}, 9 \mathrm{H}), 6.43(\mathrm{~s}, 1 \mathrm{H}), 5.57(\mathrm{~s}, 1 \mathrm{H}), 5.31(\mathrm{~s}, 2 \mathrm{H}), 5.27(\mathrm{~s}, 1 \mathrm{H}), 4.39(\mathrm{~d}, J=7.2 \mathrm{~Hz}, 2 \mathrm{H}), 4.34(\mathrm{~d}, J=5.8 \mathrm{~Hz}$, $2 \mathrm{H}), 4.20(\mathrm{t}, J=7.2 \mathrm{~Hz}, 1 \mathrm{H}), 4.05(\mathrm{t}, J=4.8 \mathrm{~Hz}, 2 \mathrm{H}), 3.91(\mathrm{~s}, 3 \mathrm{H}), 3.53(\mathrm{q}, J=5.3 \mathrm{~Hz}, 2 \mathrm{H}), 1.38(\mathrm{~s}, 9 \mathrm{H}) .{ }^{13} \mathrm{C}$ NMR (101 MHz, Chloroform- $d$ ) $\delta$ 165.05, 161.74, 161.36, 156.35, 156.30, 143.96, 141.30, 136.44, 133.70, $128.50,128.13,128.01,127.67,127.01,125.12,119.96,118.60,111.24,96.05,79.57,67.99,66.82,66.23$, 56.31, 47.23, 40.25, 39.97, 28.38. HRMS (ESI ${ }^{+}$): Calcd for $\mathrm{C}_{38} \mathrm{H}_{40} \mathrm{~N}_{2} \mathrm{O}_{8} \mathrm{Na}^{+}[\mathrm{M}+\mathrm{Na}]^{+} 675.2677$, found 675.2668 . 


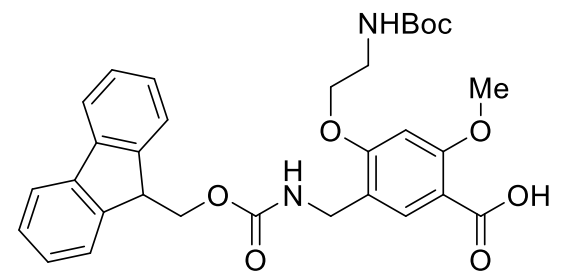

\section{5-(Fmoc-aminomethyl)-4-(2-(N-Boc-amino)ethoxy)-2-methoxybenzoic acid}

\section{(4i, Fmoc-Mmb9(NHBoc)-OH)}

A solution of benzyl 5-(Fmoc-aminomethyl)-4-(2-( $N$-Boc-amino)ethoxy)-2-methoxybenzoate 13 (4.0 g, 6.1 $\mathrm{mmol})$ and $10 \%$ palladium on activated carbon $(0.65 \mathrm{~g}, 0.61 \mathrm{mmol})$ in ethyl acetate $(30 \mathrm{~mL})$ was placed under a hydrogen atmosphere ( $\sim$ ATM balloon) and stirred for $16 \mathrm{~h}$. The reaction mixture was filtered through celite, concentrated in vacuo, and the residue was purified by flash chromatography (ethyl acetate/hexanes) to give the title compound $4 \mathbf{i}$ as a white powder $(2.4 \mathrm{~g}, 70 \%) .{ }^{1} \mathrm{H}$ NMR $\left(400 \mathrm{MHz}, \mathrm{DMSO}-d_{6}\right) \delta 12.13(\mathrm{~s}, 1 \mathrm{H}), 7.89(\mathrm{~d}, J=$ $7.5 \mathrm{~Hz}, 2 \mathrm{H}), 7.73-7.66(\mathrm{~m}, 3 \mathrm{H}), 7.64(\mathrm{~s}, 1 \mathrm{H}), 7.41(\mathrm{t}, J=7.4 \mathrm{~Hz}, 2 \mathrm{H}), 7.32(\mathrm{t}, J=7.4 \mathrm{~Hz}, 2 \mathrm{H}), 7.07(\mathrm{t}, J=6.0$ $\mathrm{Hz}, 1 \mathrm{H}), 6.67(\mathrm{~s}, 1 \mathrm{H}), 4.30(\mathrm{~d}, J=7.1 \mathrm{~Hz}, 2 \mathrm{H}), 4.23(\mathrm{t}, J=7.0 \mathrm{~Hz}, 1 \mathrm{H}), 4.13(\mathrm{~d}, J=6.0 \mathrm{~Hz}, 2 \mathrm{H}), 4.07(\mathrm{t}, J=5.4$ $\mathrm{Hz}, 2 \mathrm{H}), 3.85$ (s, 3H), 3.37 (q, $J=5.5 \mathrm{~Hz}, 2 \mathrm{H}), 1.37(\mathrm{~s}, 9 \mathrm{H}) .{ }^{13} \mathrm{C}$ NMR (101 MHz, DMSO- $\left.d 6\right) \delta 166.99,160.54$, 156.69, 156.26, 144.33, 141.18, 131.89, 128.08, 127.54, 125.66, 120.58, 119.45, 111.79, 97.30, 78.33, 68.09, 66.02, 56.59, 55.39, 47.16, 38.91, 28.66. HRMS (ESI ${ }^{+}$): Calcd for $\mathrm{C}_{31} \mathrm{H}_{34} \mathrm{~N}_{2} \mathrm{O}_{8} \mathrm{Na}^{+}[\mathrm{M}+\mathrm{Na}]^{+}$585.2207, found 585.2208 .
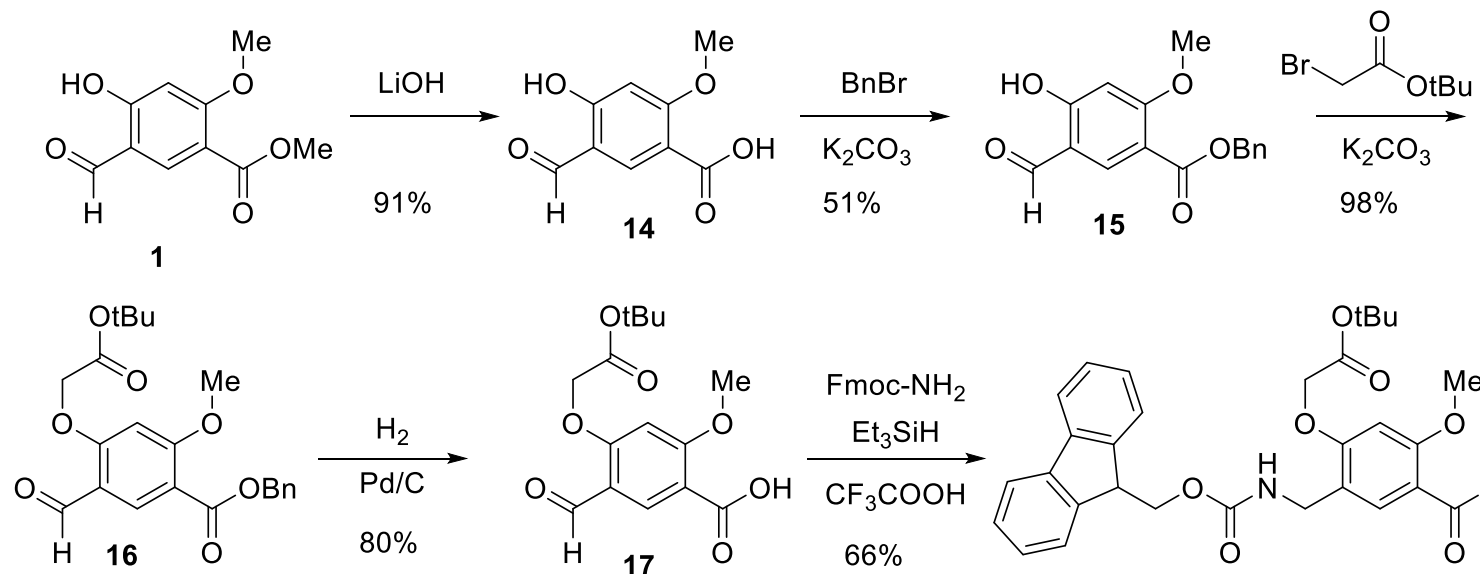

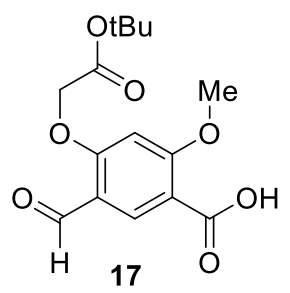

17

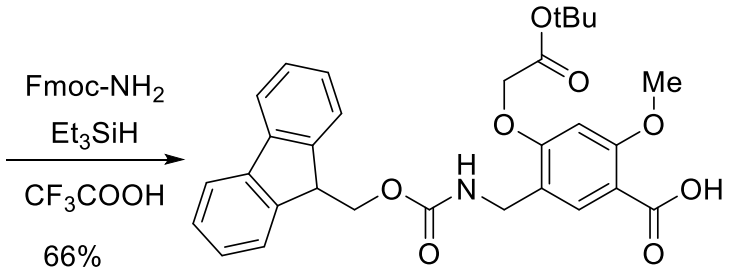

4j, Fmoc-Mmb10(OtBu)-OH

Figure S3: Synthesis of 4j, Fmoc-Mmb10(OtBu)-OH<smiles>COc1cc(O)c(C=O)cc1C(=O)O</smiles>

\section{5-formyl-4-hydroxy-2-methoxybenzoic acid (14)}

According to general procedure (b): saponification of methyl 5-formyl-4-hydroxy-2-methoxybenzoate 1 (2.0 g, $10 \mathrm{mmol}$ ) afforded the desired acid by precipitation upon acidification. The precipitate was filtered, washed with diethyl ether, and dried to give the title compound as a white crystalline powder $(1.7 \mathrm{~g}, 91 \%) .{ }^{1} \mathrm{H} \mathrm{NMR}$ $\left(400 \mathrm{MHz}, \mathrm{DMSO}-d_{6}\right) \delta 12.51(\mathrm{~s}, 1 \mathrm{H}), 11.48(\mathrm{~s}, 1 \mathrm{H}), 10.07(\mathrm{~s}, 1 \mathrm{H}), 8.14(\mathrm{~s}, 1 \mathrm{H}), 6.61(\mathrm{~s}, 1 \mathrm{H}), 3.87(\mathrm{~s}, 3 \mathrm{H}) .{ }^{13} \mathrm{C}$ NMR (101 MHz, DMSO-d $\left.d_{6}\right) \delta 190.66,166.09,165.78,165.62,135.29,115.86,113.32,100.34$, 56.67. HRMS $\left(\mathrm{ESI}^{+}\right)$: Calcd for $\mathrm{C}_{9} \mathrm{H}_{8} \mathrm{O}_{5} \mathrm{Na}^{+}[\mathrm{M}+\mathrm{Na}]^{+} 219.0264$, found 219.0263 . 


\section{benzyl 5-formyl-4-hydroxy-2-methoxybenzoate (15)}

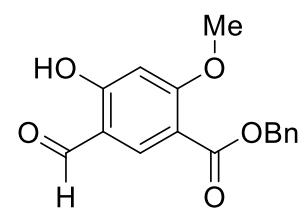

To a solution of 5-formyl-4-hydroxy-2-methoxybenzoic acid $\mathbf{1 4}(1.0 \mathrm{~g}, 5.1 \mathrm{mmol})$ and sodium bicarbonate $(0.45$ $\mathrm{g}, 5.4 \mathrm{mmol})$ in dimethyl formamide $(15 \mathrm{~mL})$ was added benzyl bromide $(0.64 \mathrm{~mL}, 5.4 \mathrm{mmol})$ and stirred at room temperature for $4 \mathrm{~h}$ and then poured into ethyl acetate $(100 \mathrm{~mL})$ and the organic layer was washed with 1 M sodium bisulfate $(3 \times 75 \mathrm{~mL})$, and brine $(1 \times 75 \mathrm{~mL})$, and then filtered over celite and magnesium sulfate and concentrated in vacuo. The residue was purified by flash chromatography (ethyl acetate/hexanes) to give the title compound 15 as white solid (0.75 g, 51\%). ${ }^{1} \mathrm{H}$ NMR (400 MHz, Chloroform- $d$ ) $\delta 11.69$ (s, 1H), 9.72 (s, $1 \mathrm{H}), 8.16(\mathrm{~s}, 1 \mathrm{H}), 7.49-7.30(\mathrm{~m}, 5 \mathrm{H}), 6.48(\mathrm{~s}, 1 \mathrm{H}), 5.33(\mathrm{~s}, 2 \mathrm{H}), 3.95(\mathrm{~s}, 3 \mathrm{H}) .{ }^{13} \mathrm{C}$ NMR $(101 \mathrm{MHz}$, Chloroform- $d$ ) $\delta$ 194.52, 166.93, 166.51, 163.99, 139.41, 136.03, 128.59, 128.24, 128.20, 114.28, 112.74, 100.02, 66.63, 56.56. HRMS (ESI ${ }^{+}$: Calcd for $\mathrm{C}_{16} \mathrm{H}_{14} \mathrm{O}_{5} \mathrm{Na}^{+}[\mathrm{M}+\mathrm{Na}]^{+}$309.0733, found 309.0737.<smiles>CCCCOC(=O)COc1cc(OC)c(C(=O)OCCCC)cc1C=O</smiles>

benzyl 4-(2-(tert-butoxy)-2-oxoethoxy)-5-formyl-2-methoxybenzoate (16)

According to general procedure (a): benzyl 5-formyl-4-hydroxy-2-methoxybenzoate 15 ( $0.70 \mathrm{~g}, 2.5 \mathrm{mmol})$, potassium carbonate $(0.68 \mathrm{~g}, 4.9 \mathrm{mmol})$, and tert-butyl bromoacetate $(0.54 \mathrm{~mL}, 3.7 \mathrm{mmol})$ gave the title compound 16 as a white solid $(0.96 \mathrm{~g}, 98 \%)$. ${ }^{1} \mathrm{H}$ NMR (400 MHz, Chloroform- $d$ ) $\delta 10.36(\mathrm{~s}, 1 \mathrm{H}), 8.46(\mathrm{~s}, 1 \mathrm{H})$, $7.47-7.29(\mathrm{~m}, 5 \mathrm{H}), 6.33(\mathrm{~s}, 1 \mathrm{H}), 5.32(\mathrm{~s}, 2 \mathrm{H}), 4.69(\mathrm{~s}, 2 \mathrm{H}), 3.95(\mathrm{~s}, 3 \mathrm{H}), 1.49(\mathrm{~s}, 9 \mathrm{H}) .{ }^{13} \mathrm{C} \mathrm{NMR}(101 \mathrm{MHz}$, Chloroform- $d$ ) $\delta 187.34,166.51,165.61,164.32,164.19,136.00,133.95,128.56,128.28,128.18,118.27$, 113.77, 95.82, 83.38, 66.61, 66.12, 56.32, 28.03. HRMS (ESI ${ }^{+}$): Calcd for $\mathrm{C}_{22} \mathrm{H}_{24} \mathrm{O}_{7} \mathrm{Na}^{+}[\mathrm{M}+\mathrm{Na}]^{+} 423.1414$, found 423.1417.<smiles>CCCC(=O)COc1cc(OC)c(C(=O)O)cc1C=O</smiles>

4-(2-(tert-butoxy)-2-oxoethoxy)-5-formyl-2-methoxybenzoic acid (17)

According to general procedure (b): saponification of benzyl 4-(2-(tert-butoxy)-2-oxoethoxy)-5-formyl-2methoxybenzoate $16(0.90 \mathrm{~g}, 2.3 \mathrm{mmol})$ gave the title compound 17 as a white solid $(0.56 \mathrm{~g}, 80 \%) .{ }^{1} \mathrm{H}$ NMR $(400 \mathrm{MHz}$, Chloroform- $d$ ) $\delta 11.72(\mathrm{~s}, 1 \mathrm{H}), 9.77(\mathrm{~s}, 1 \mathrm{H}), 8.28(\mathrm{~s}, 1 \mathrm{H}), 6.50(\mathrm{~s}, 1 \mathrm{H}), 4.71(\mathrm{~s}, 2 \mathrm{H}), 3.97(\mathrm{~s}, 3 \mathrm{H})$, $1.50(\mathrm{~s}, 10 \mathrm{H}) .{ }^{13} \mathrm{C}$ NMR $(101 \mathrm{MHz}$, Chloroform- $d$ ) $\delta 194.54,167.20,166.97,166.68,163.22,139.83,114.36$, 111.97, 100.05, 82.57, 61.36, 56.65, 28.08. HRMS (ESI ${ }^{+}$): Calcd for $\mathrm{C}_{15} \mathrm{H}_{18} \mathrm{O}_{7} \mathrm{Na}^{+}[\mathrm{M}+\mathrm{Na}]^{+} 333.0945$, found 333.0942 . 


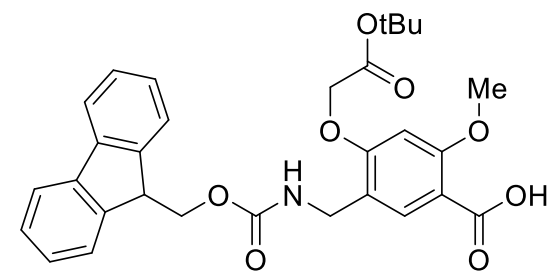

\section{5-(Fmoc-aminomethyl)-4-(2-(tert-butoxy)-2-oxoethoxy)-2-methoxybenzoic acid}

\section{$(4 j, \mathrm{Fmoc}-\mathrm{Mmb10}(\mathrm{OtBu})-\mathrm{OH})$}

According to general procedure (c): 4-(2-(tert-butoxy)-2-oxoethoxy)-5-formyl-2-methoxybenzoic acid 17 (0.55 $\mathrm{g}, 1.8 \mathrm{mmol}$ ) gave a crude product that was purified by flash chromatography (ethyl acetate/hexanes) to give the title compound 4j as a white powder $(0.62 \mathrm{~g}, 66 \%) .{ }^{1} \mathrm{H}$ NMR $(400 \mathrm{MHz}$, Chloroform- $d) \delta 9.53(\mathrm{~s}, 1 \mathrm{H}), 8.10(\mathrm{~s}$, $1 \mathrm{H}), 7.74(\mathrm{~d}, J=7.5 \mathrm{~Hz}, 2 \mathrm{H}), 7.61(\mathrm{~d}, J=7.5 \mathrm{~Hz}, 2 \mathrm{H}), 7.37(\mathrm{t}, J=7.5 \mathrm{~Hz}, 2 \mathrm{H}), 7.32-7.24(\mathrm{~m}, 2 \mathrm{H}), 6.38(\mathrm{~s}$, $1 \mathrm{H}), 5.91(\mathrm{t}, J=6.1 \mathrm{~Hz}, 1 \mathrm{H}), 4.66(\mathrm{~s}, 2 \mathrm{H}), 4.41(\mathrm{~d}, J=6.1 \mathrm{~Hz}, 2 \mathrm{H}), 4.36(\mathrm{~d}, J=7.2 \mathrm{~Hz}, 2 \mathrm{H}), 4.19(\mathrm{t}, J=7.2 \mathrm{~Hz}$, 1H), 4.00 (s, 3H), 1.49 (s, 9H). ${ }^{13} \mathrm{C}$ NMR (101 MHz, Chloroform- $d$ ) $\delta$ 167.10, 165.20, 161.01, 159.40, 156.40, $144.05,141.25,134.72,127.60,127.02,125.20,121.49,119.88,110.64,95.76,83.42,66.78,65.95,56.83$, 47.24, 40.32, 28.04. HRMS (ESI ${ }^{+}$: Calcd for $\mathrm{C}_{30} \mathrm{H}_{31} \mathrm{NO}_{8} \mathrm{Na}^{+}[\mathrm{M}+\mathrm{Na}]^{+}$556.1942, found 556.1943.

\subsection{Solid Phase Synthesis Procedures}

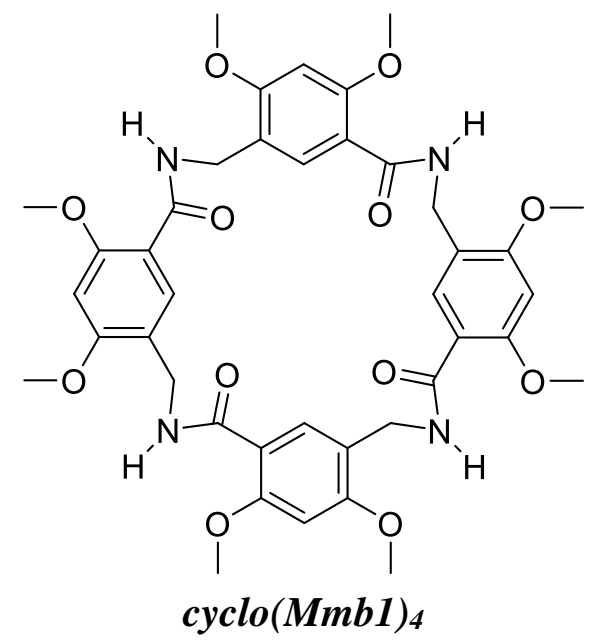

$1^{4}, 1^{6}, 5^{4}, 5^{6}, 9^{4}, 9^{6}, 13^{4}, 13^{6}$-octamethoxy-3,7,11,15-tetraaza-1,5,9,13(1,3)-tetrabenzenacyclohexadecaphane2,6,10,14-tetraone

According to general procedure (d): $\mathrm{H}-(\mathrm{Mmb} 1)_{4}-\mathrm{OH}$ was prepared from monomer Fmoc-Mmb1-OH, 4a using 2-chlorotrityl chloride resin on a $30 \mu \mathrm{mol}$ scale $(21 \mathrm{mg}, 89 \%) . \mathrm{H}-(\mathrm{Mmb} 1)_{4}-\mathrm{OH}(18 \mathrm{mg}, 23 \mu \mathrm{mol})$ was cyclized according to general procedure (e) and the residue was purified by precipitation from cold water and collected by centrifugation. The pellet was resuspended in cold methanol and the precipitate was collected by centrifugation and dried in vacuo to give the title compound as a white powder (12 $\mathrm{mg}$, 69\% cyclization, $61 \%$ overall from resin loading). ${ }^{1} \mathrm{H}$ NMR $\left(600 \mathrm{MHz}, \mathrm{DMSO}-d_{6}\right) \delta 8.49(\mathrm{t}, J=6.2 \mathrm{~Hz}, 4 \mathrm{H}), 7.65(\mathrm{~s}, 4 \mathrm{H}), 6.71(\mathrm{~s}$, $4 \mathrm{H}), 4.29$ (d, $J=6.0 \mathrm{~Hz}, 8 \mathrm{H}), 3.99$ (s, 12H), $3.94(\mathrm{~s}, 12 \mathrm{H}) .{ }^{13} \mathrm{C}$ NMR $\left(151 \mathrm{MHz}, \mathrm{DMSO}-d_{6}\right) \delta 163.75,160.00$, 157.89, 130.62, 119.59, 112.44, 95.78, 56.23, 55.76, 37.86. MS (MALDI-TOF ${ }^{+}$) Calcd for $\mathrm{C}_{40} \mathrm{H}_{43} \mathrm{~N}_{4} \mathrm{O}_{12} \mathrm{Na}^{\cdot+}$ $[\mathrm{M}+\mathrm{Na}]^{+} 794.3$, found 794.7 . 


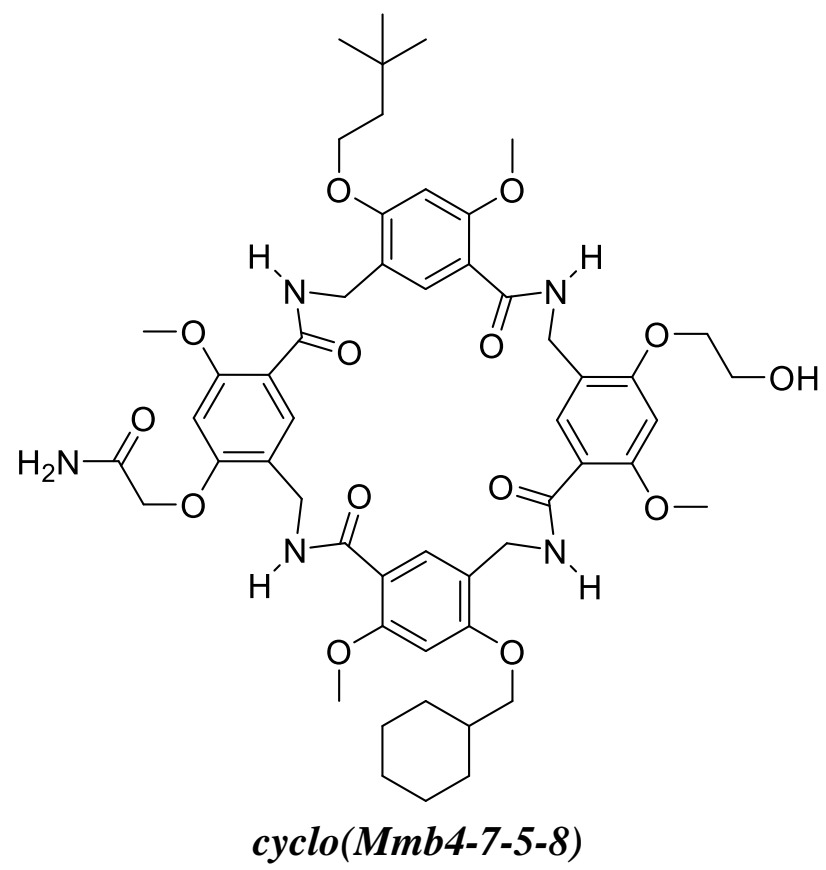

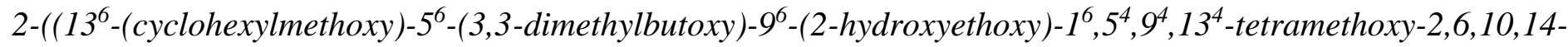
tetraoxo-3,7,11,15-tetraaza-1,5,9,13(1,3)-tetrabenzenacyclohexadecaphane-1 ${ }^{4}$-yl)oxy)acetamide

According to general procedure (d): $\mathrm{H}-\mathrm{Mmb} 4-7(\mathrm{OtBu})-5-8(\mathrm{Tr})-\mathrm{OH}$ was prepared on a $30 \mu \mathrm{mol}$ scale by loading Fmoc-Mmb8(OTr)-OH, $\mathbf{4 h}$ onto 2-chlorotrityl chloride resin and coupling $\mathbf{4 e}, \mathbf{4 g}$, and $\mathbf{4 d}$ in succession $(37 \mathbf{m g}$, 94\%). H-Mmb4-7( $\mathrm{OtBu})-5-8(\mathrm{Tr})-\mathrm{OH}(20 \mathrm{mg}, 15 \mu \mathrm{mol})$ was cyclized according to general procedure (e) and the residue was purified by precipitation from cold water and collected by centrifugation and dried in vacuo. The crude material was deprotected according to general procedure (f), concentrated in vacuo, and purified by flash chromatography (methanol/methylene chloride) to give the title compound as a white powder $(11 \mathrm{mg}, 69 \%$ cyclization, $65 \%$ overall from resin loading). ${ }^{1} \mathrm{H}$ NMR $\left(600 \mathrm{MHz}, \mathrm{DMSO}-d_{6}\right) \delta 8.55(\mathrm{t}, J=6.2 \mathrm{~Hz}, 1 \mathrm{H}), 8.45$ (q, $J=6.7 \mathrm{~Hz}, 2 \mathrm{H}), 8.35(\mathrm{t}, J=6.2 \mathrm{~Hz}, 1 \mathrm{H}), 7.78(\mathrm{~s}, 1 \mathrm{H}), 7.71(\mathrm{~s}, 1 \mathrm{H}), 7.65(\mathrm{~d}, J=6.0 \mathrm{~Hz}, 2 \mathrm{H}), 7.62(\mathrm{~s}, 1 \mathrm{H}), 7.57$ $(\mathrm{s}, 1 \mathrm{H}), 6.71(\mathrm{~s}, 1 \mathrm{H}), 6.70(\mathrm{~s}, 1 \mathrm{H}), 6.69(\mathrm{~s}, 1 \mathrm{H}), 6.65(\mathrm{~s}, 1 \mathrm{H}), 4.99(\mathrm{t}, J=5.7 \mathrm{~Hz}, 1 \mathrm{H}), 4.62(\mathrm{~s}, 2 \mathrm{H}), 4.41(\mathrm{~d}, J=$ $6.1 \mathrm{~Hz}, 2 \mathrm{H}), 4.33(\mathrm{t}, J=6.4 \mathrm{~Hz}, 6 \mathrm{H}), 4.19(\mathrm{t}, J=6.9 \mathrm{~Hz}, 2 \mathrm{H}), 4.15(\mathrm{t}, J=4.9 \mathrm{~Hz}, 2 \mathrm{H}), 4.00-3.92(\mathrm{~m}, 15 \mathrm{H})$, $3.80(\mathrm{q}, J=5.3 \mathrm{~Hz}, 2 \mathrm{H}), 1.89-1.66(\mathrm{~m}, 9 \mathrm{H}), 1.36-1.09(\mathrm{~m}, 8 \mathrm{H}), 1.02(\mathrm{~s}, 5 \mathrm{H}) .{ }^{13} \mathrm{C}$ NMR (151 MHz, DMSO$\left.d_{6}\right) \delta 170.03,164.55,164.41,164.38,164.25,160.23,159.90,159.83,158.93,158.40,158.36,158.28,132.66$, $132.03,131.09,130.73,120.55,120.39,119.95,113.60,113.15,112.88,112.78,97.54,97.12,96.69,96.63$, 73.51, 70.67, 67.74, 65.98, 60.13, 56.77, 56.71, 56.65, 40.53, 38.68, 38.51, 38.39, 31.17, 30.10, 29.77, 26.53, 26.41, 25.84. MS (MALDI-TOF ${ }^{+}$) Calcd for $\mathrm{C}_{53} \mathrm{H}_{66} \mathrm{~N}_{5} \mathrm{O}_{14} \mathrm{Na}^{a+}[\mathrm{M}+\mathrm{Na}]^{+}$1019.4, found 1019.2. 


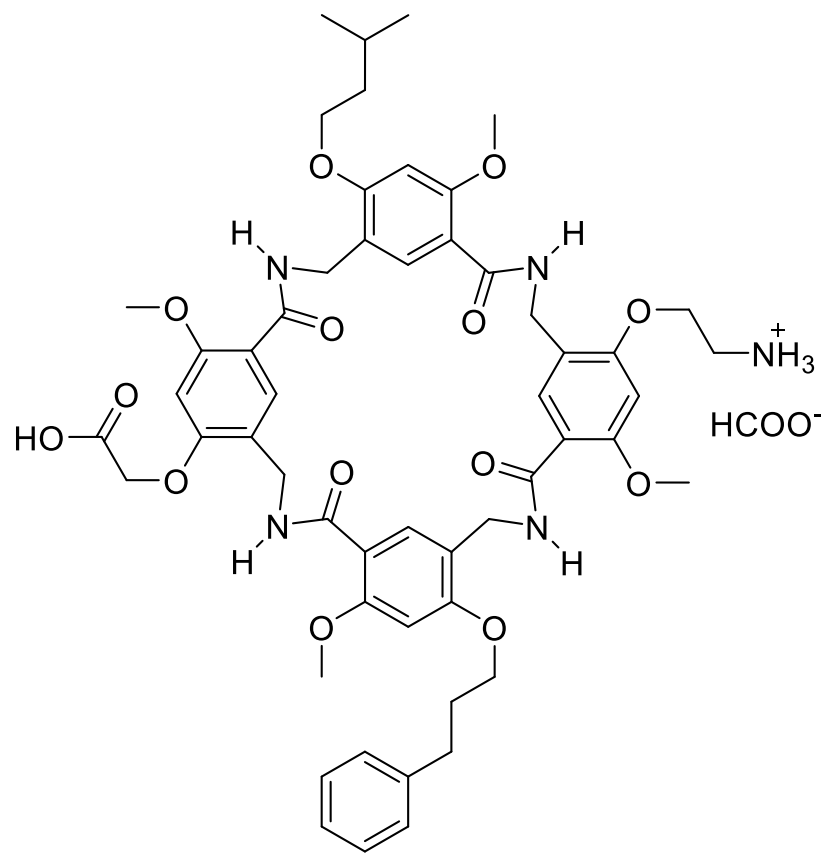

cyclo(Mmb3-9-6-10)・HCOOH

2-((96-(carboxymethoxy)-136-(isopentyloxy)- $1^{6}, 5^{4}, 9^{4}, 13^{4}$-tetramethoxy-2,6,10, 14-tetraoxo- $5^{6}-(3-$ phenylpropoxy)-3,7,11,15-tetraaza-1,5,9,13(1,3)-tetrabenzenacyclohexadecaphane-1 ${ }^{4}$-yl)oxy)ethan-1-aminium formate

According to general procedure (d): H-Mmb3-9(Boc)-6-10(OtBu)-OH was prepared on a $30 \mu$ mol scale by loading Fmoc-Mmb10(OtBu)-OH, $\mathbf{4 j}$ onto 2-chlorotrityl chloride resin and coupling $\mathbf{4 f}, \mathbf{4 i}$, and $\mathbf{4 c}$ in succession (31 mg, 89\%). H-Mmb3-9(Boc)-6-10(OtBu)-OH (20 mg, $17 \mu \mathrm{mol})$ was cyclized according to general procedure (e) and the residue was purified by precipitation from cold water and collected by centrifugation and dried in vасио. The crude material was deprotected according to general procedure (f), concentrated in vacuo, and purified by UHPLC (methanol/water/acetonitrile) to give the title compound (formate salt) as a colorless solid (12 mg, 69\% cyclization, $61 \%$ overall from resin loading). ${ }^{1} \mathrm{H}$ NMR (600 MHz, DMSO- $\left.d_{6}\right) \delta 8.71(\mathrm{t}, J=6.4$ $\mathrm{Hz}, 1 \mathrm{H}), 8.50(\mathrm{dt}, J=12.9,6.5 \mathrm{~Hz}, 2 \mathrm{H}), 8.32(\mathrm{t}, J=6.5 \mathrm{~Hz}, 1 \mathrm{H}), 8.05(\mathrm{t}, J=6.2 \mathrm{~Hz}, 3 \mathrm{H}), 7.84(\mathrm{~s}, 1 \mathrm{H}), 7.71(\mathrm{~s}$, 1H), $7.68(\mathrm{~s}, 1 \mathrm{H}), 7.67(\mathrm{~s}, 1 \mathrm{H}), 7.33-7.17(\mathrm{~m}, 5 \mathrm{H}), 6.74(\mathrm{~s}, 1 \mathrm{H}), 6.70(\mathrm{~s}, 1 \mathrm{H}), 6.69(\mathrm{~s}, 1 \mathrm{H}), 6.64(\mathrm{~s}, 1 \mathrm{H}), 4.88$ $(\mathrm{s}, 1 \mathrm{H}), 4.44-4.28(\mathrm{~m}, 8 \mathrm{H}), 4.14(\mathrm{dt}, J=13.1,6.8 \mathrm{~Hz}, 4 \mathrm{H}), 3.99-3.90(\mathrm{~m}, 13 \mathrm{H}), 3.33(\mathrm{q}, J=5.6 \mathrm{~Hz}, 2 \mathrm{H})$, $2.84-2.80(\mathrm{~m}, 2 \mathrm{H}), 2.13-2.10(\mathrm{~m}, 1 \mathrm{H}), 1.91-1.81(\mathrm{~m}, 1 \mathrm{H}), 1.71(\mathrm{q}, J=6.8 \mathrm{~Hz}, 2 \mathrm{H}), 0.98(\mathrm{~d}, J=6.9 \mathrm{~Hz}$, $6 \mathrm{H}) .{ }^{13} \mathrm{C}$ NMR (151 MHz, DMSO- $\left.d_{6}\right) \delta 169.70,163.87,163.66,158.57,158.40,157.84,157.66,141.25$, $128.28,125.78,120.26,119.94,119.54,119.36,113.25,113.02,112.29,96.93,96.75,96.04,96.02,66.95$, $66.38,65.05,65.01,56.33,56.31,56.13,56.09,39.94,37.72,37.37,31.38,30.32,24.60$, 22.40. MS (MALDI$\mathrm{TOF}^{+}$) Calcd for $\mathrm{C}_{54} \mathrm{H}_{62} \mathrm{~N}_{5} \mathrm{O}_{14} \mathrm{Na}^{\cdot+}[\mathrm{M}+\mathrm{Na}]^{+} 1027.4$, found 1027.2. 


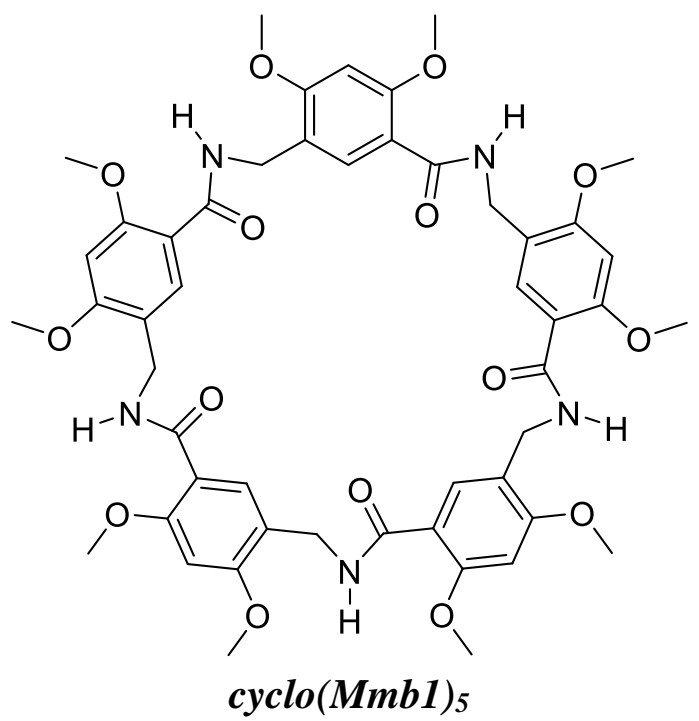

$1^{4}, 1^{6}, 5^{4}, 5^{6}, 9^{4}, 9^{6}, 13^{4}, 13^{6}, 17^{4}, 17^{6}$-decamethoxy-3,7,11,15,19-pentaaza-1,5,9,13,17(1,3)-

pentabenzenacycloicosaphane-2,6,10,14,18-pentaone

According to general procedure (d): $\mathrm{H}-(\mathrm{Mmb} 1)_{5}-\mathrm{OH}$ was prepared from monomer Fmoc-Mmb1-OH, 4a using 2-chlorotrityl chloride resin on a $30 \mu \mathrm{mol}$ scale $(27 \mathrm{mg}, 91 \%)$. H-(Mmb1) $)_{5}-\mathrm{OH}(22 \mathrm{mg}, 22 \mu \mathrm{mol})$ was cyclized according to general procedure (e) and the residue was purified by precipitation from cold water and collected by centrifugation. The pellet was resuspended in cold methanol and the precipitate was collected by centrifugation and dried in vacuo to give the title compound as a white powder (19 $\mathrm{mg}, 90 \%$ cyclization, $82 \%$ overall from resin loading). ${ }^{1} \mathrm{H}$ NMR $\left(600 \mathrm{MHz}\right.$, DMSO- $\left.d_{6}\right) \delta 8.45(\mathrm{t}, J=6.2 \mathrm{~Hz}, 5 \mathrm{H}), 7.69(\mathrm{~s}, 5 \mathrm{H}), 6.73$ (s, $5 \mathrm{H}), 4.36(\mathrm{~d}, J=6.1 \mathrm{~Hz}, 10 \mathrm{H}), 3.97(\mathrm{~s}, 15 \mathrm{H}), 3.95(\mathrm{~s}, 15 \mathrm{H}) .{ }^{13} \mathrm{C}$ NMR $\left(151 \mathrm{MHz}\right.$, DMSO- $\left.d_{6}\right) \delta 164.56,160.38$, 158.36, 130.56, 119.84, 113.71, 96.20, 56.82, 56.36, 37.91. MS (MALDI-TOF ${ }^{+}$Calcd for $\mathrm{C}_{50} \mathrm{H}_{54} \mathrm{~N}_{5} \mathrm{O}_{15} \mathrm{Na}^{*+}$ $[\mathrm{M}+\mathrm{Na}]^{+}$987.4, found 987.2.

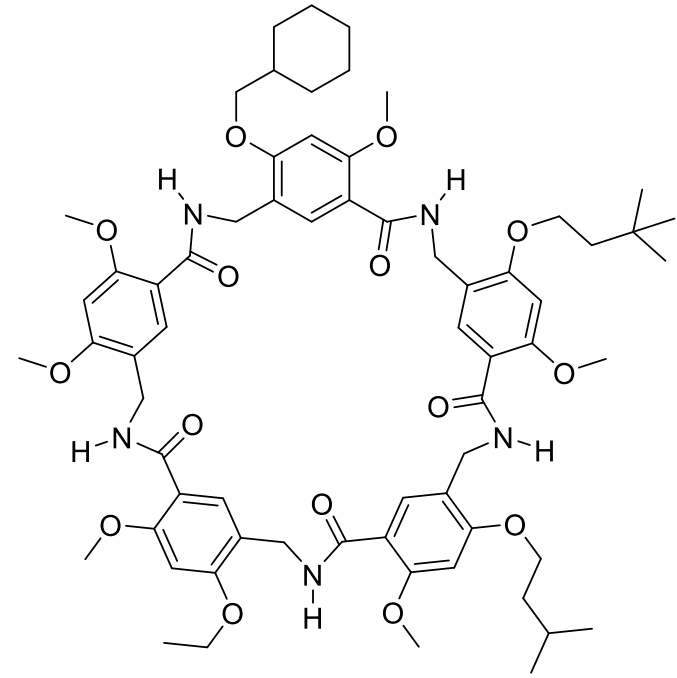

cyclo(Mmb5-4-3-2-1)

$1^{4}$-(cyclohexylmethoxy)-56-(3,3-dimethylbutoxy)-1 $13^{6}$-ethoxy-96-(isopentyloxy)- $1^{6}, 5^{4}, 9^{4}, 13^{4}, 17^{4}, 17^{6}$-hexamethoxy3,7,11,15,19-pentaaza-1,5,9,13,17(1,3)-pentabenzenacycloicosaphane-2,6,10,14,18-pentaone

According to general procedure (d): H-Mmb5-4-3-2-1-OH was prepared on a $30 \mu \mathrm{mol}$ scale by loading FmocMmb1-OH, 4a onto 2-chlorotrityl chloride resin and coupling $\mathbf{4 b}, \mathbf{4 c}, \mathbf{4 d}$, and $\mathbf{4 e}$ in succession (35 $\mathrm{mg}, 96 \%$ ). H-Mmb5-4-3-2-1-OH (26 mg, $22 \mu \mathrm{mol}$ ) was cyclized according to general procedure (e) and the residue was purified by precipitation from cold water and collected by centrifugation and purified by flash chromatography 
(methanol/methylene chloride) to give the title compound as a white powder (17 mg, 64\% cyclization, $62 \%$ overall from resin loading). ${ }^{1} \mathrm{H}$ NMR $\left(600 \mathrm{MHz}, \mathrm{DMSO}-d_{6}\right) \delta 8.43(\mathrm{t}, J=6.2 \mathrm{~Hz}, 1 \mathrm{H}), 8.41-8.33(\mathrm{~m}, 4 \mathrm{H})$, $7.70-7.67(\mathrm{~m}, 5 \mathrm{H}), 6.73(\mathrm{~s}, 1 \mathrm{H}), 6.72(\mathrm{~s}, 2 \mathrm{H}), 6.69(\mathrm{~s}, 1 \mathrm{H}), 6.68(\mathrm{~s}, 1 \mathrm{H}), 4.38-4.32(\mathrm{~m}, 10 \mathrm{H}), 4.22-4.14(\mathrm{~m}$, $6 \mathrm{H}), 3.96-3.92(\mathrm{~m}, 21 \mathrm{H}), 1.90-1.63(\mathrm{~m}, 11 \mathrm{H}), 1.41(\mathrm{t}, J=7.0 \mathrm{~Hz}, 3 \mathrm{H}), 1.34-1.08(\mathrm{~m}, 5 \mathrm{H}), 1.01(\mathrm{~s}, 9 \mathrm{H})$, $0.97(\mathrm{~d}, J=6.6 \mathrm{~Hz}, 6 \mathrm{H}) .{ }^{13} \mathrm{C}$ NMR $\left(101 \mathrm{MHz}, \mathrm{DMSO}-d_{6}\right) \delta 164.71,164.68,164.54,160.42,159.80,159.77$, $159.68,158.38,158.35,158.28,130.68,130.58,130.52,120.01,119.96,119.89,119.85,113.82,113.68$, $113.63,113.56,96.85,96.80,96.76,96.25,73.59,67.00,66.05,64.28,56.89,56.84,56.81,56.36,37.96,37.85$, 30.08, 29.76, 26.52, 25.82, 25.20, 22.99, 15.06. MS (MALDI-TOF ${ }^{+}$) Calcd for $\mathrm{C}_{66} \mathrm{H}_{85} \mathrm{~N}_{5} \mathrm{O}_{15} \mathrm{Na}^{+}[\mathrm{M}+\mathrm{Na}]^{+}$ 1210.6 , found 1210.4 .

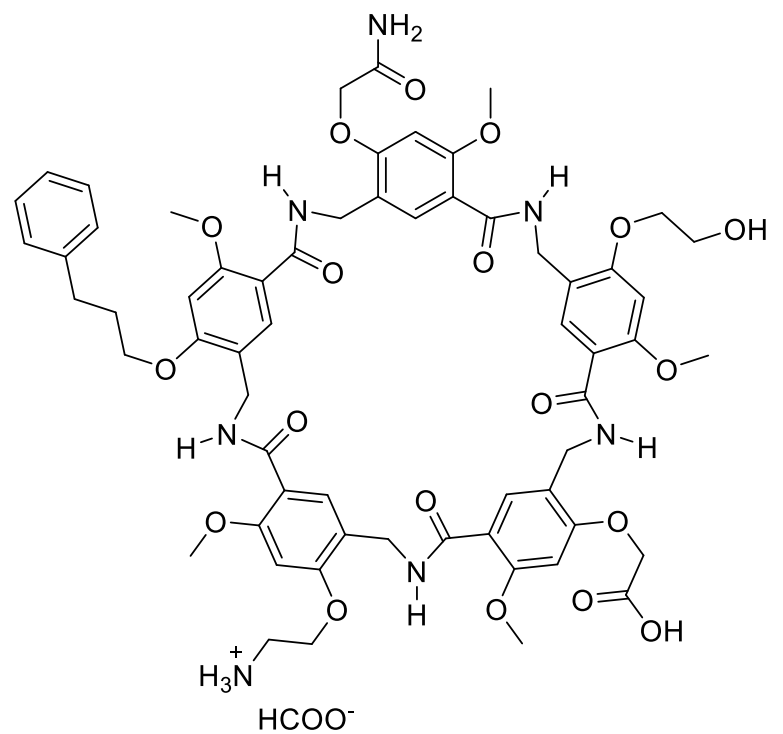

cyclo(Mmb8-7-10-9-6)・HCOOH

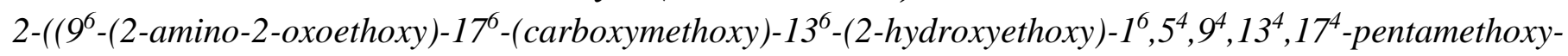
2,6,10,14,18-pentaoxo-56-(3-phenylpropoxy)-3,7,11,15,19-pentaaza-1,5,9,13,17(1,3)-

pentabenzenacycloicosaphane-14-yl)oxy)ethan-1-aminium formate

According to general procedure (d): $\mathrm{H}-\mathrm{Mmb} 8(\mathrm{Tr})-7(\mathrm{OtBu})-10(\mathrm{OtBu})-9(\mathrm{Boc})-6-\mathrm{OH}$ was prepared on a $30 \mu \mathrm{mol}$ scale by loading Fmoc-Mmb6-OH, $\mathbf{4 f}$ onto 2-chlorotrityl chloride resin and coupling $\mathbf{4 i}, \mathbf{4 j}$, and $\mathbf{4 g}$, and $\mathbf{4 h}$ in succession (50 mg, 98\%). H- Mmb8(Tr)-7(OtBu)-10(OtBu)-9(Boc)-6-OH (30 mg, $18 \mu \mathrm{mol}$ ) was cyclized according to general procedure (e) and the residue was purified by precipitation from cold water and collected by centrifugation and dried in vacuo. The crude material was deprotected according to general procedure (f), concentrated in vасиo, and purified by UHPLC (methanol/water/acetonitrile) to give the title compound (formate salt) as a colorless solid (10 mg, 44\% cyclization, 43\% overall from resin loading). ${ }^{1} \mathrm{H}$ NMR (600 MHz, DMSO-d $) \delta 8.56(\mathrm{t}, J=6.5 \mathrm{~Hz}, 1 \mathrm{H}), 8.46(\mathrm{t}, J=6.6 \mathrm{~Hz}, 1 \mathrm{H}), 8.42(\mathrm{q}, J=6.4 \mathrm{~Hz}, 3 \mathrm{H}), 8.04(\mathrm{dt}, J=12.1$, $6.6 \mathrm{~Hz}, 4 \mathrm{H}), 7.81(\mathrm{~s}, 1 \mathrm{H}), 7.74(\mathrm{~s}, 2 \mathrm{H}), 7.73(\mathrm{~s}, 1 \mathrm{H}), 7.67(\mathrm{~s}, 1 \mathrm{H}), 7.61(\mathrm{~s}, 1 \mathrm{H}), 7.57(\mathrm{~s}, 1 \mathrm{H}), 7.53(\mathrm{~s}, 1 \mathrm{H}), 7.28$ $(\mathrm{dt}, J=15.0,7.5 \mathrm{~Hz}, 5 \mathrm{H}), 7.19(\mathrm{t}, J=6.9 \mathrm{~Hz}, 1 \mathrm{H}), 6.72(\mathrm{~s}, 1 \mathrm{H}), 6.70(\mathrm{~s}, 1 \mathrm{H}), 6.69(\mathrm{~s}, 1 \mathrm{H}), 6.68(\mathrm{~s}, 1 \mathrm{H}), 6.66(\mathrm{~s}$, $1 \mathrm{H}), 4.90(\mathrm{~s}, 2 \mathrm{H}), 4.63(\mathrm{~s}, 2 \mathrm{H}), 4.48-4.37(\mathrm{~m}, 10 \mathrm{H}), 4.34-4.30(\mathrm{~m}, 2 \mathrm{H}), 4.15(\mathrm{q}, J=6.5,5.7 \mathrm{~Hz}, 6 \mathrm{H}), 3.95-$ $3.87(\mathrm{~m}, 15 \mathrm{H}), 3.84-3.77(\mathrm{~m}, 4 \mathrm{H}), 3.32-3.27(\mathrm{~m}, 4 \mathrm{H}), 2.81(\mathrm{t}, J=7.7 \mathrm{~Hz}, 2 \mathrm{H}), 2.13-2.07(\mathrm{~m}, 2 \mathrm{H}), 1.96-$ $1.87(\mathrm{~m}, 2 \mathrm{H}) .{ }^{13} \mathrm{C}$ NMR $\left(151 \mathrm{MHz}, \mathrm{DMSO}-d_{6}\right) \delta 170.04,169.38,164.72,164.28,164.05,163.98,163.91$, 159.32, 158.93, 158.44, 158.12, 157.70, 157.63, 157.56, 157.47, 157.44, 141.25, 131.42, 128.26, 128.25, $125.75,119.65,119.45,119.28,118.29,116.30,113.98,113.88,113.52,113.08,96.73,96.43,96.41,96.33$, $96.11,70.15,67.12,67.05,66.92,65.18,59.50,56.35,56.21,56.12,55.96,55.65,47.30,47.26,44.67,37.32$, 31.39, 30.33, 25.53, 23.55. MS (MALDI-TOF ${ }^{+}$) Calcd for $\mathrm{C}_{62} \mathrm{H}_{67} \mathrm{~N}_{7} \mathrm{O}_{19} \mathrm{Na}^{\cdot+}[\mathrm{M}+\mathrm{Na}]^{+}$1236.4, found 1236.7 . 
4. NMR Spectra

4.1. Small Molecule NMR Spectra

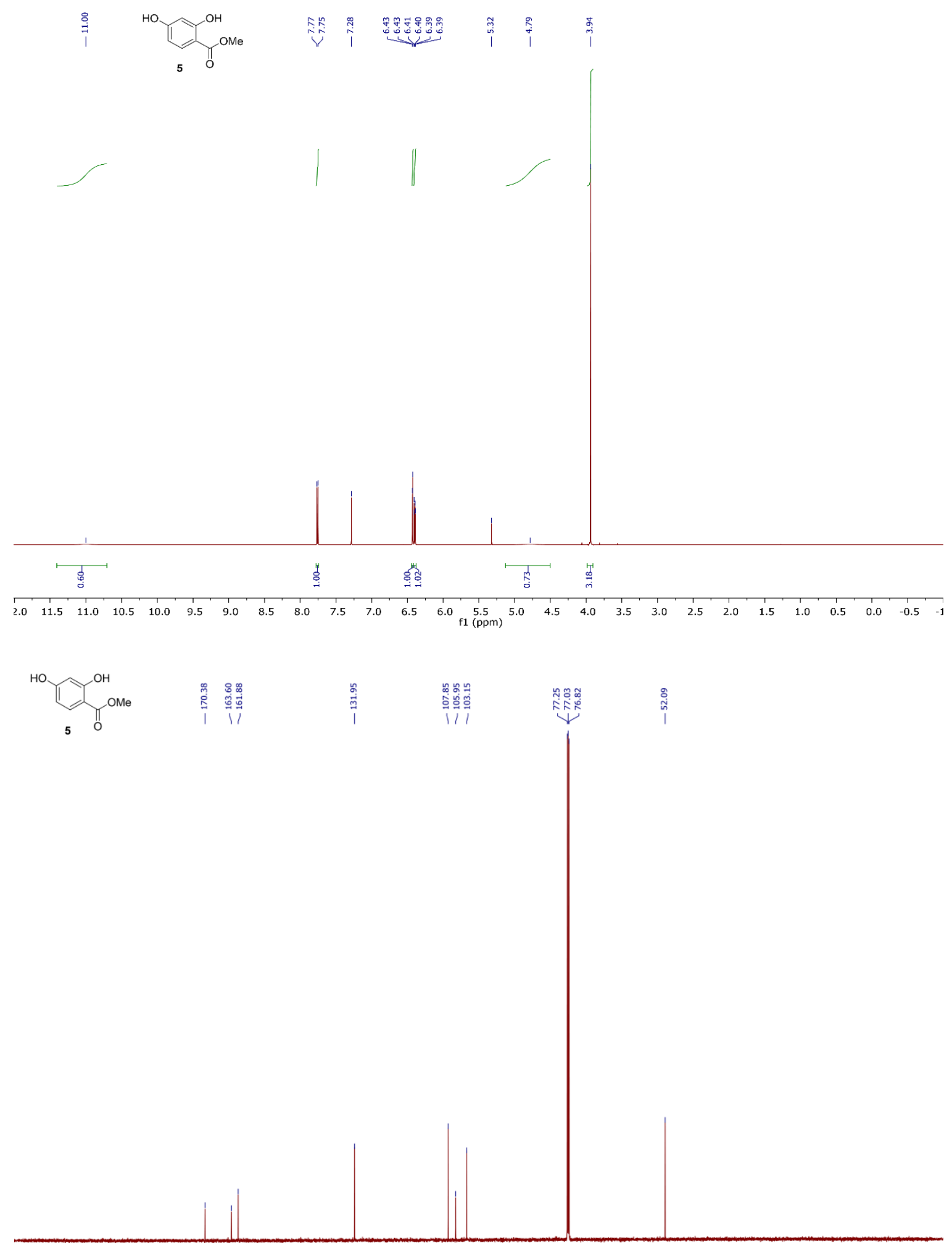

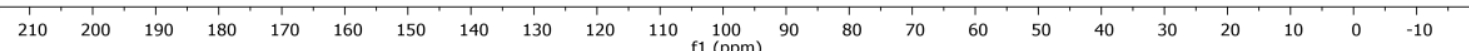



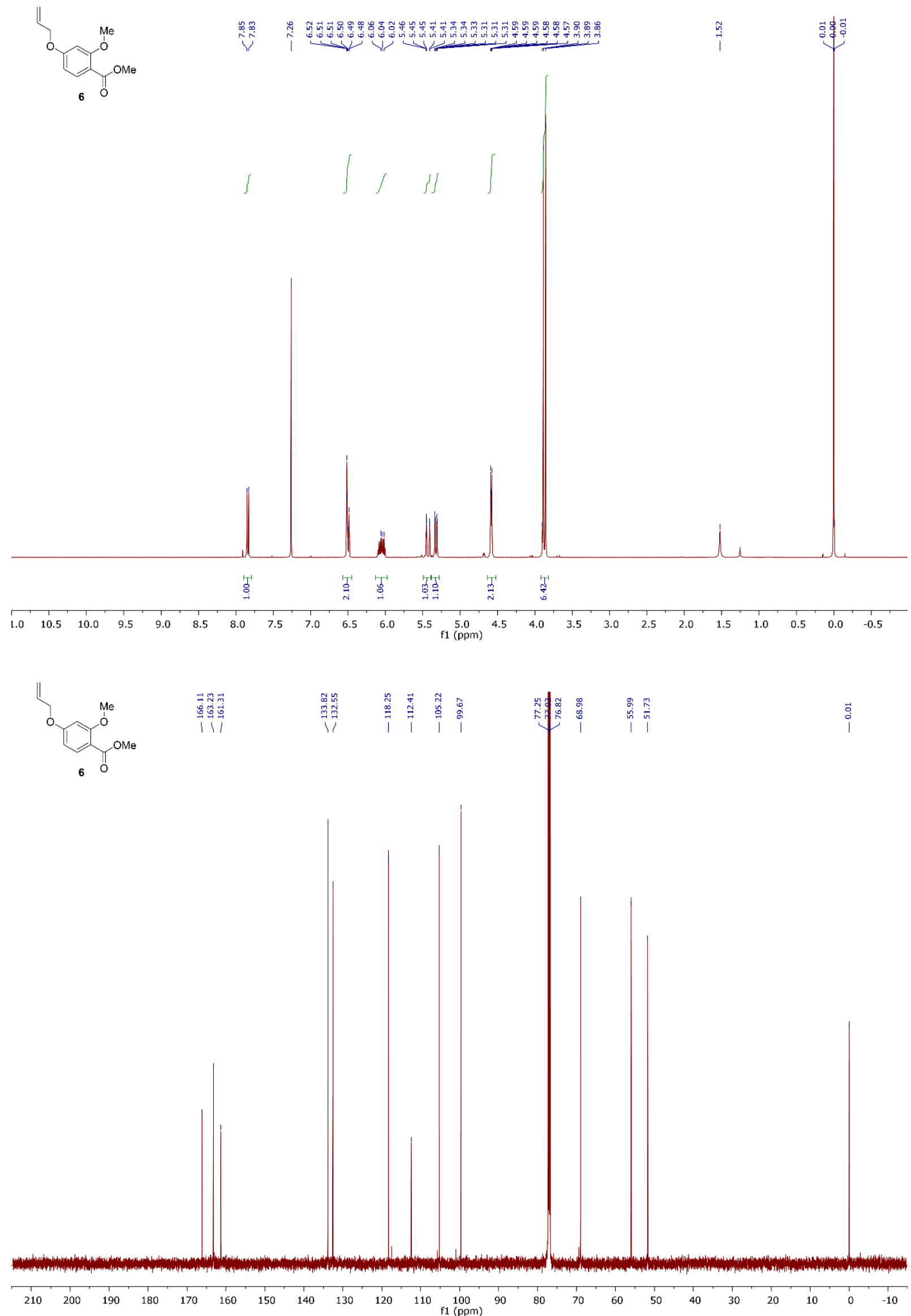


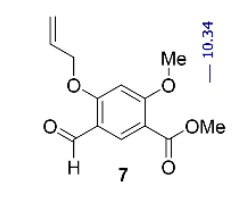

$\stackrel{\substack{\overrightarrow{0} \\ \infty}}{1}$

|

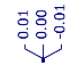
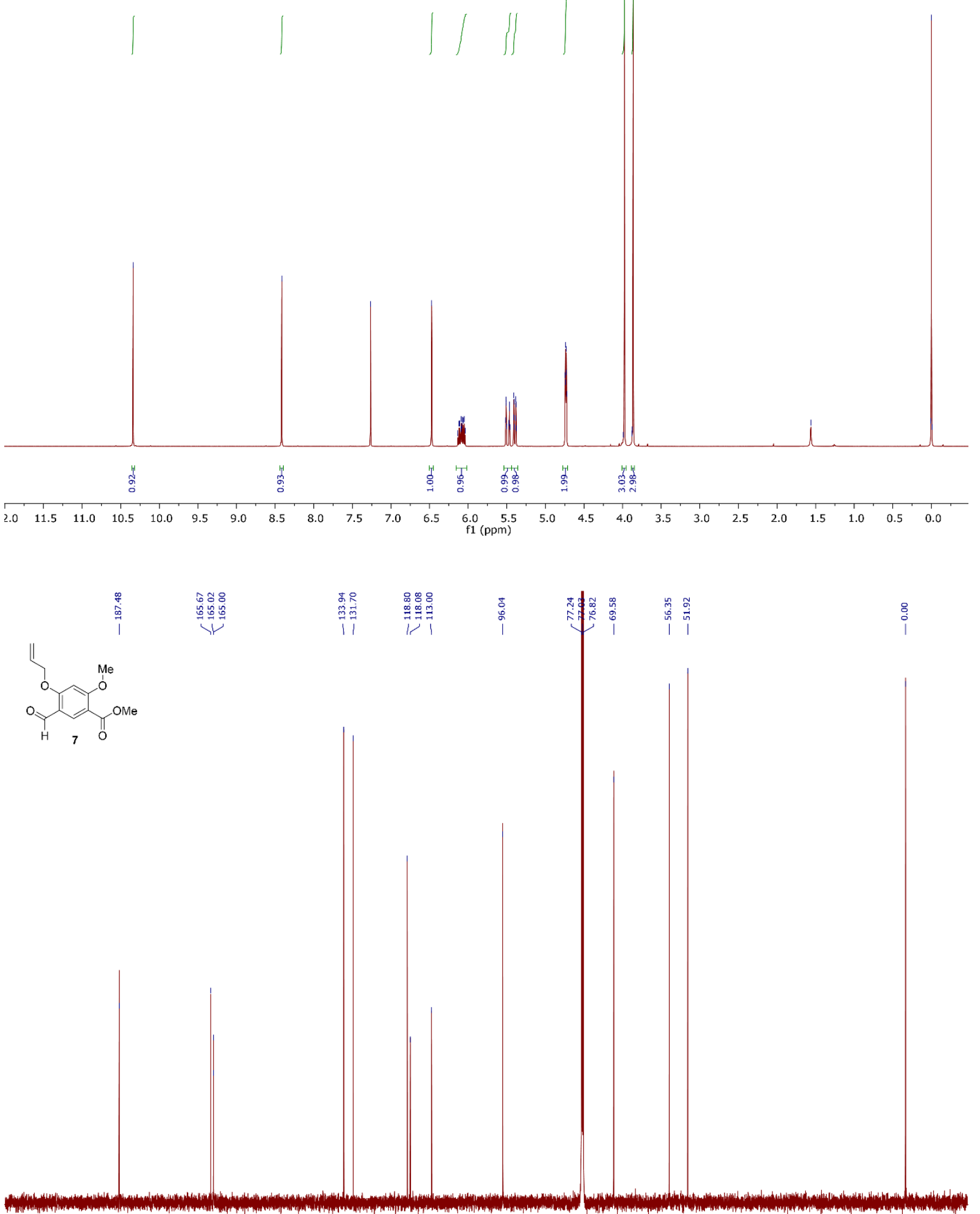

$\begin{array}{lllllllllllllllllllllllllllll}210 & 200 & 190 & 180 & 170 & 160 & 150 & 140 & 130 & 120 & 110 & 100 & 90 & 80 & 70 & 60 & 50 & 40 & 30 & 20 & 10 & 0 & -10\end{array}$ 

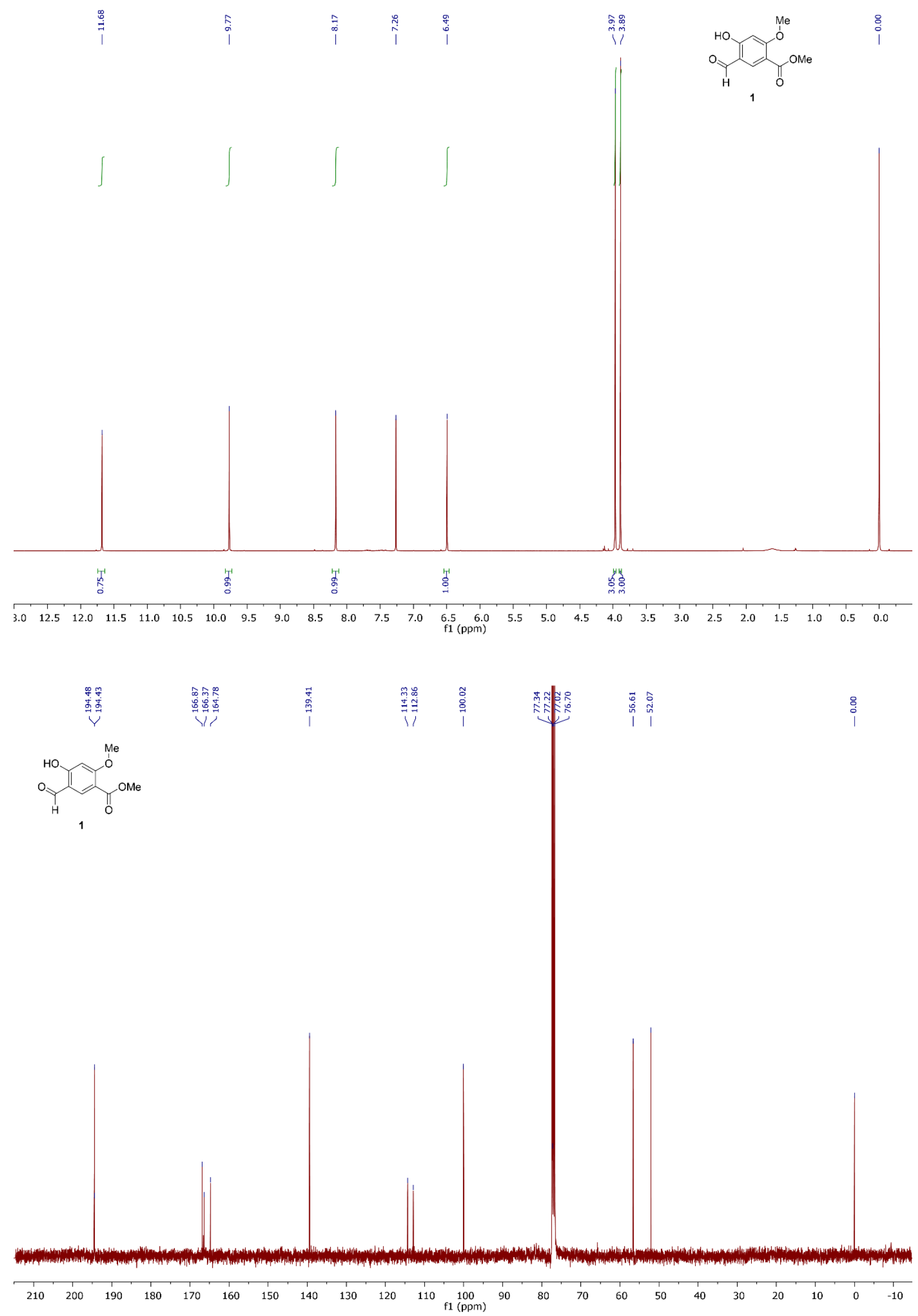

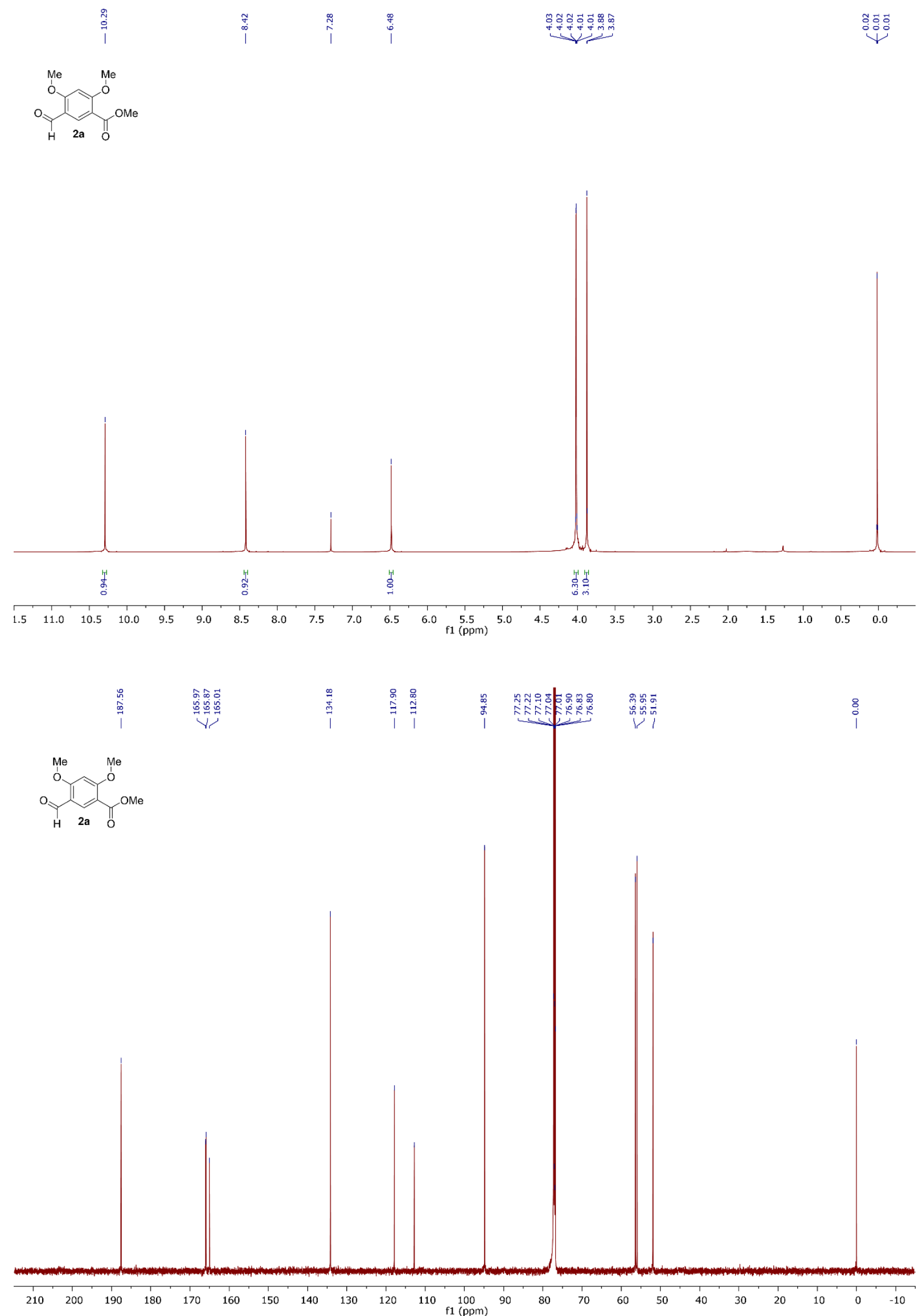
(2b

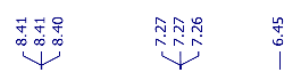

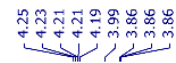

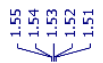

递

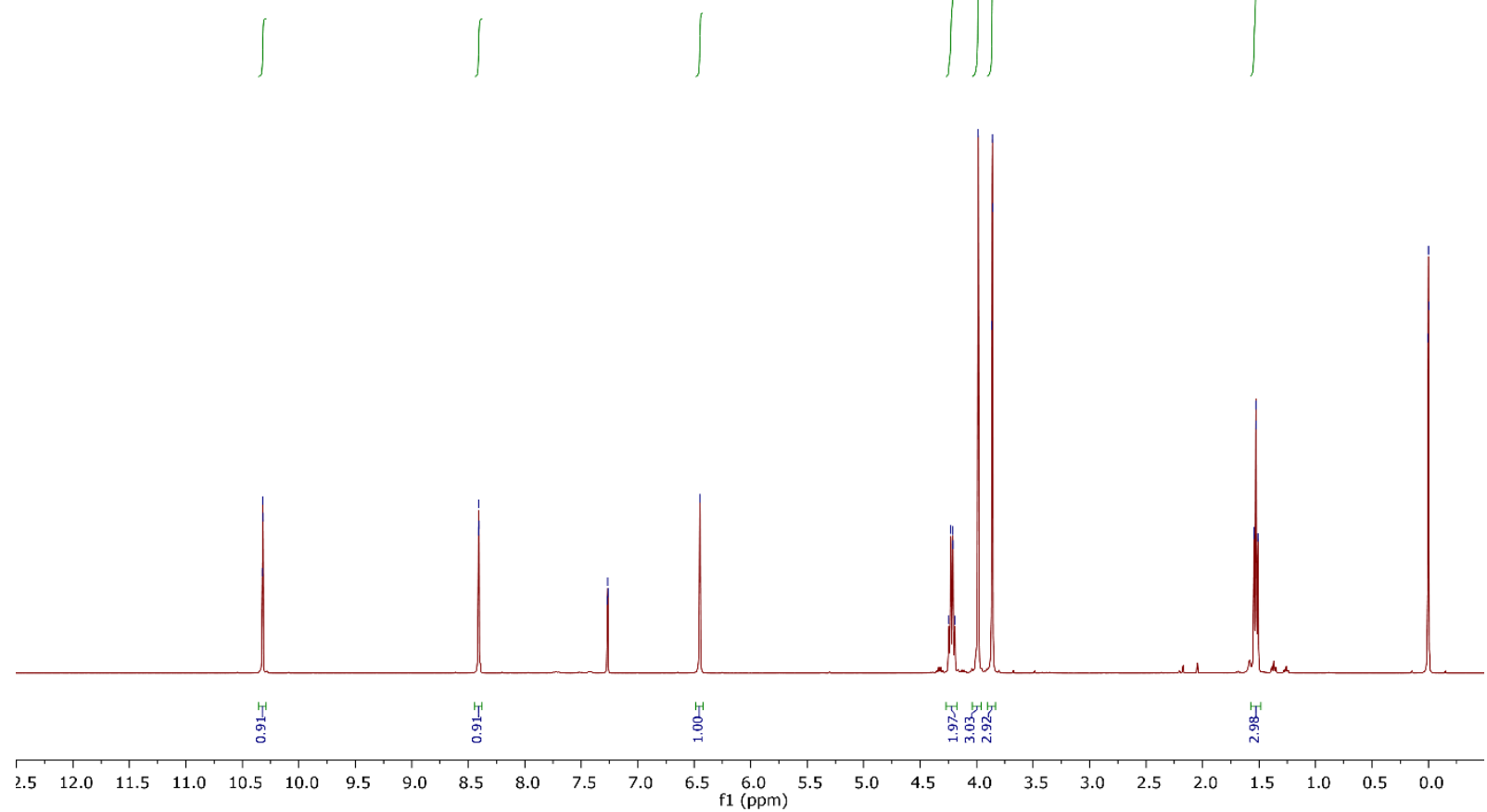

0
0

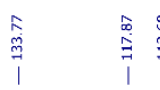

管

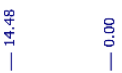
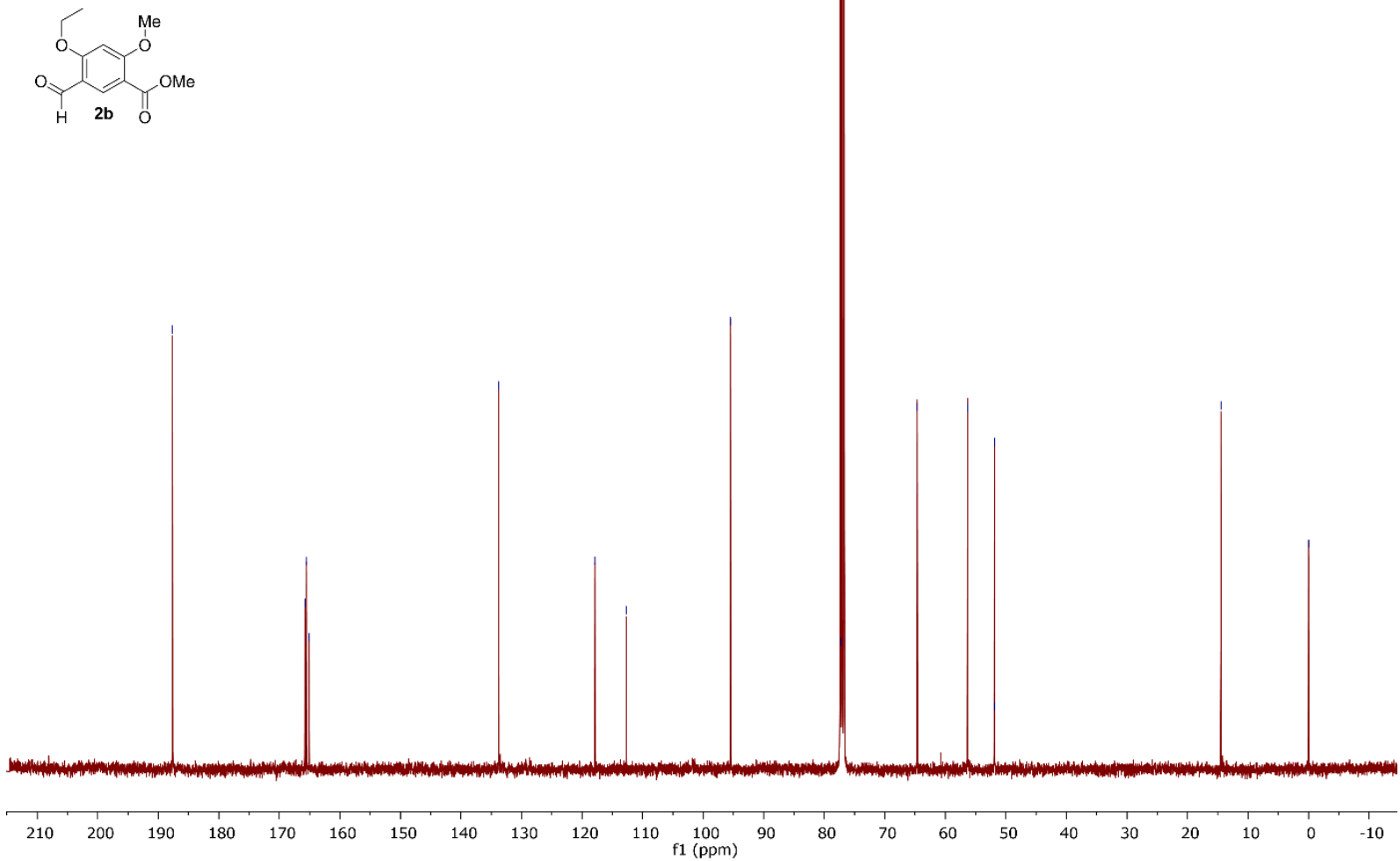

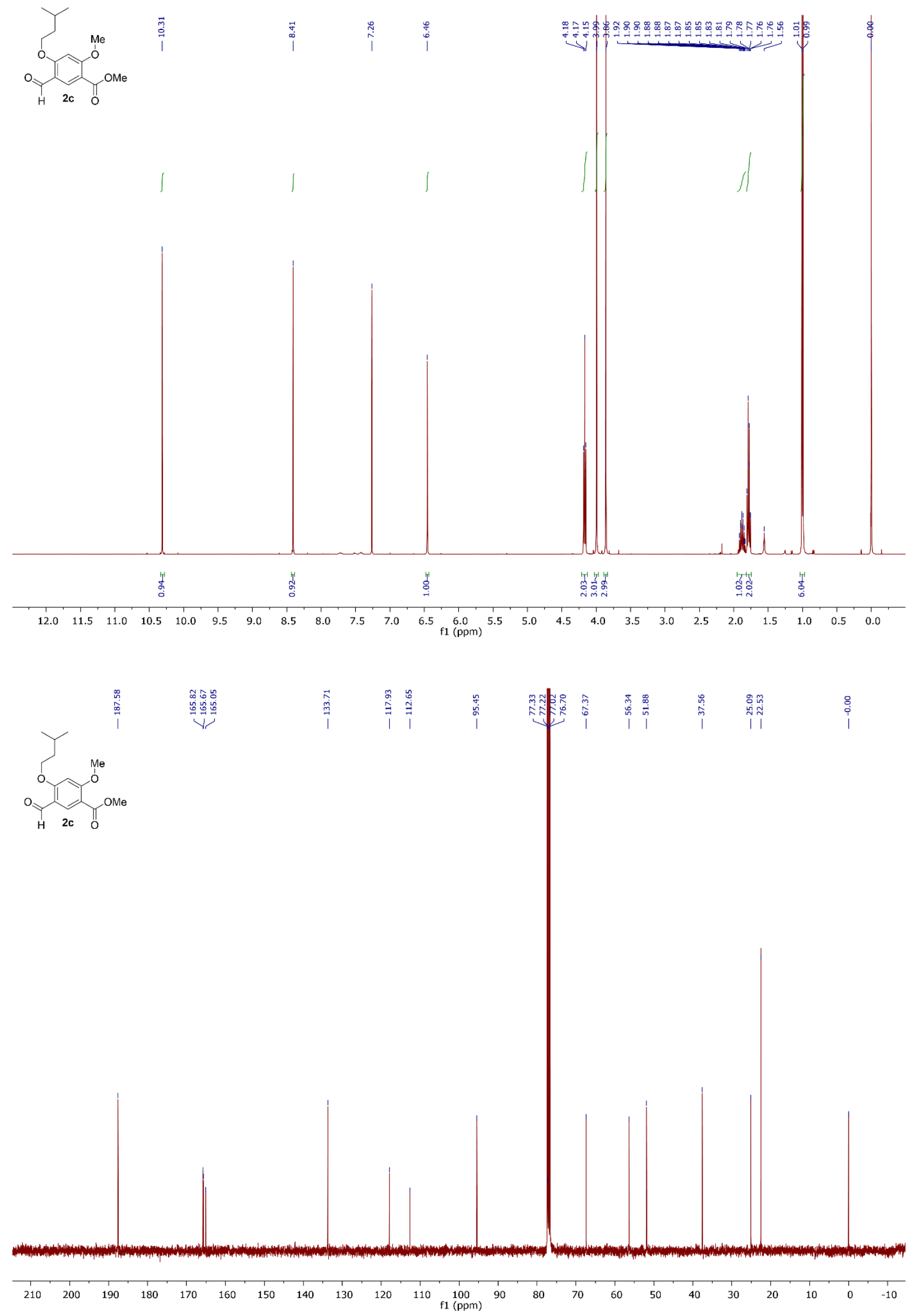

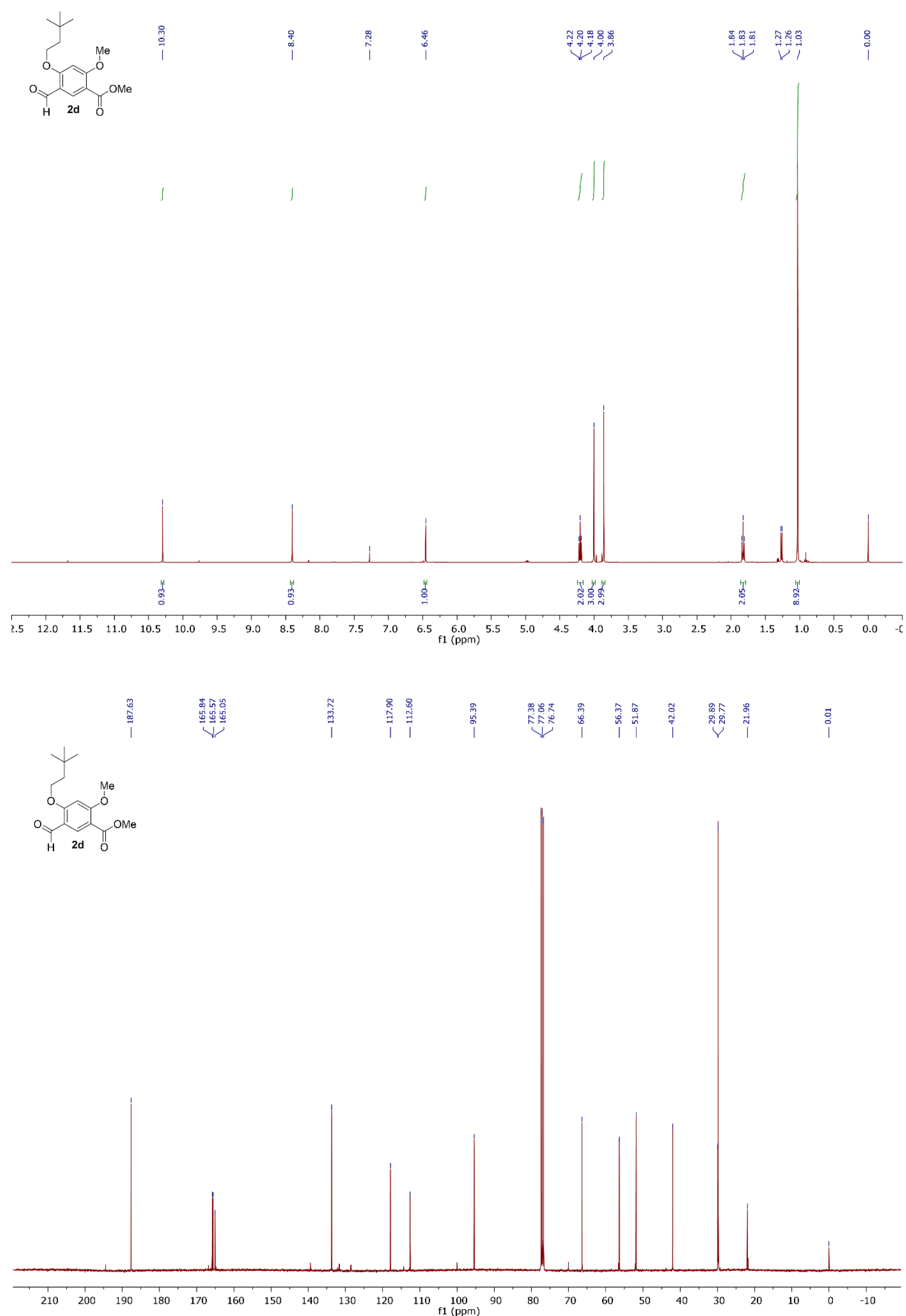

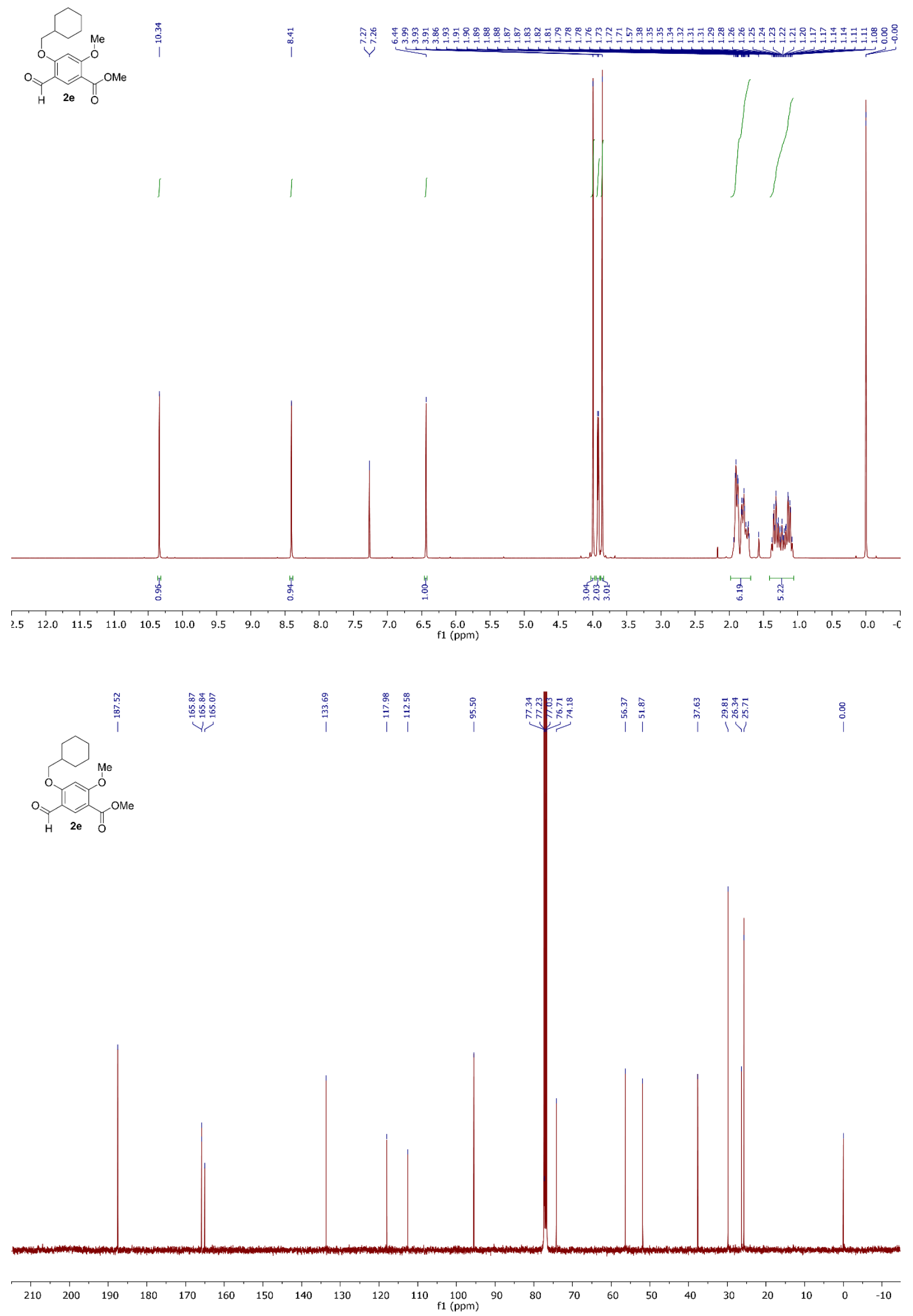

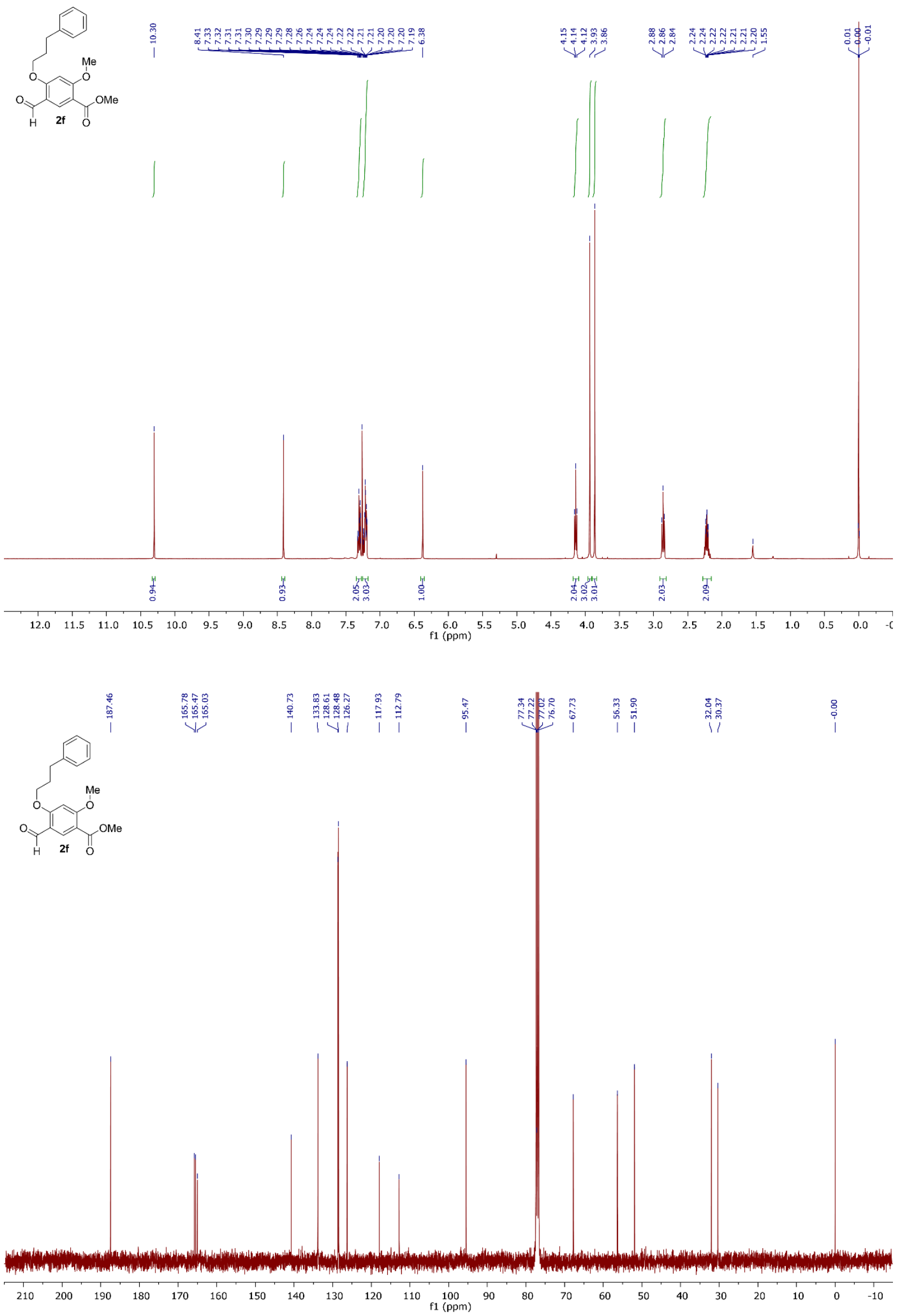

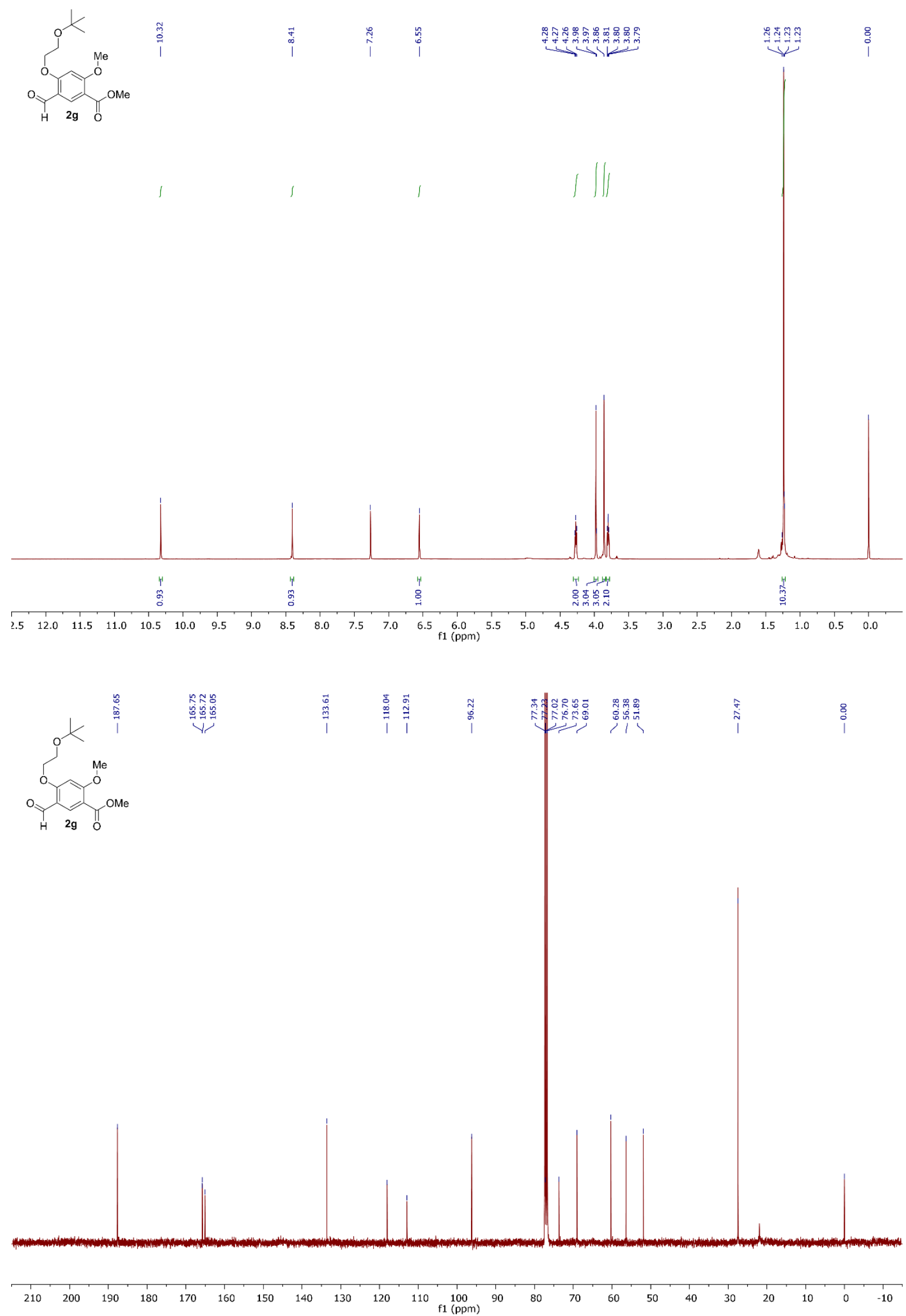

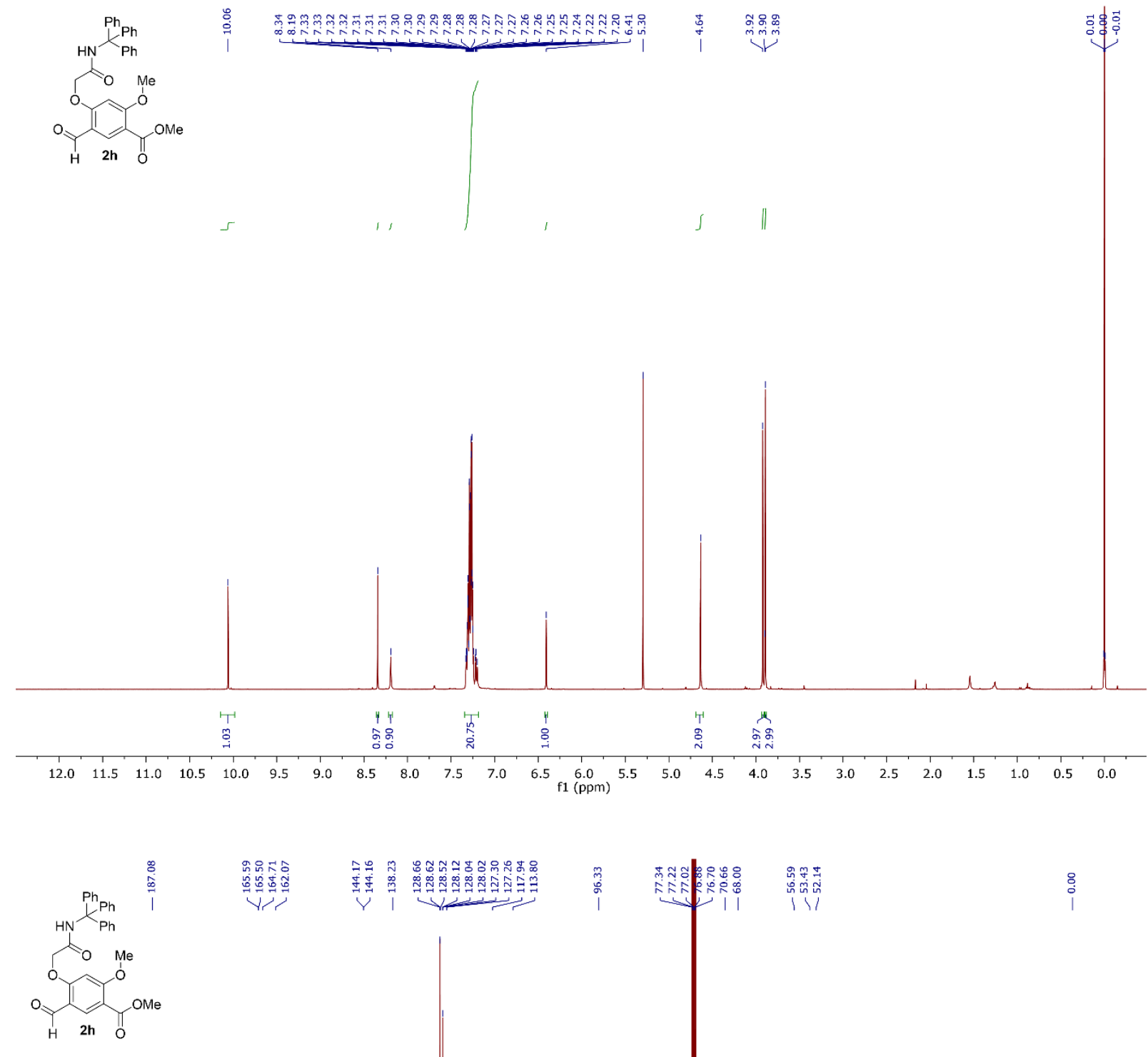

:

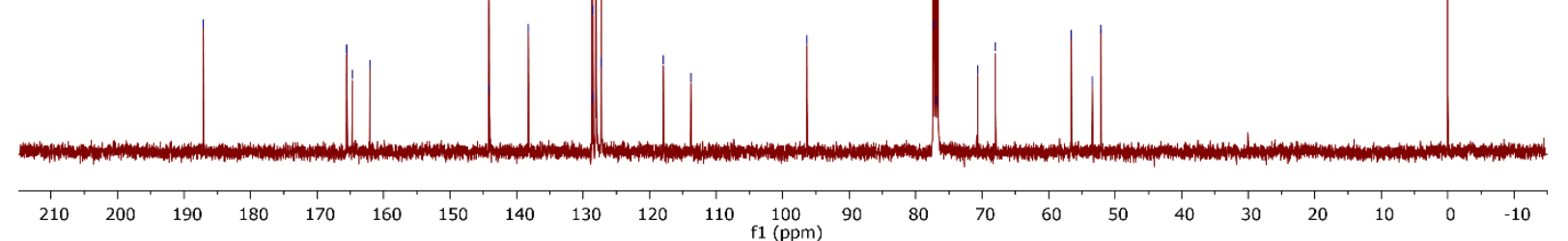



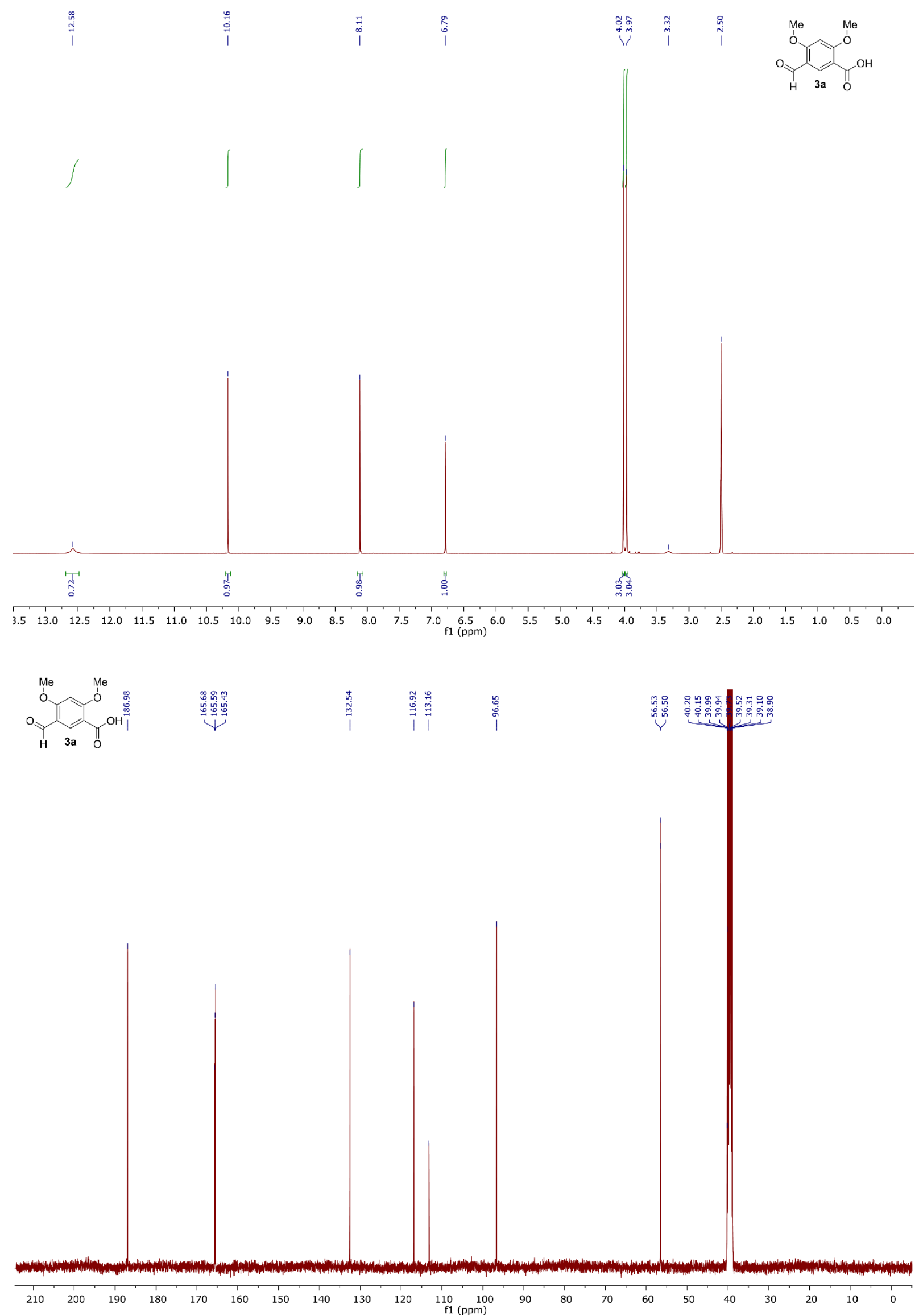

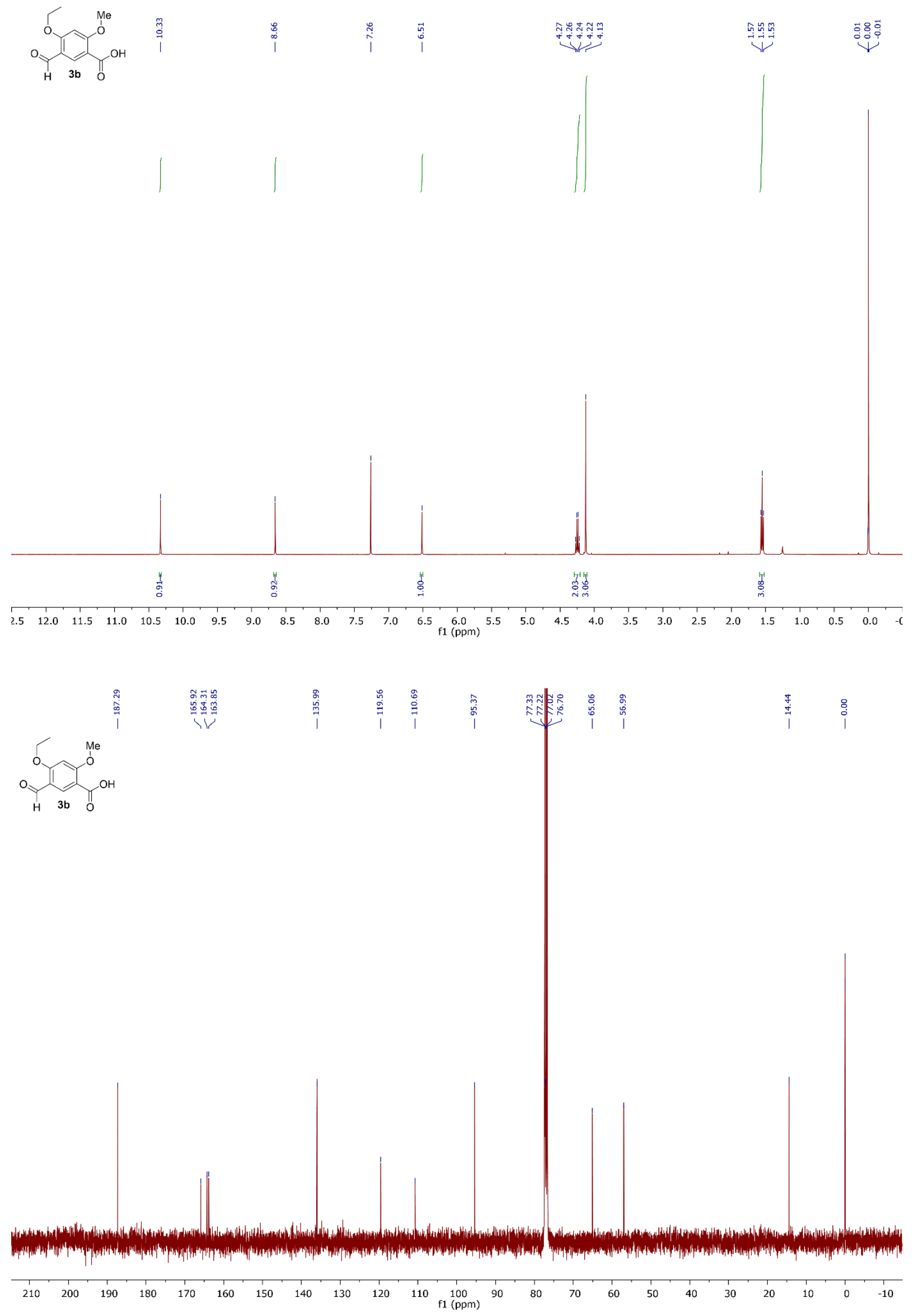

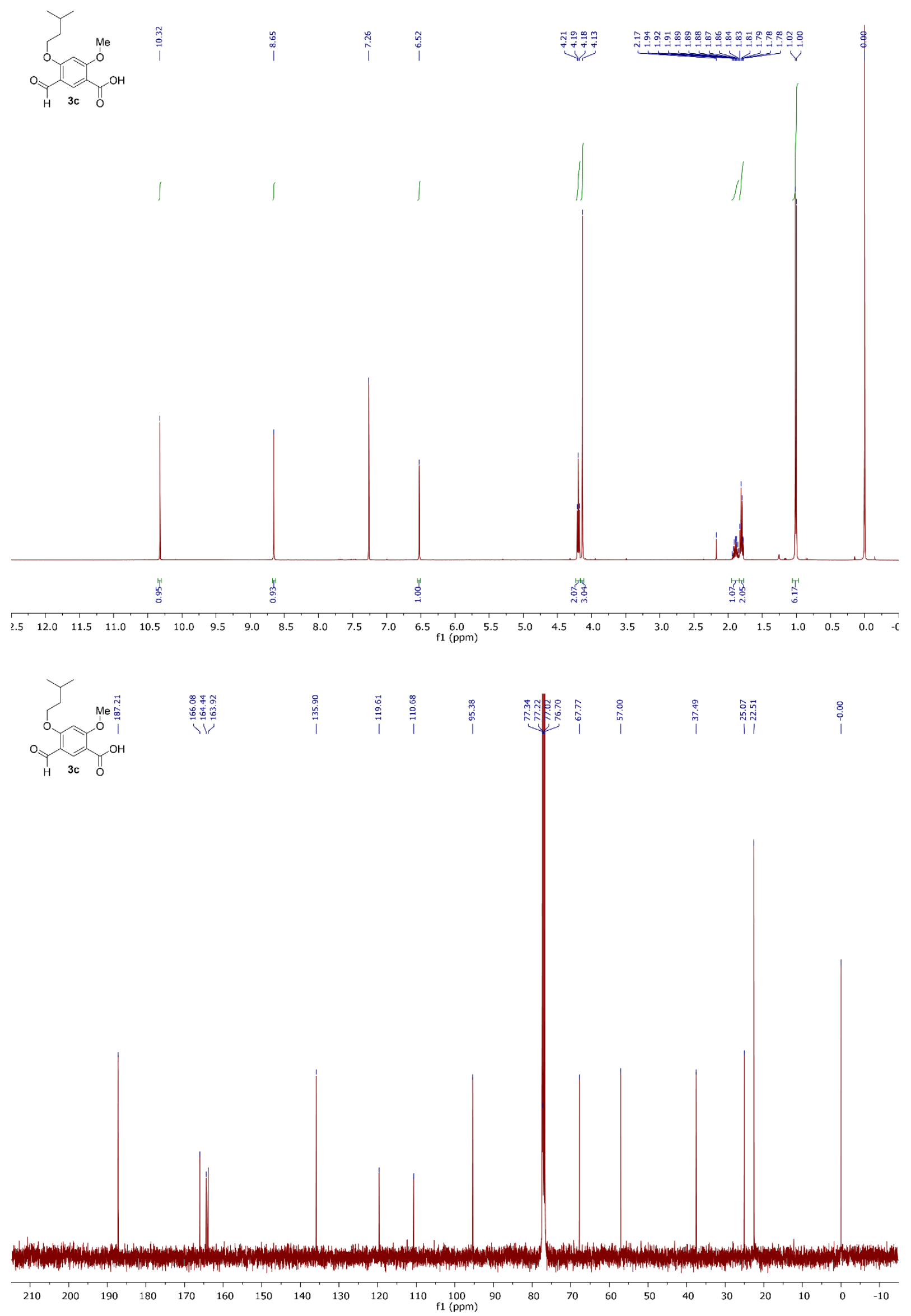

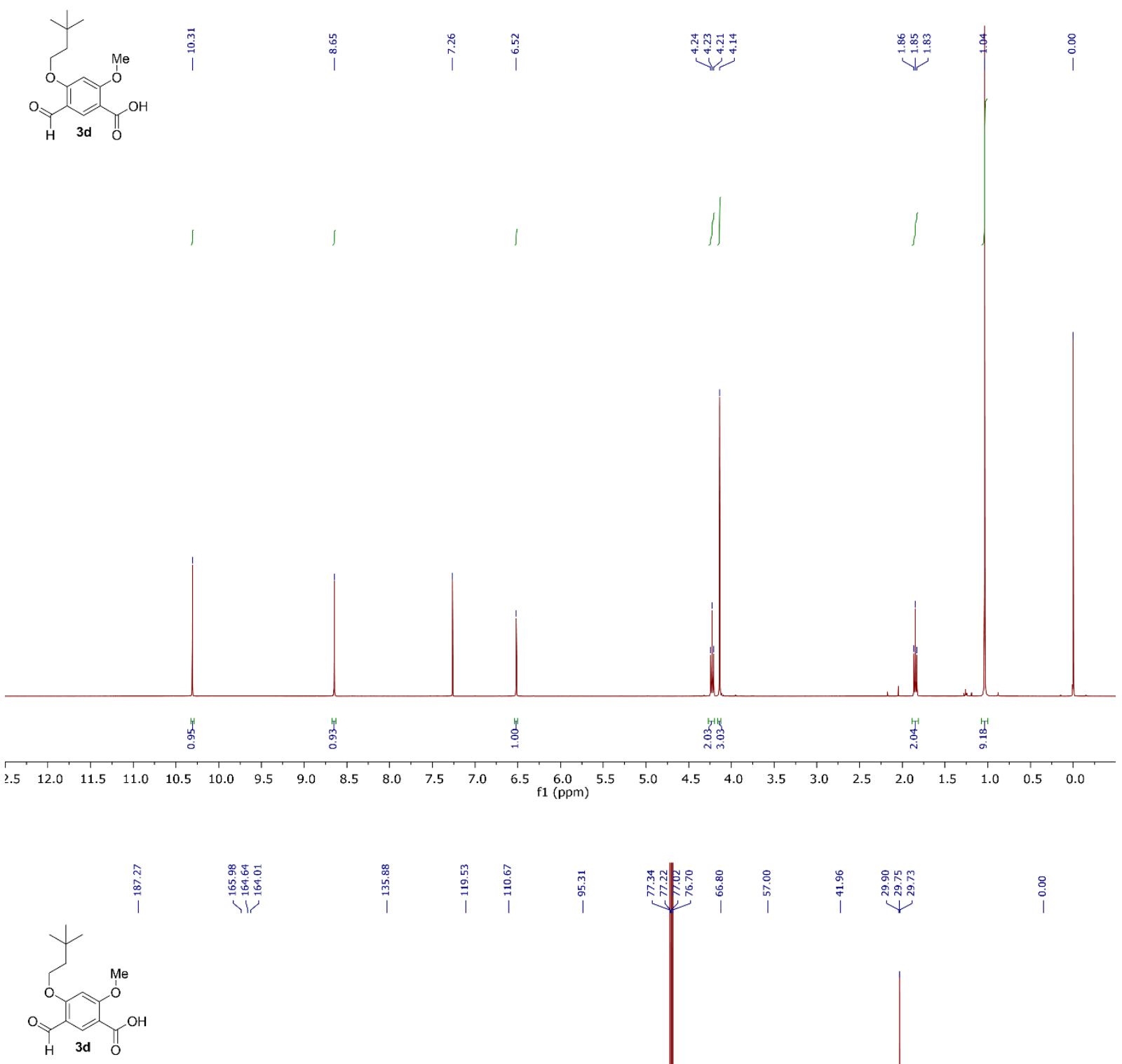

i

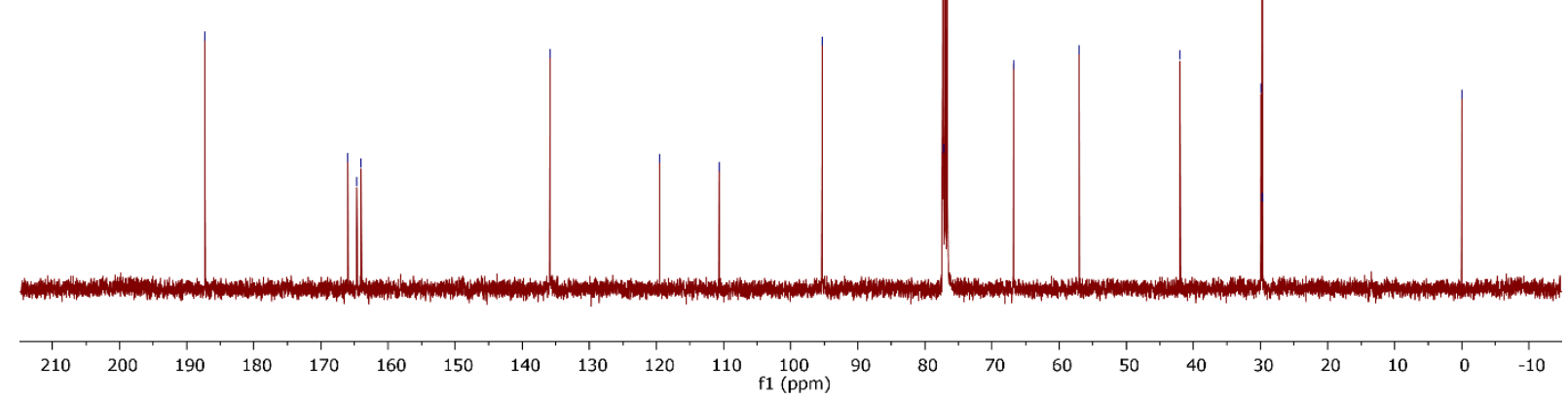



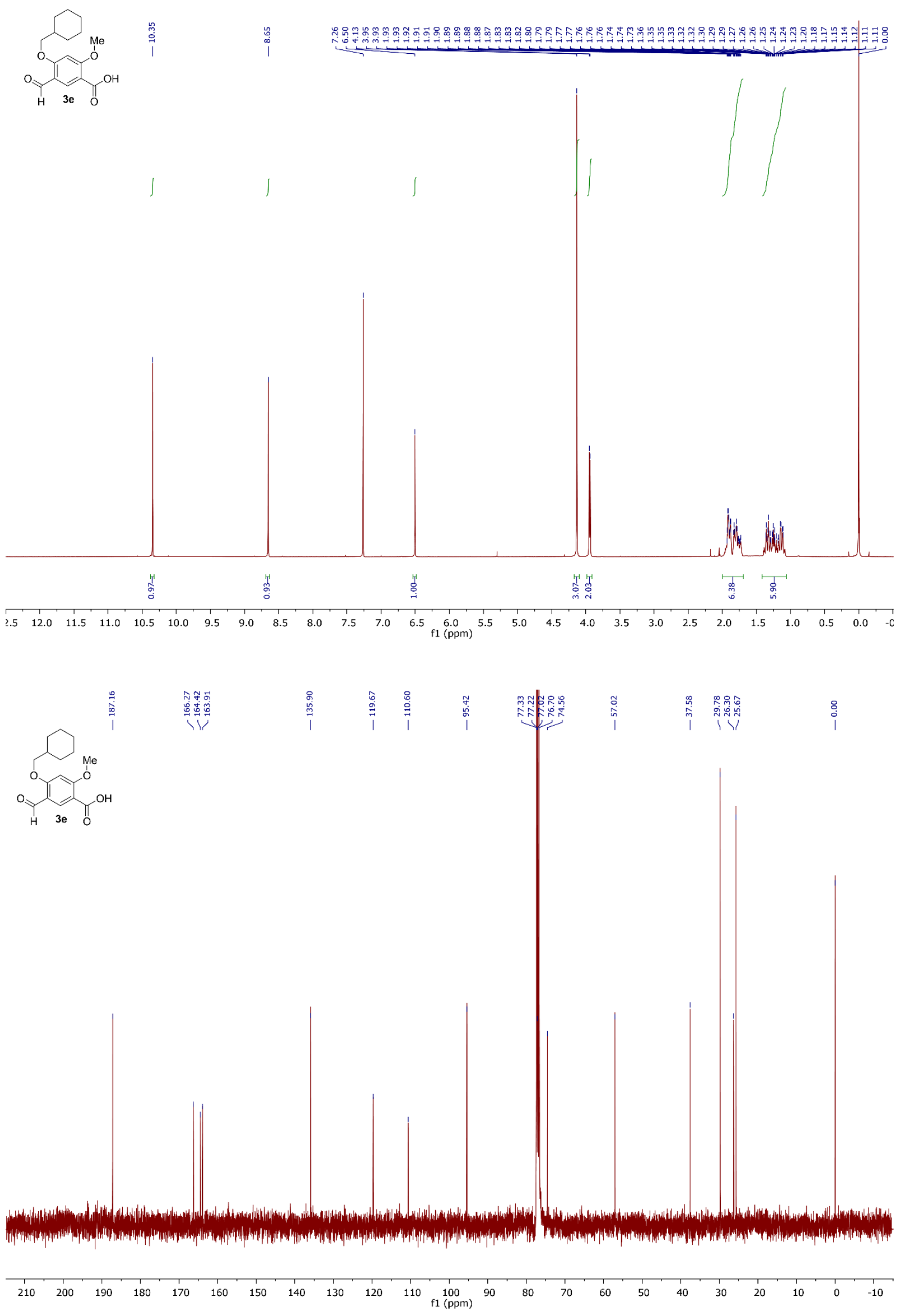

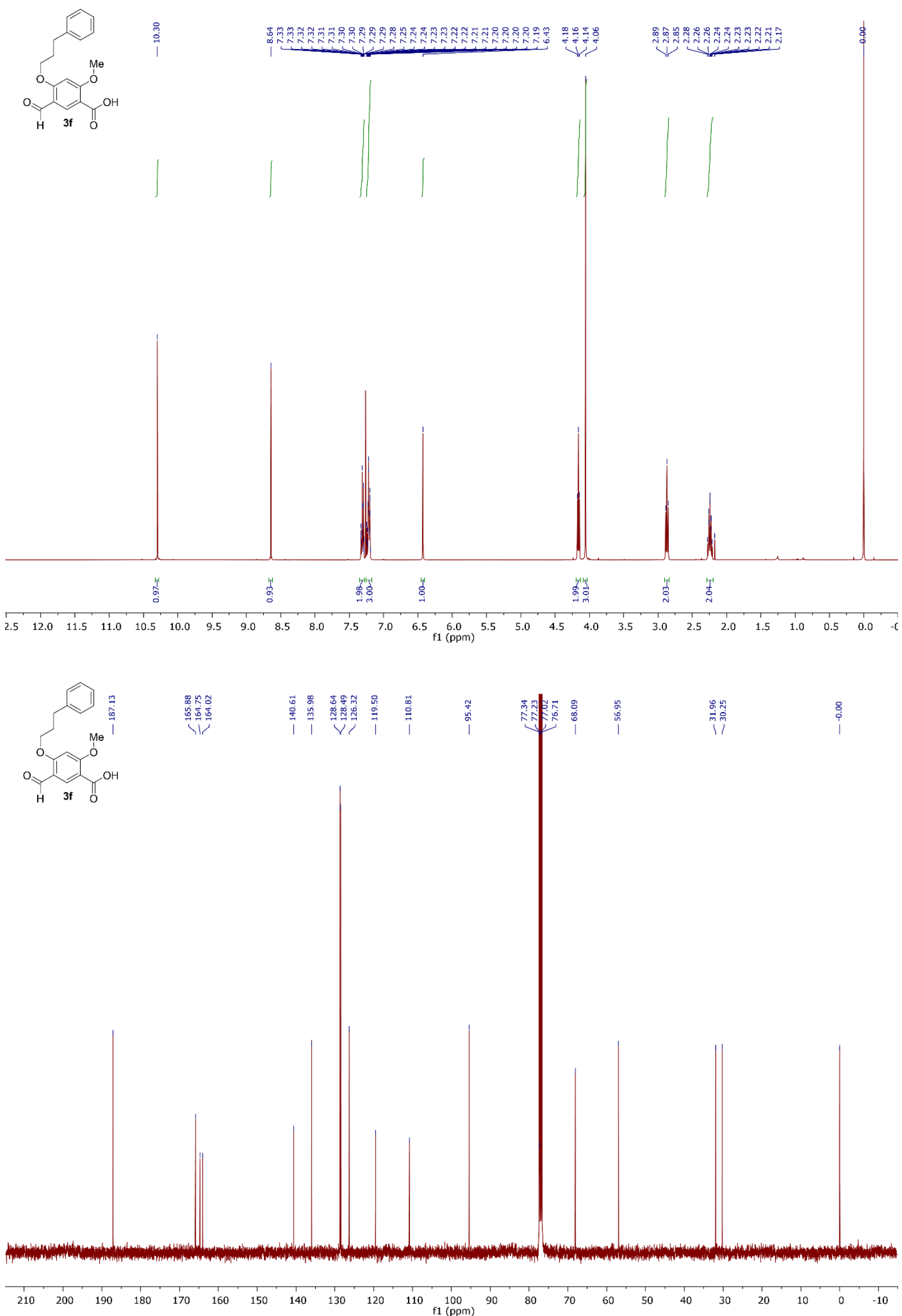

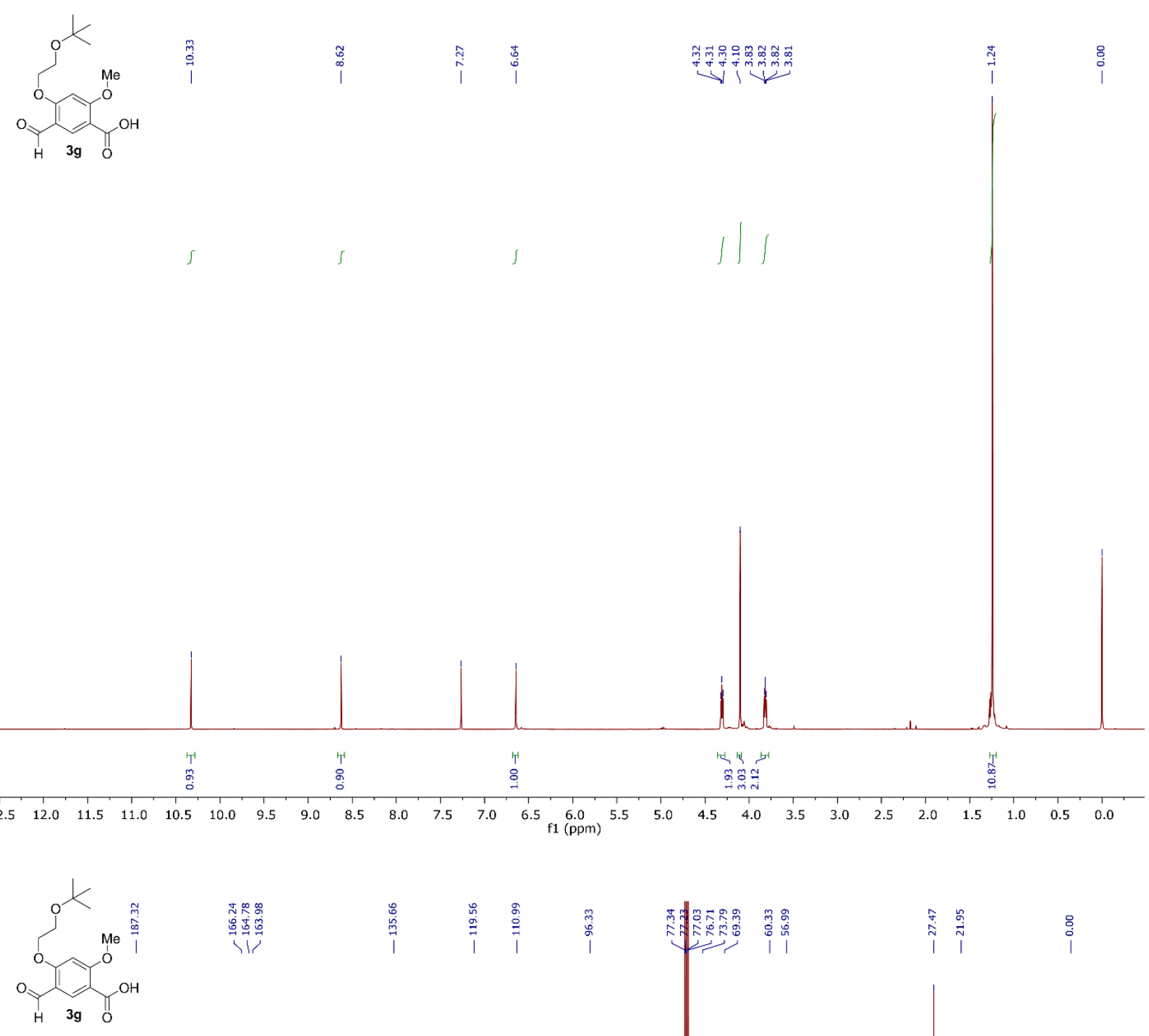

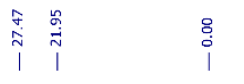



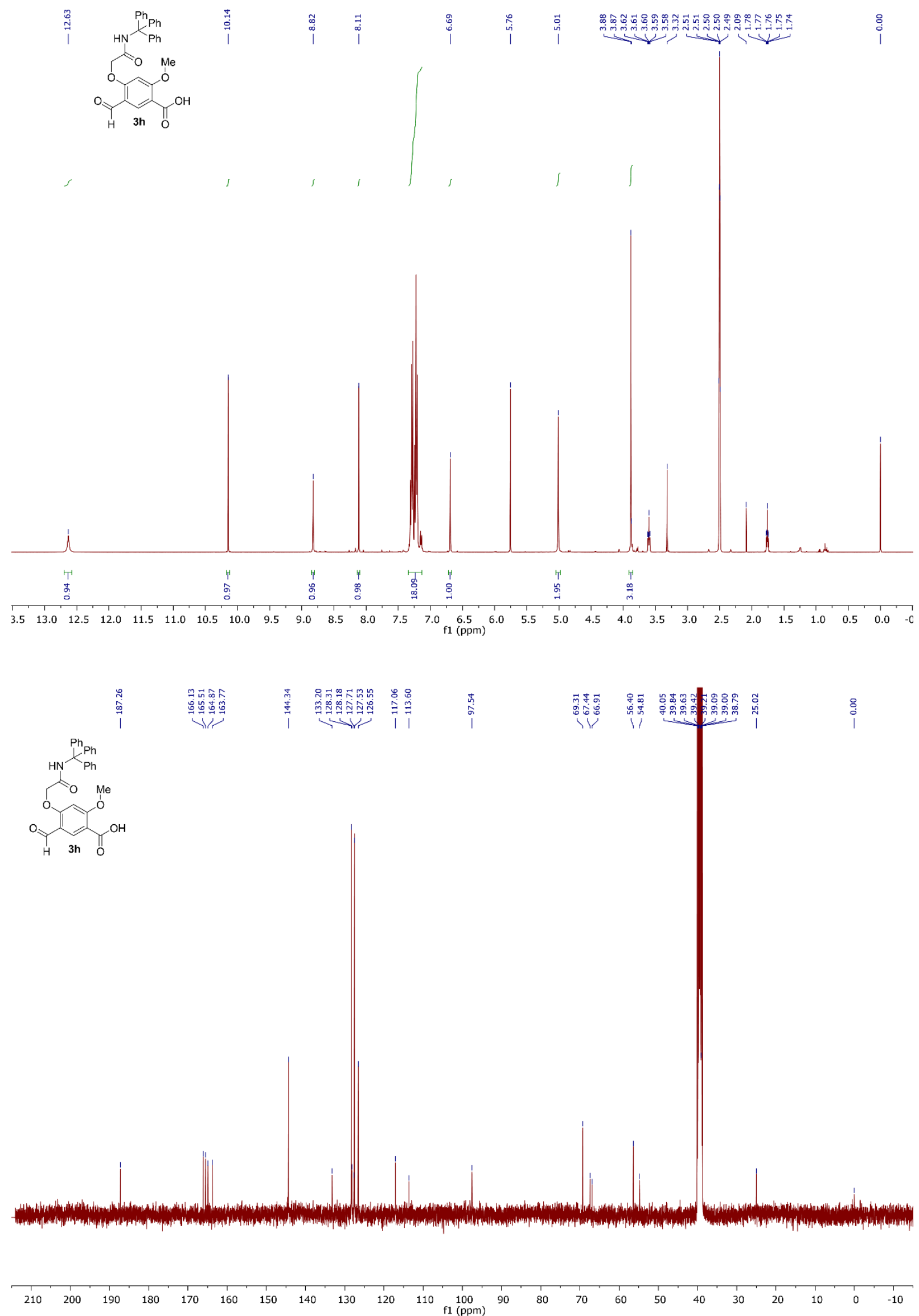

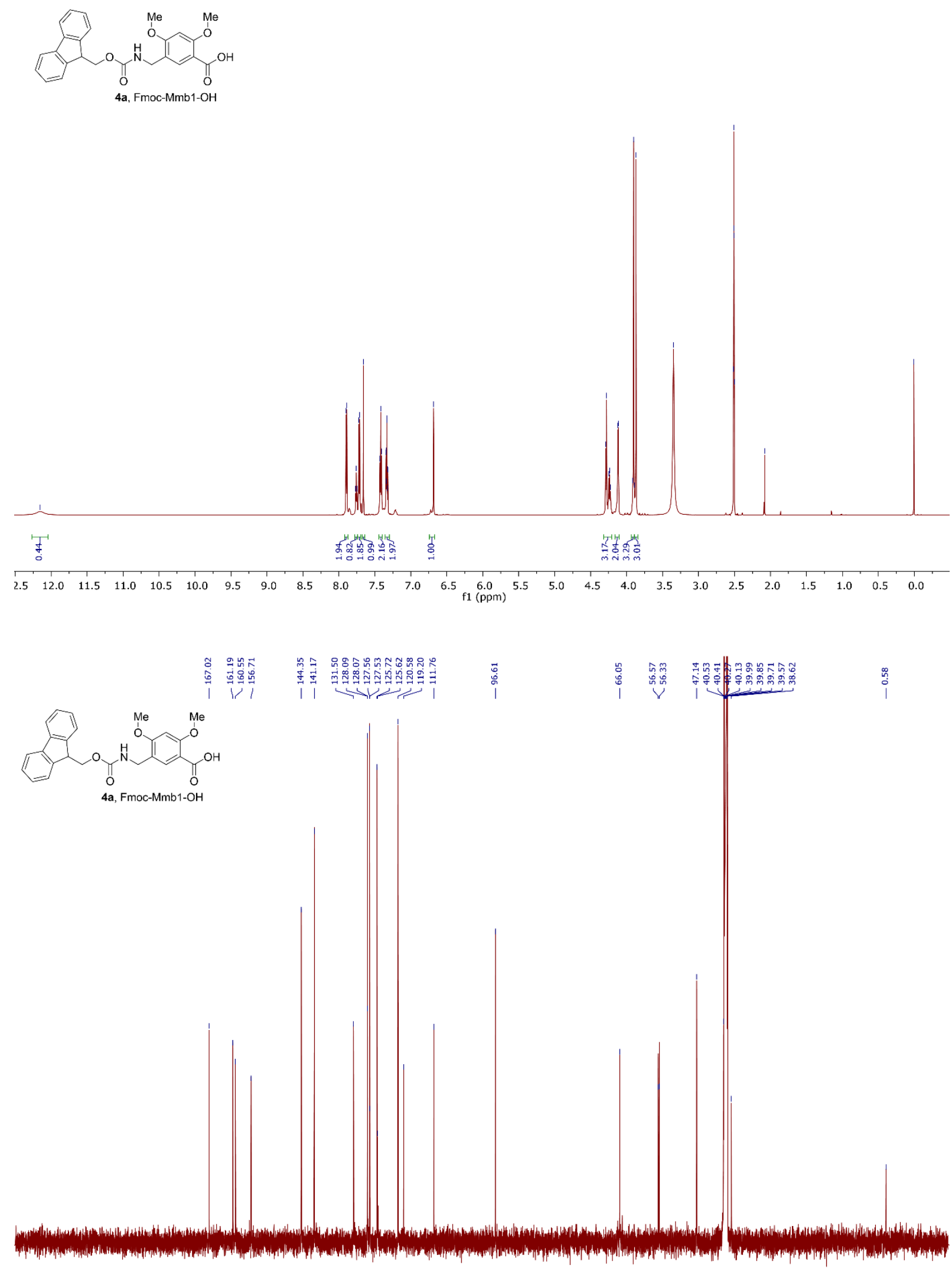

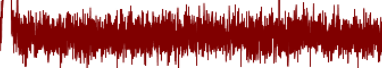

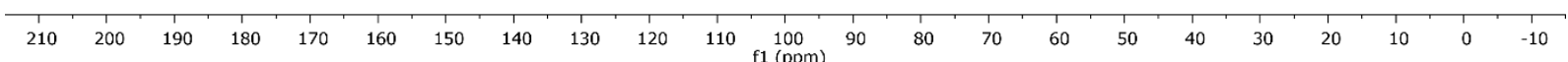




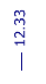
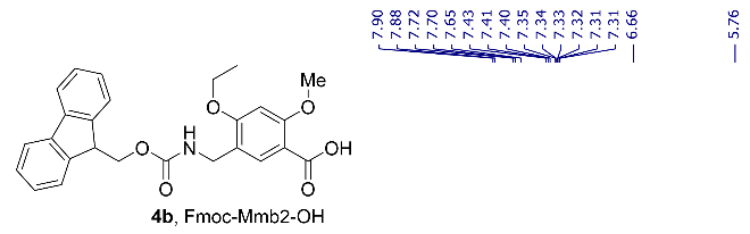

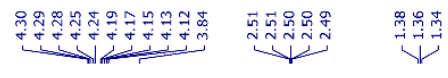

$\stackrel{8}{i}$

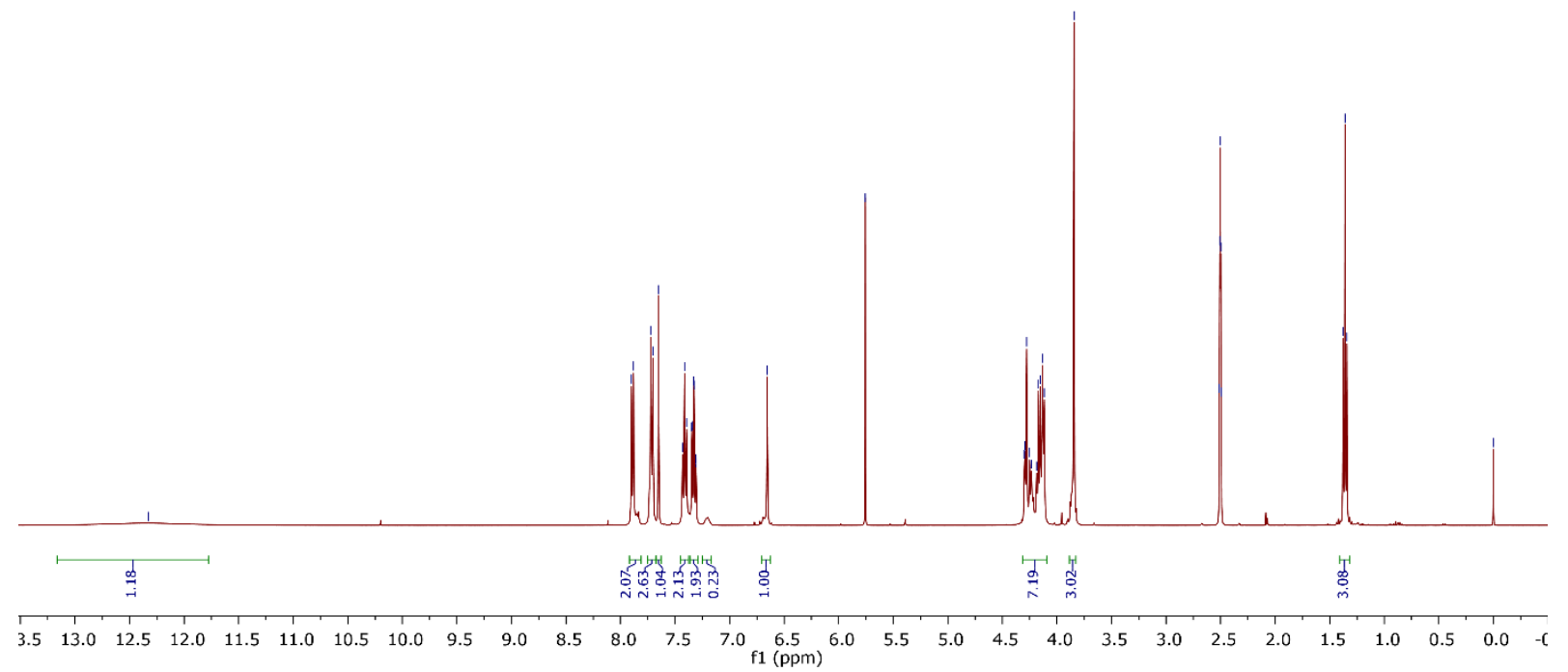

$$
\prod_{\mathrm{O}}^{\mathrm{Me}}
$$

flit.,
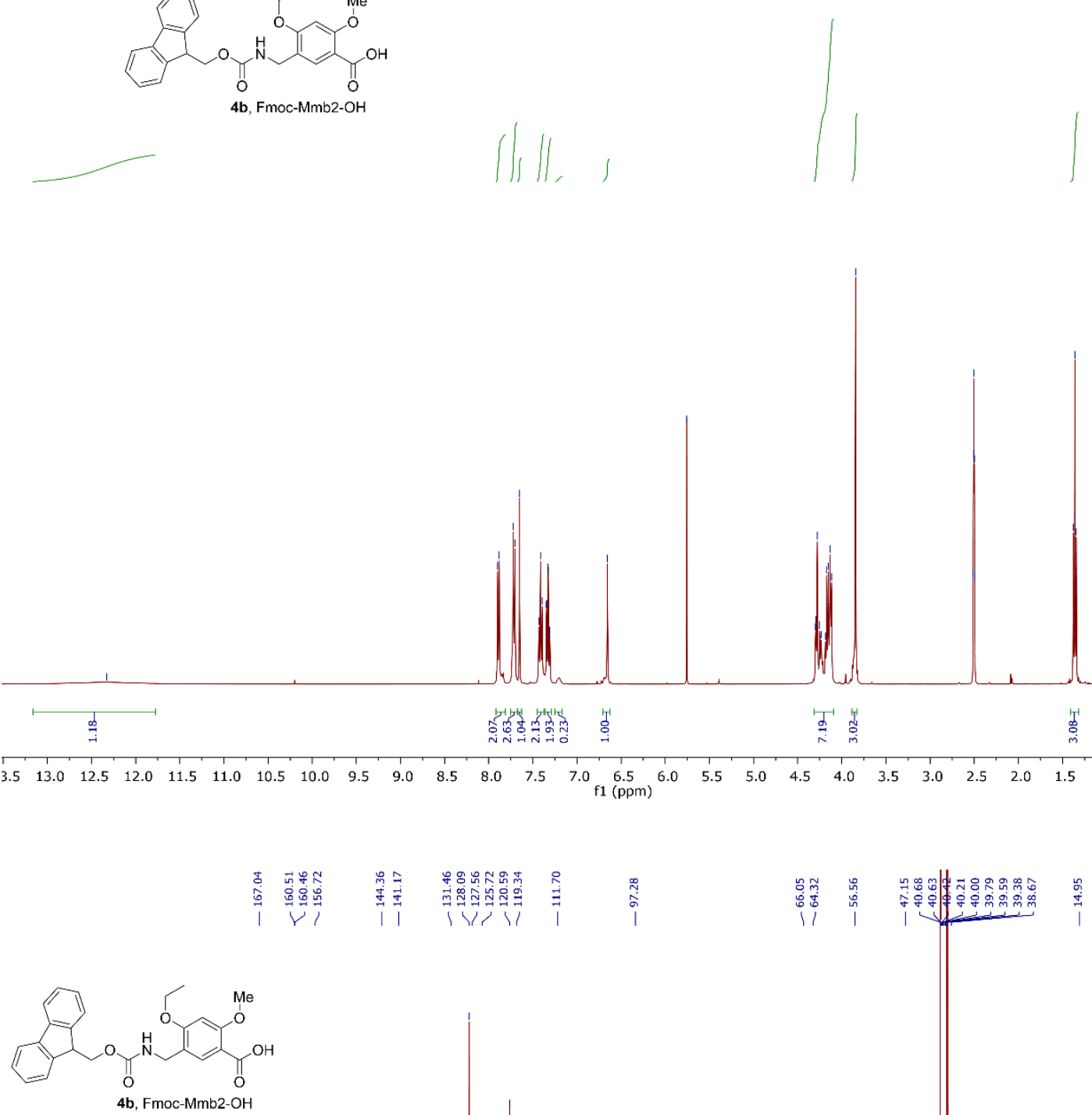

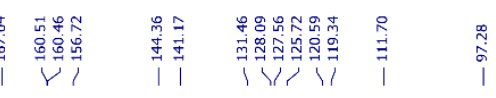
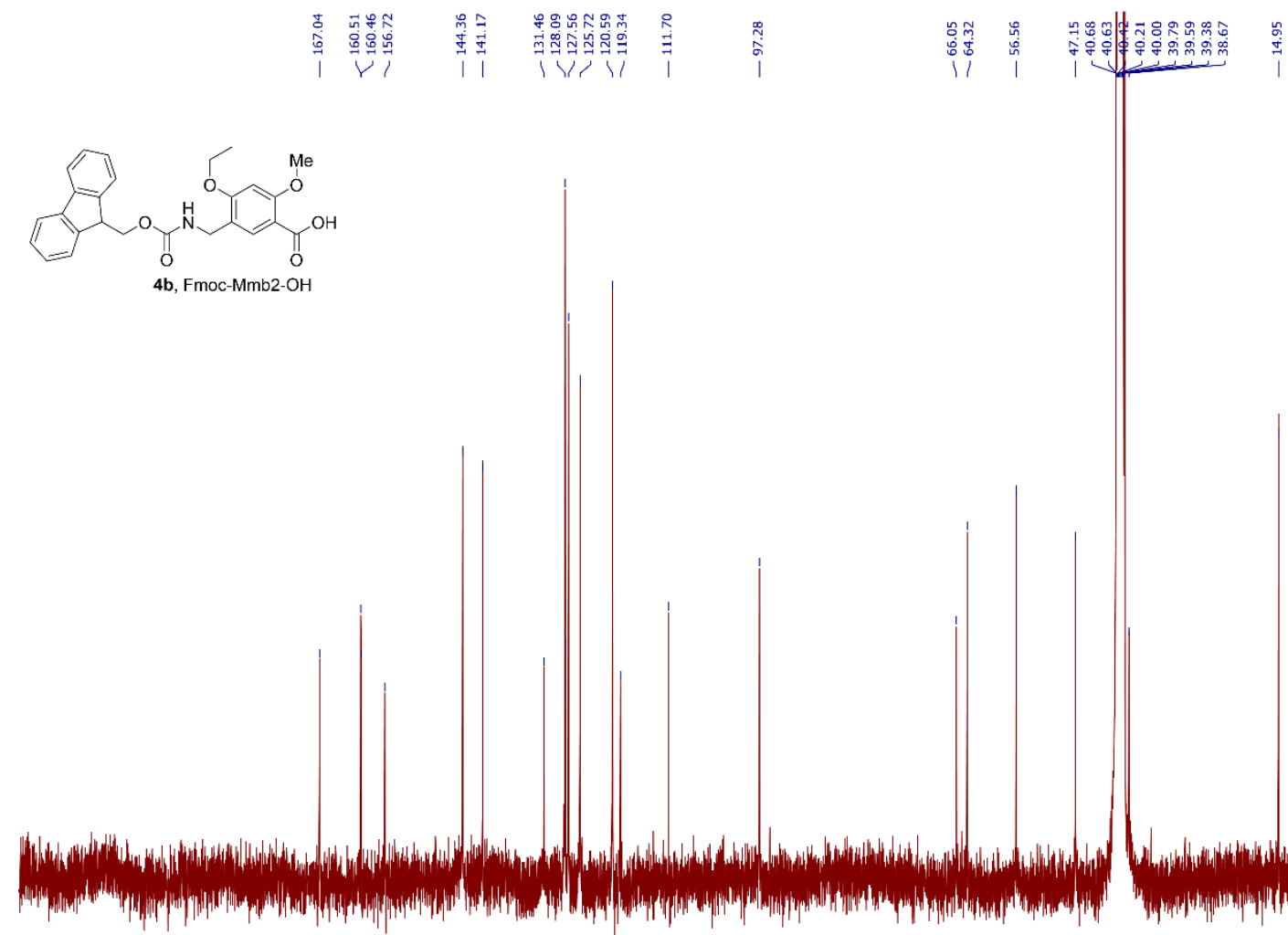

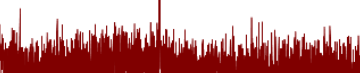

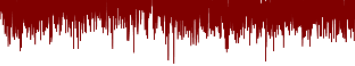

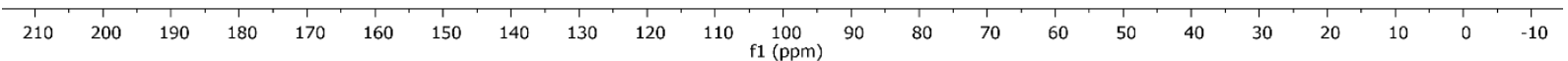



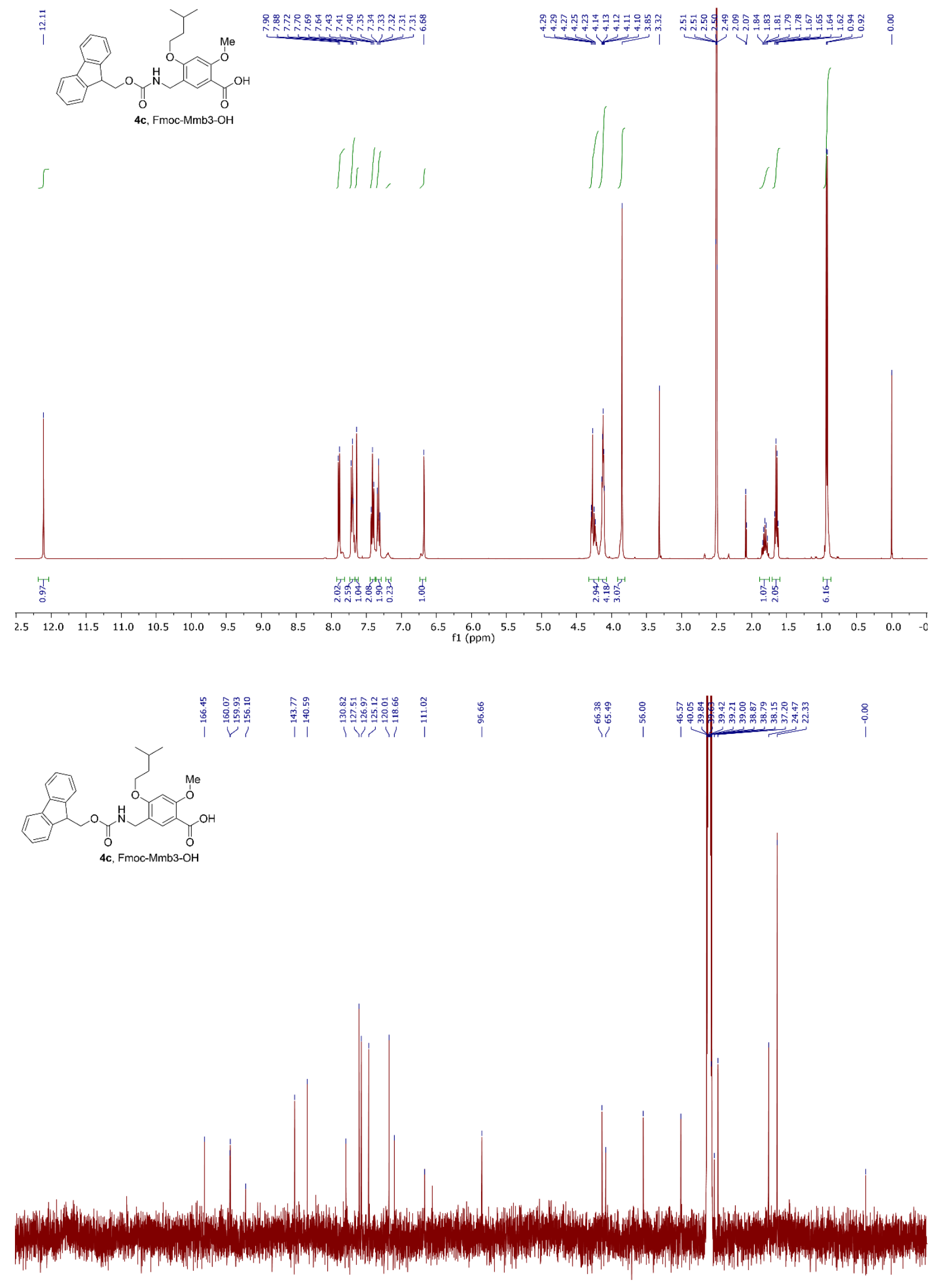

$\begin{array}{llllllllllllllllllllllllll}1 & 1 & 200 & 190 & 180 & 170 & 160 & 150 & 140 & 130 & 120 & 110 & 100 & 90 & 80 & 70 & 60 & 50 & 40 & 30 & 20 & 10 & 0 & -10\end{array}$ 

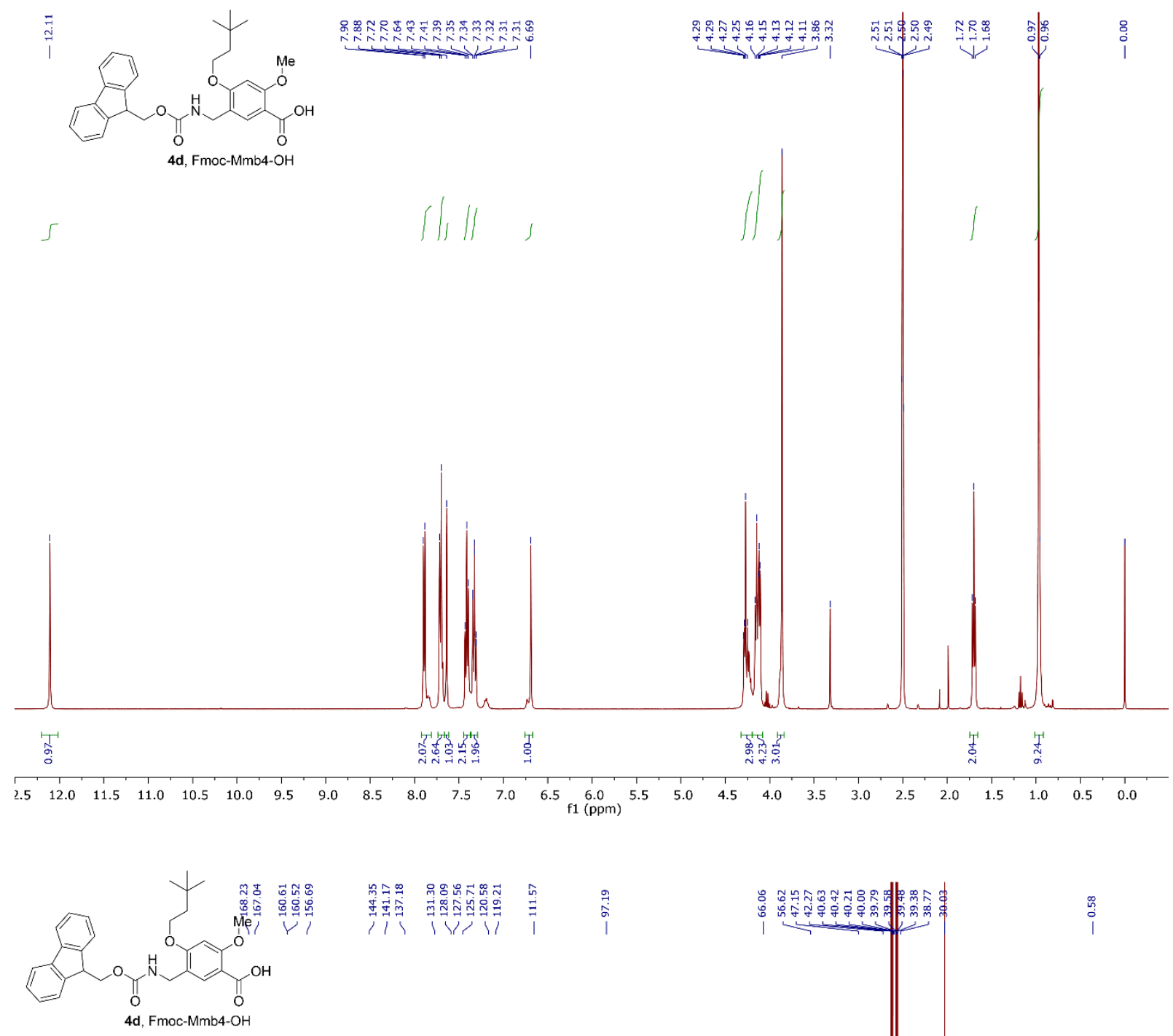

i
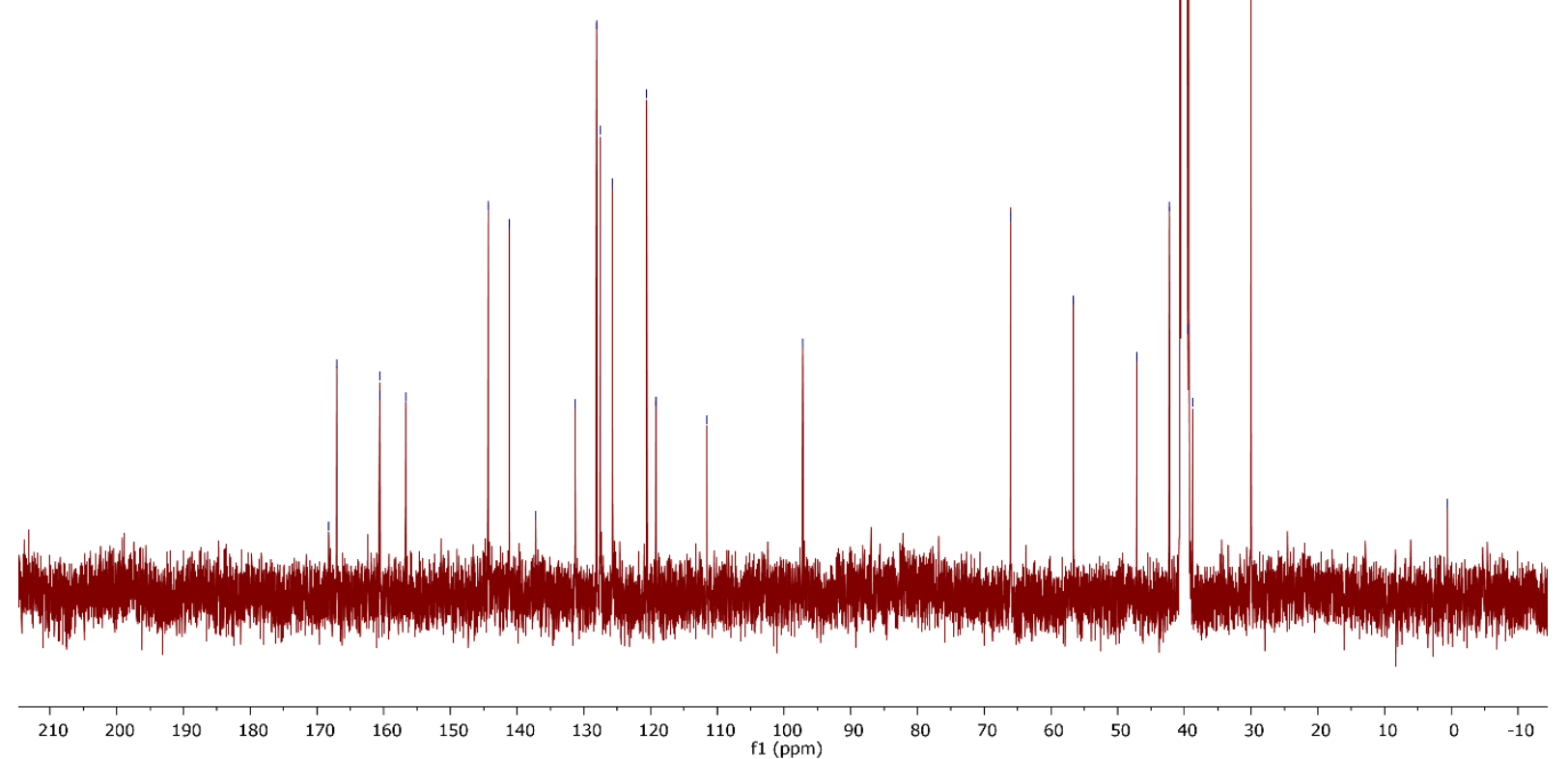

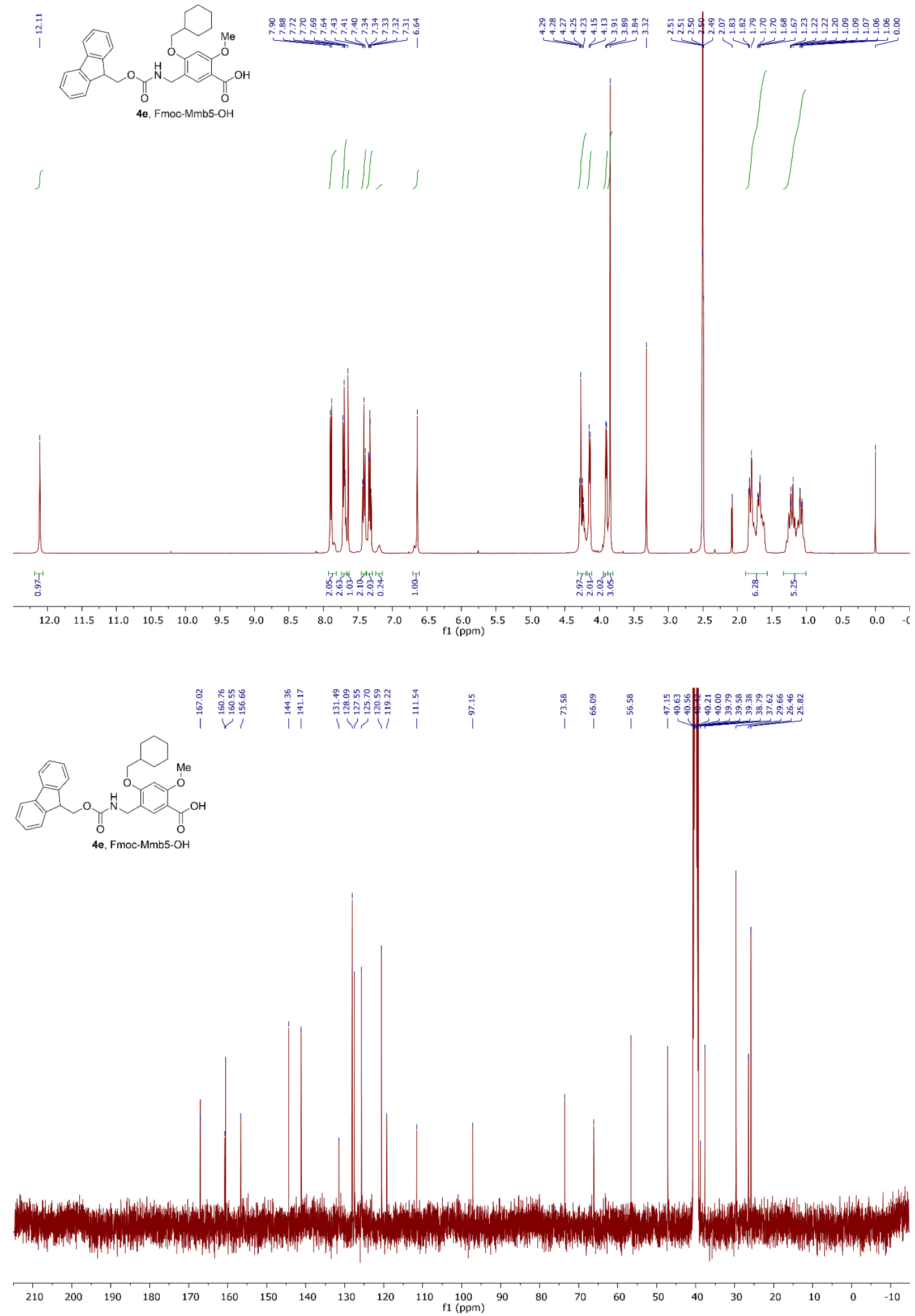

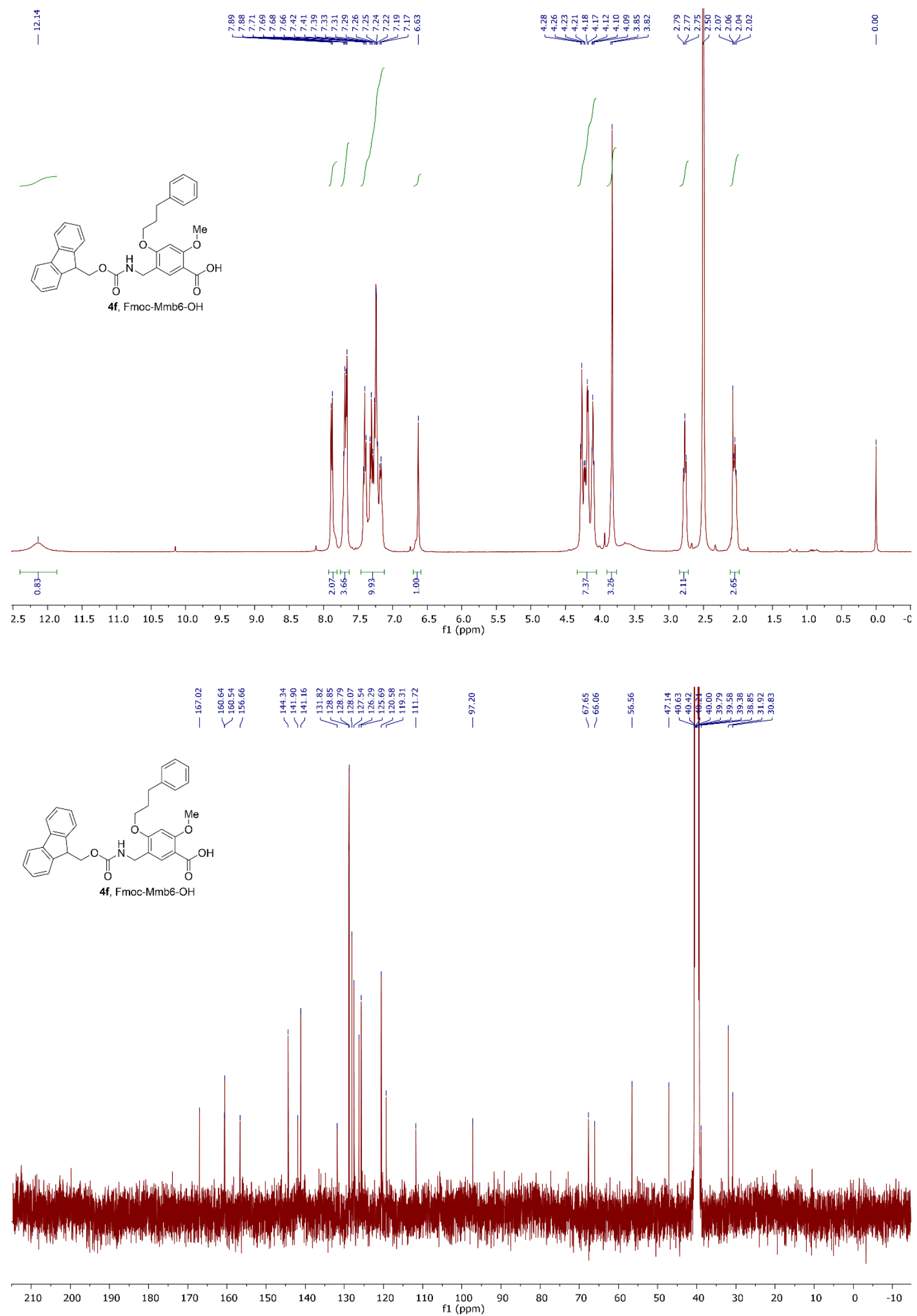


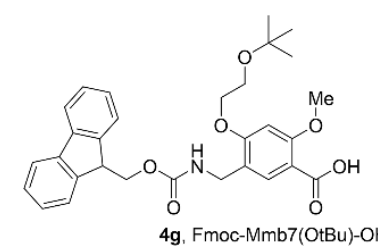

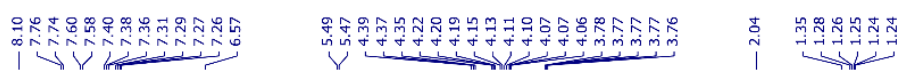
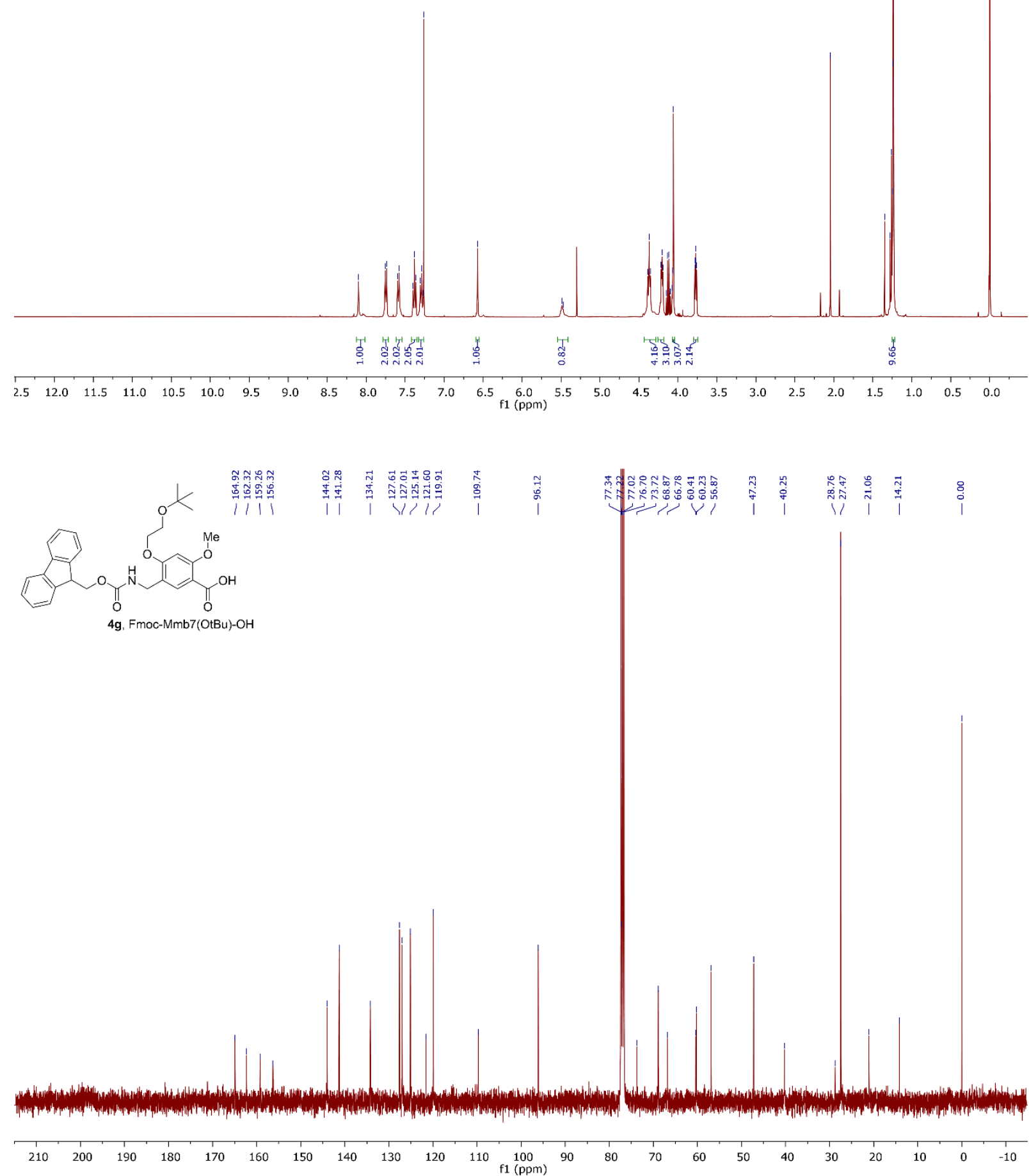

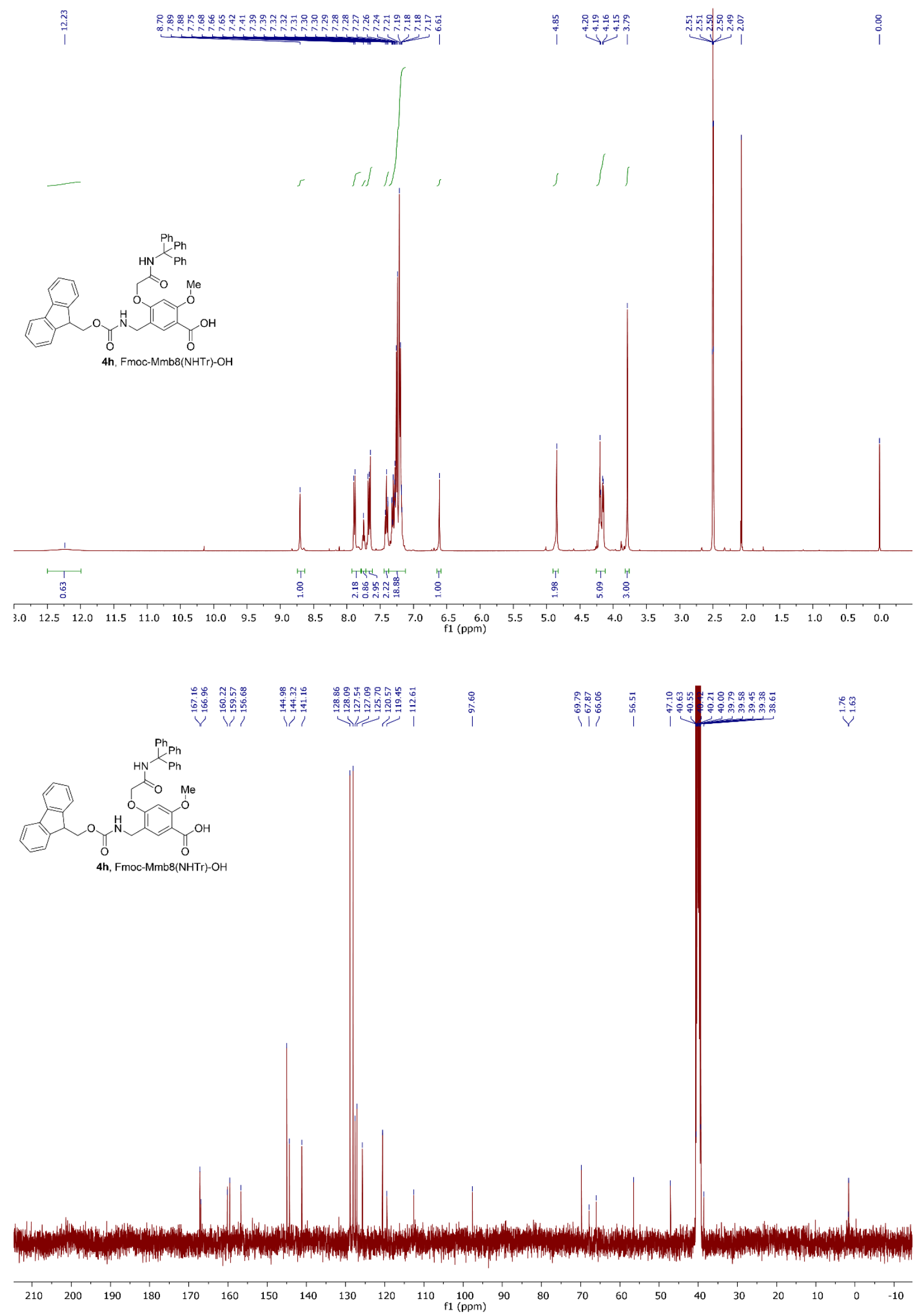

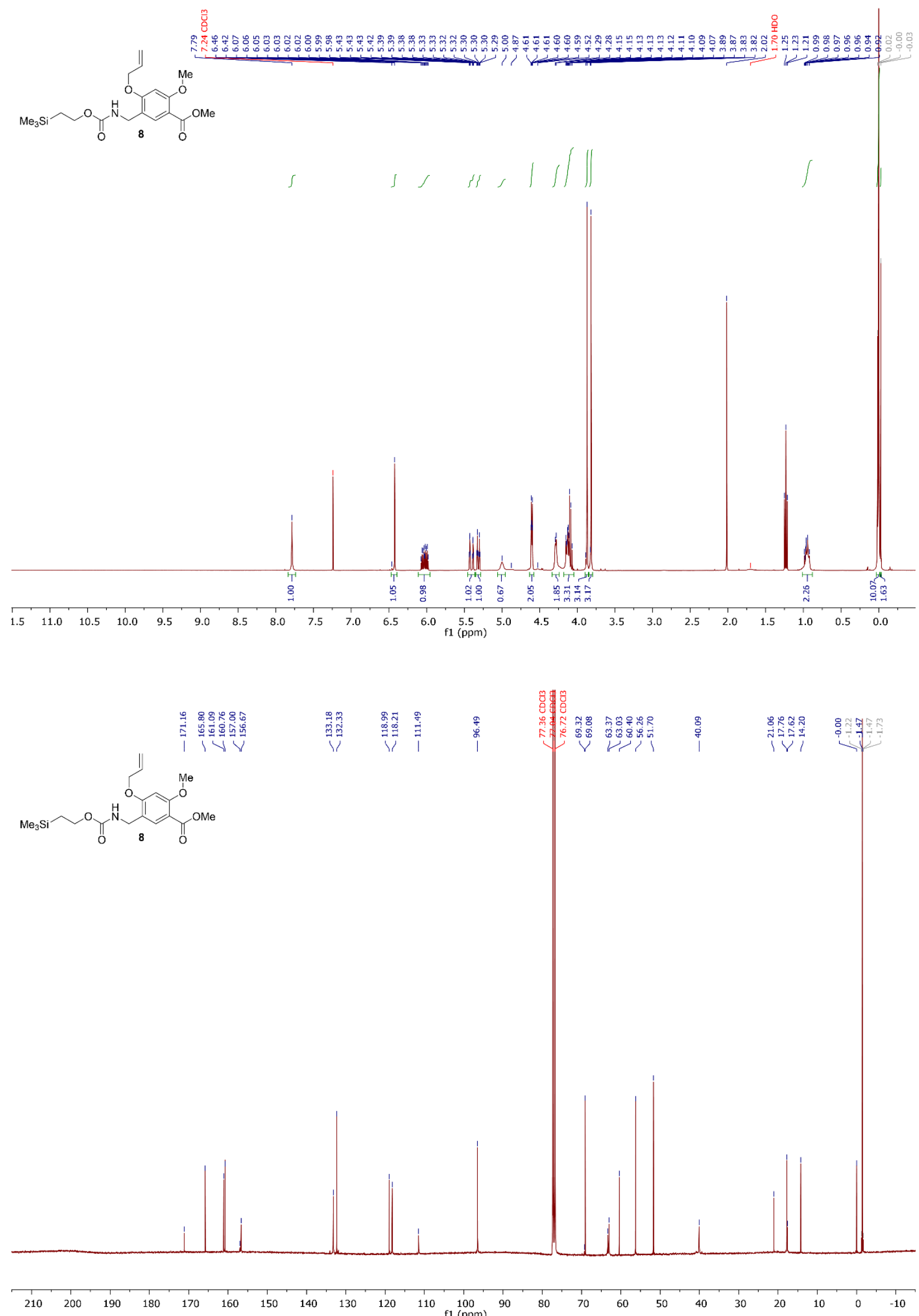

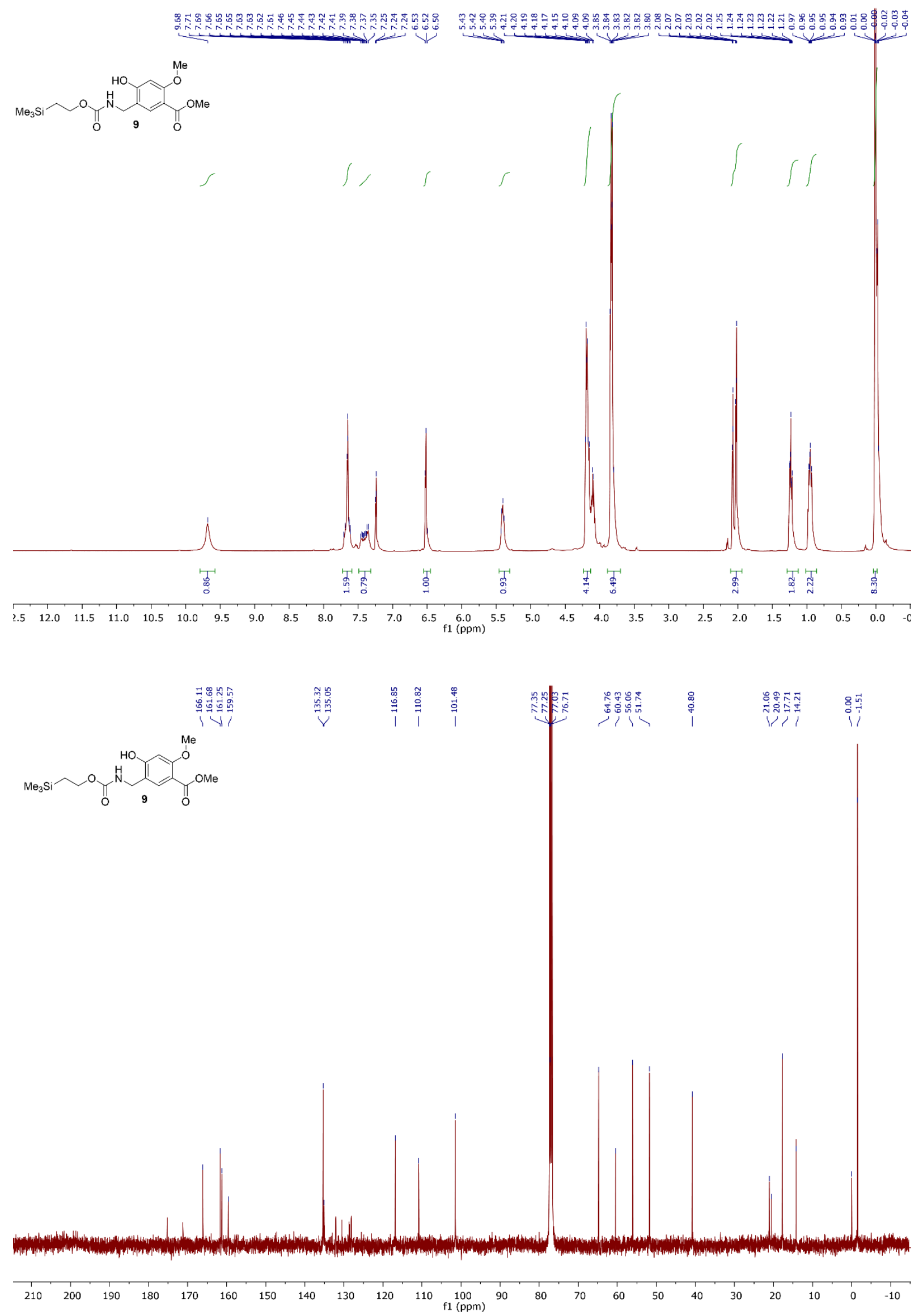

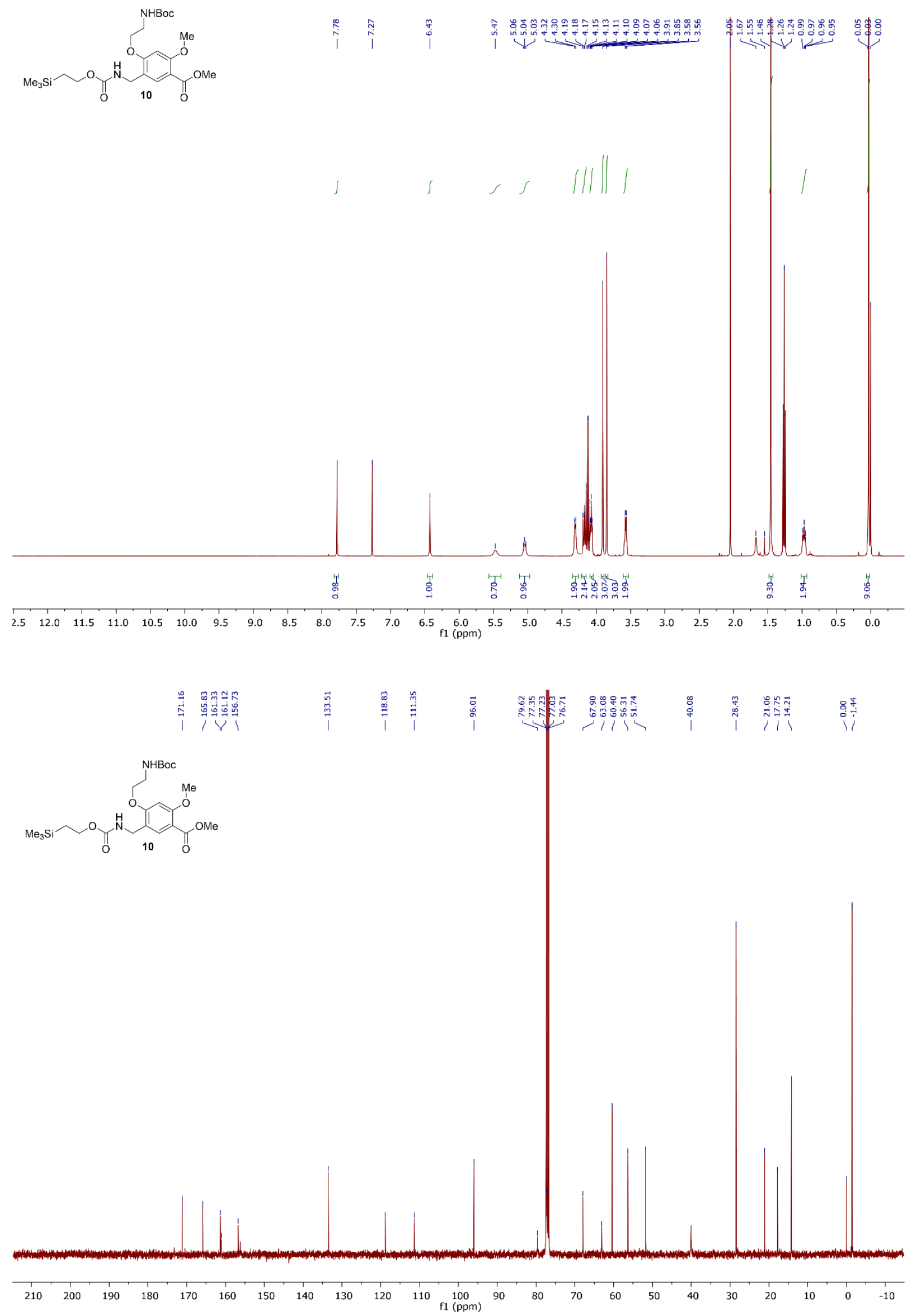


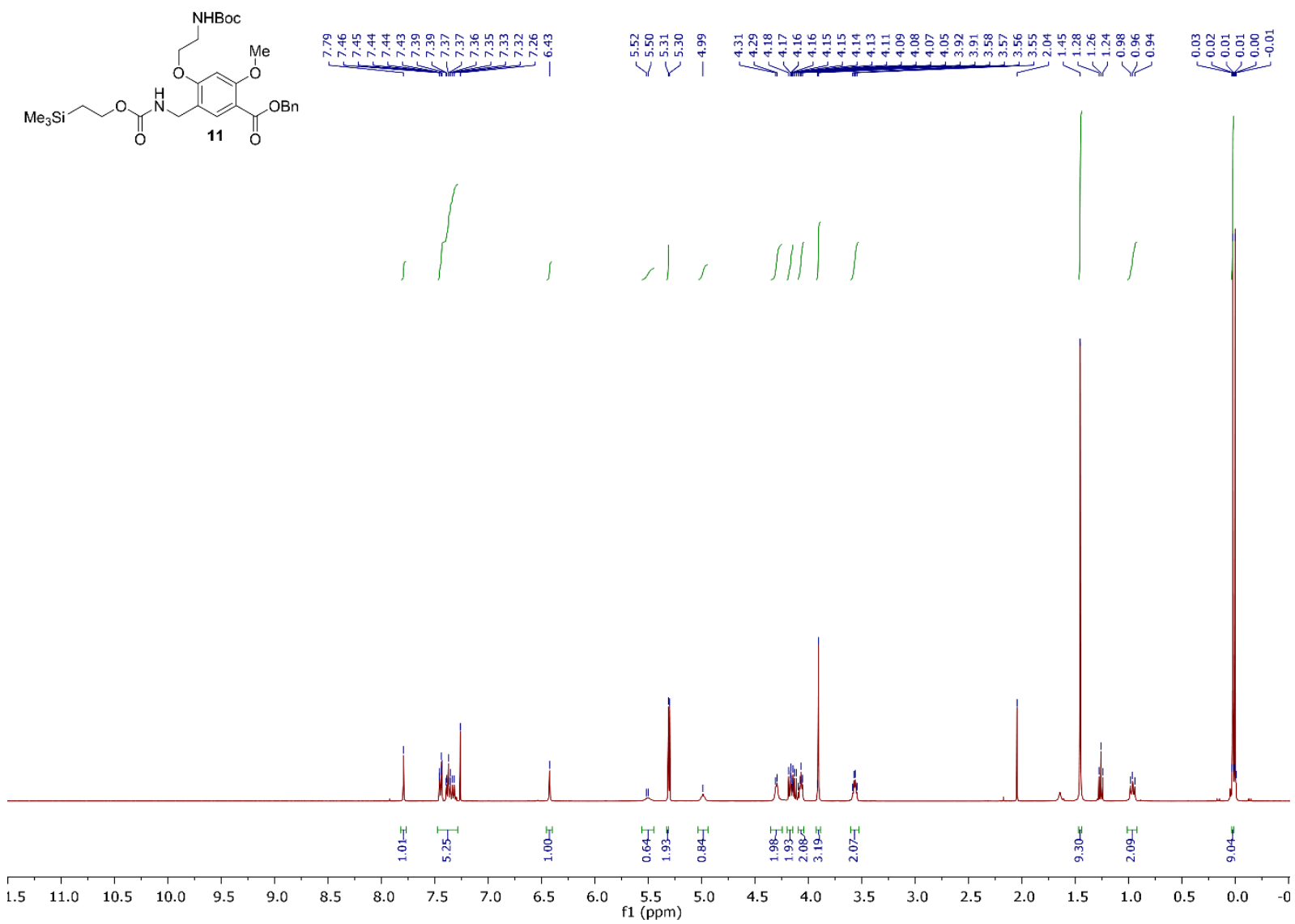

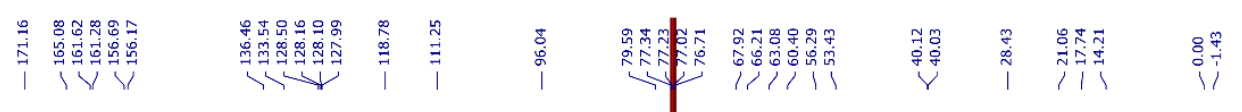
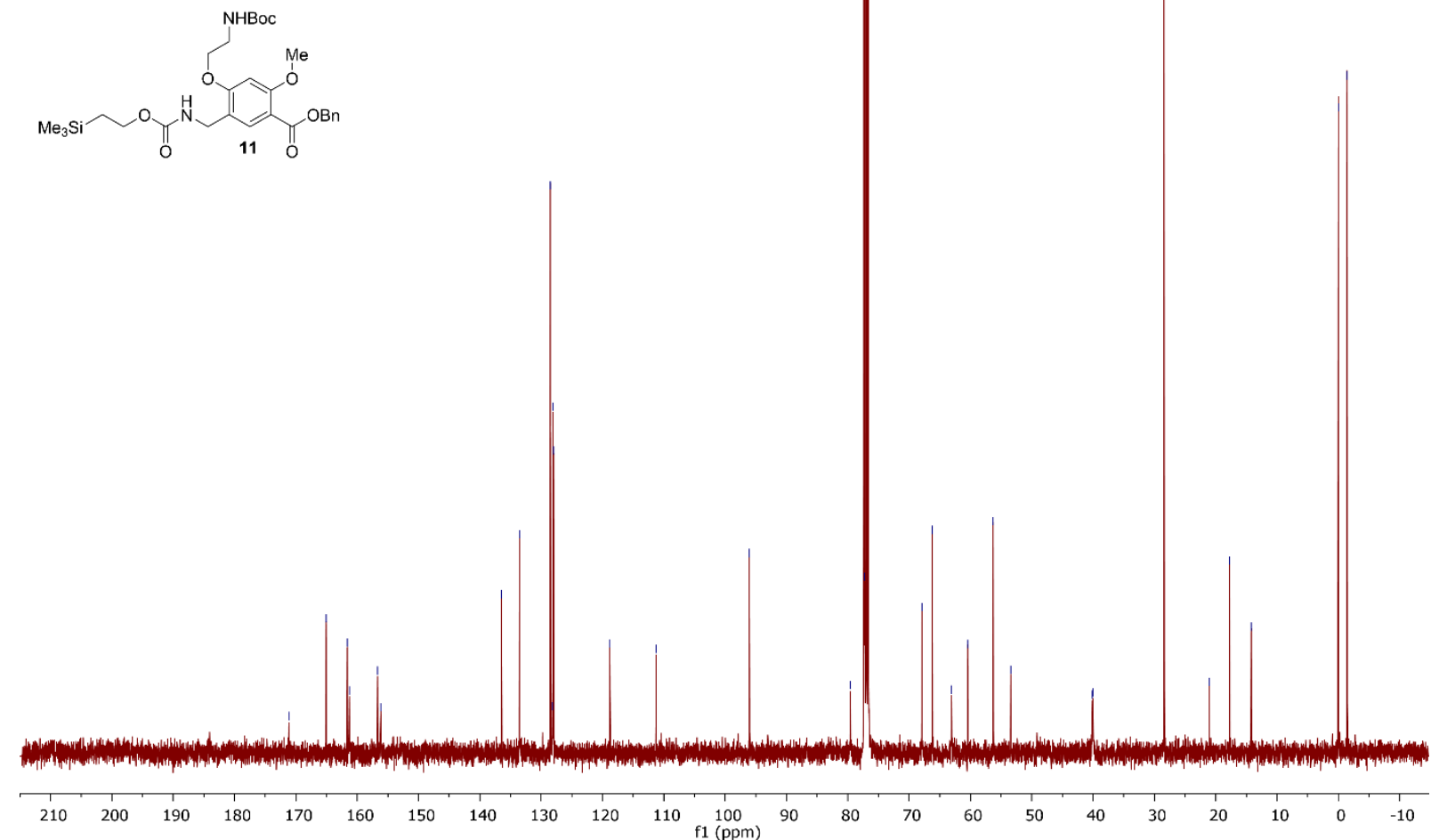

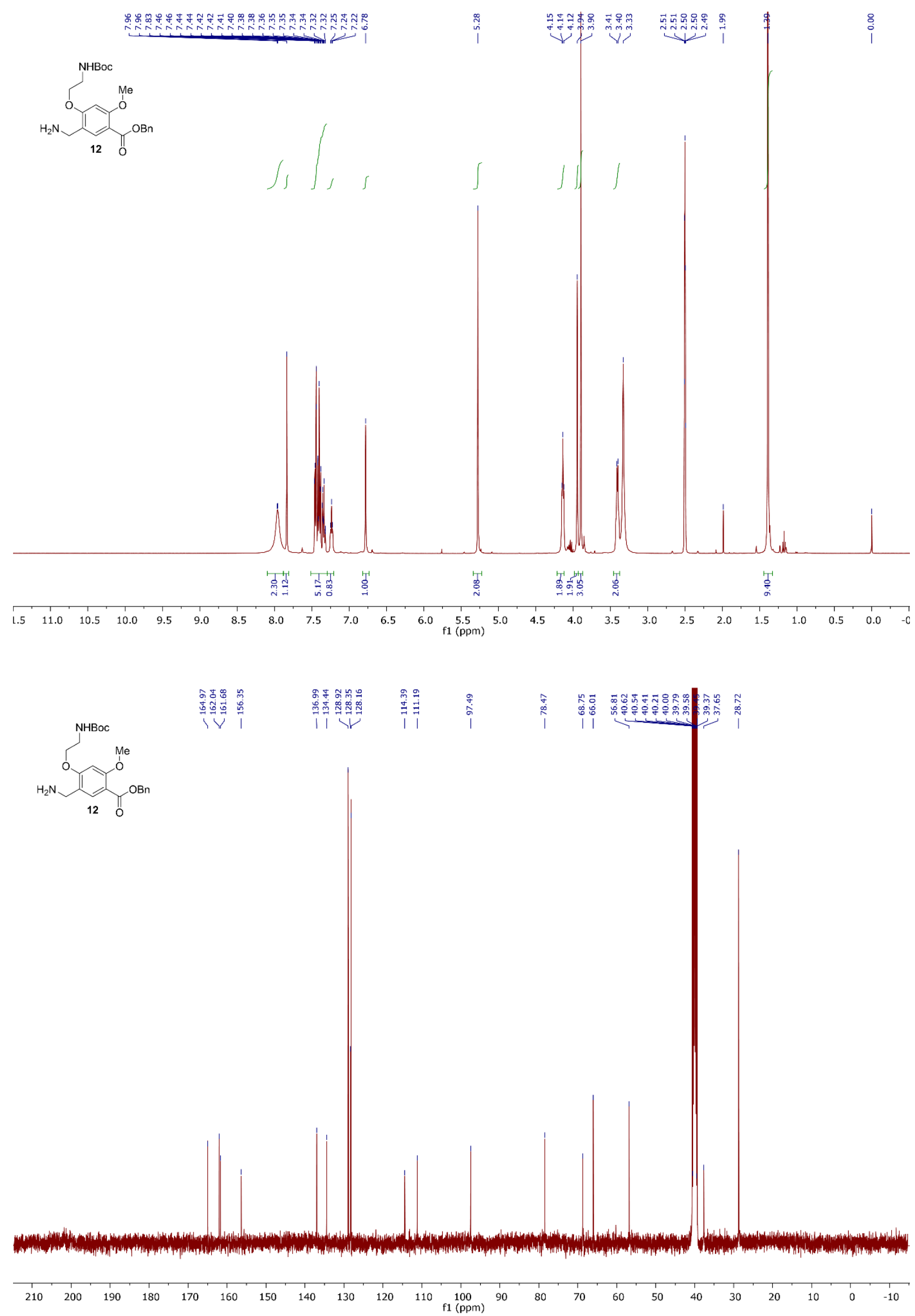

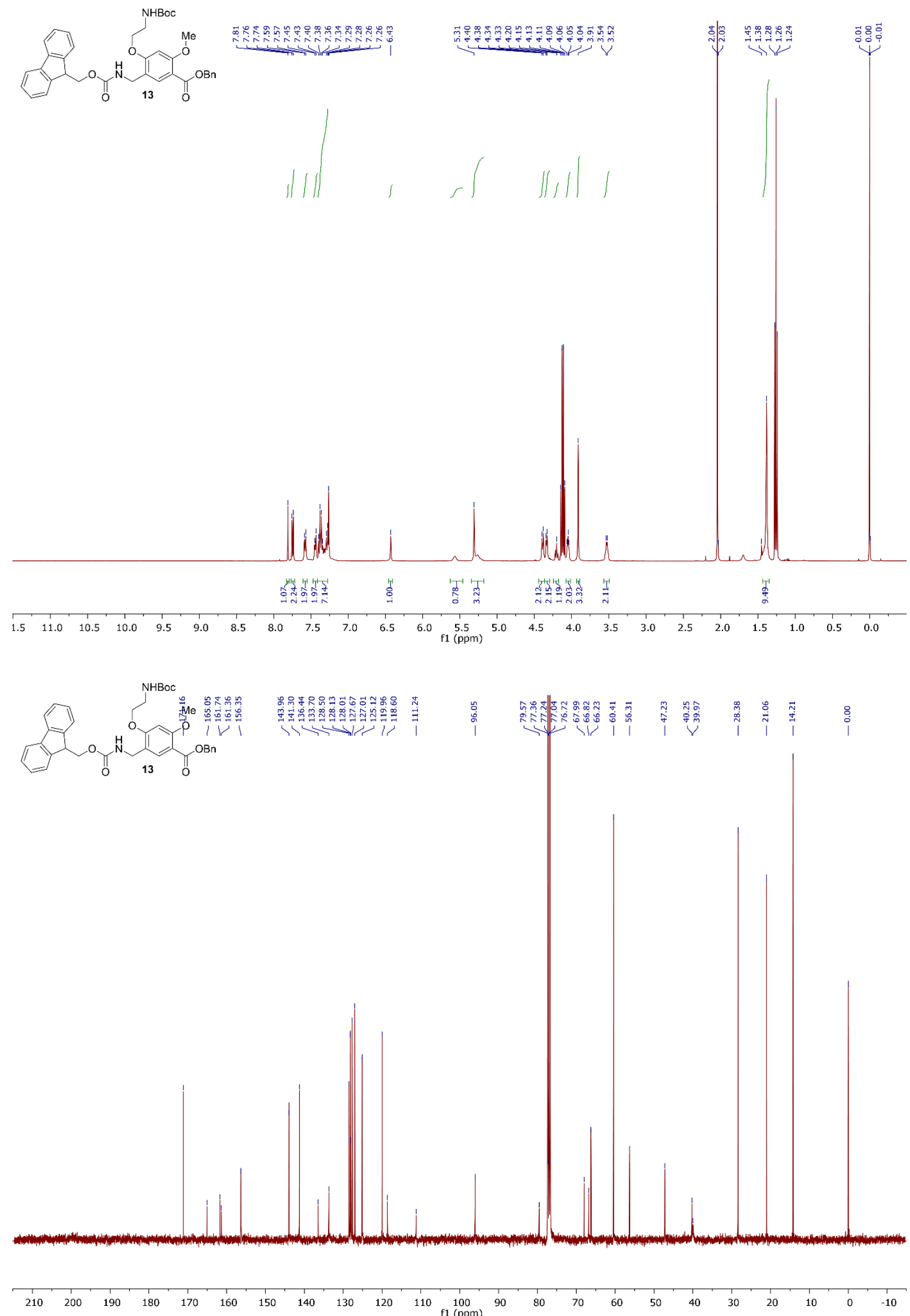


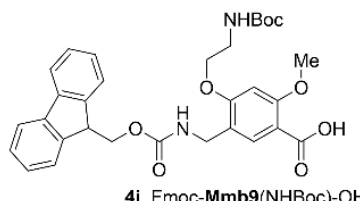

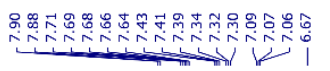

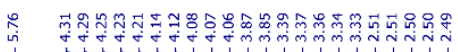

i. Fmoc-Mmb9(NHBoc)-OH

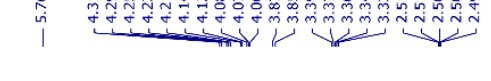$$
\text { s }
$$
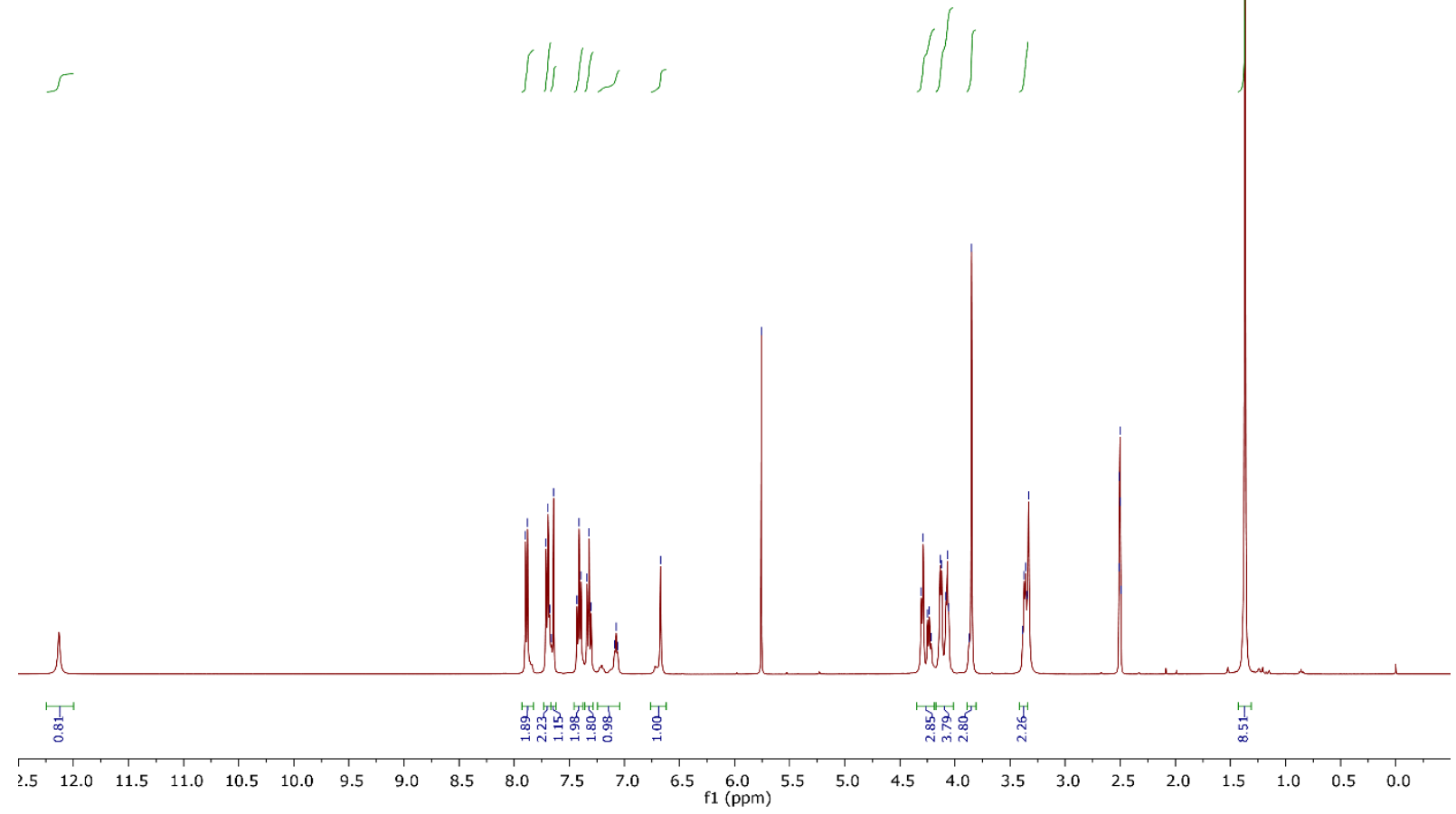

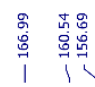

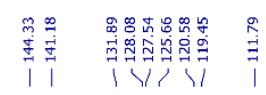

å

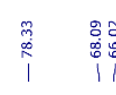

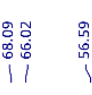

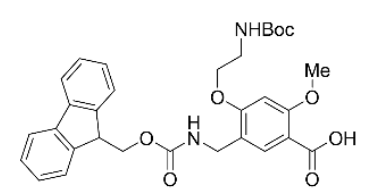

4i, Fmoc-Mmb9(NHBoc)-OH

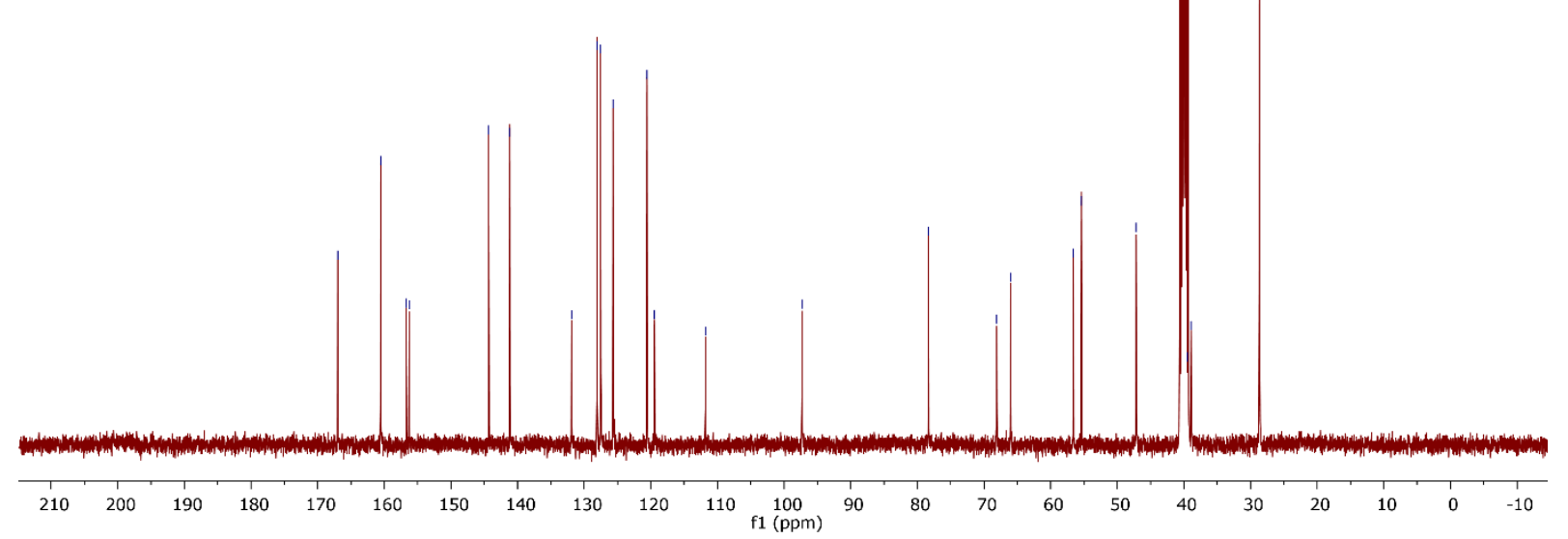




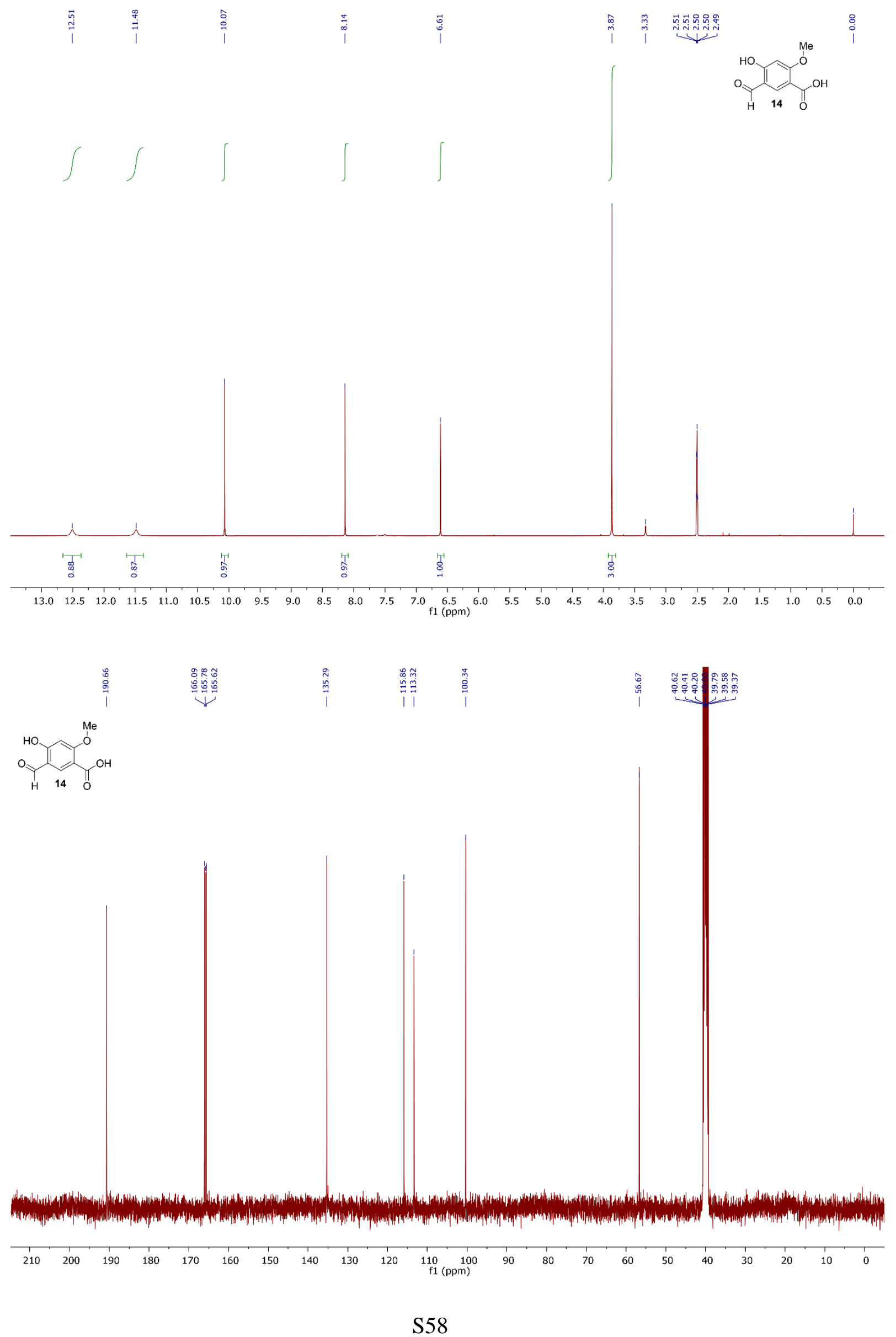




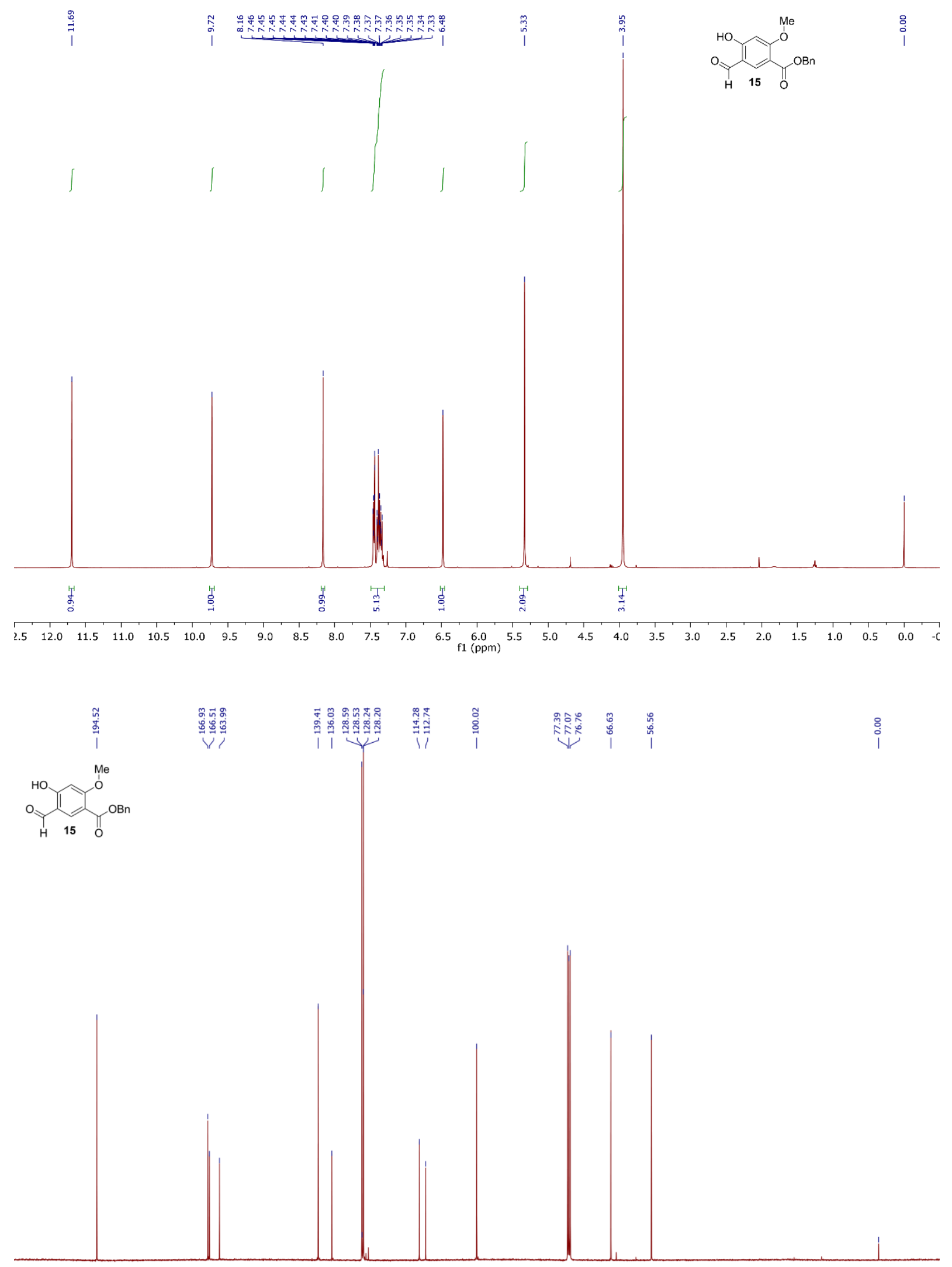

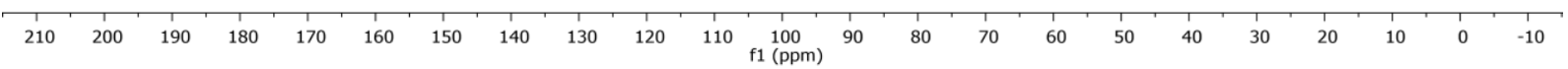



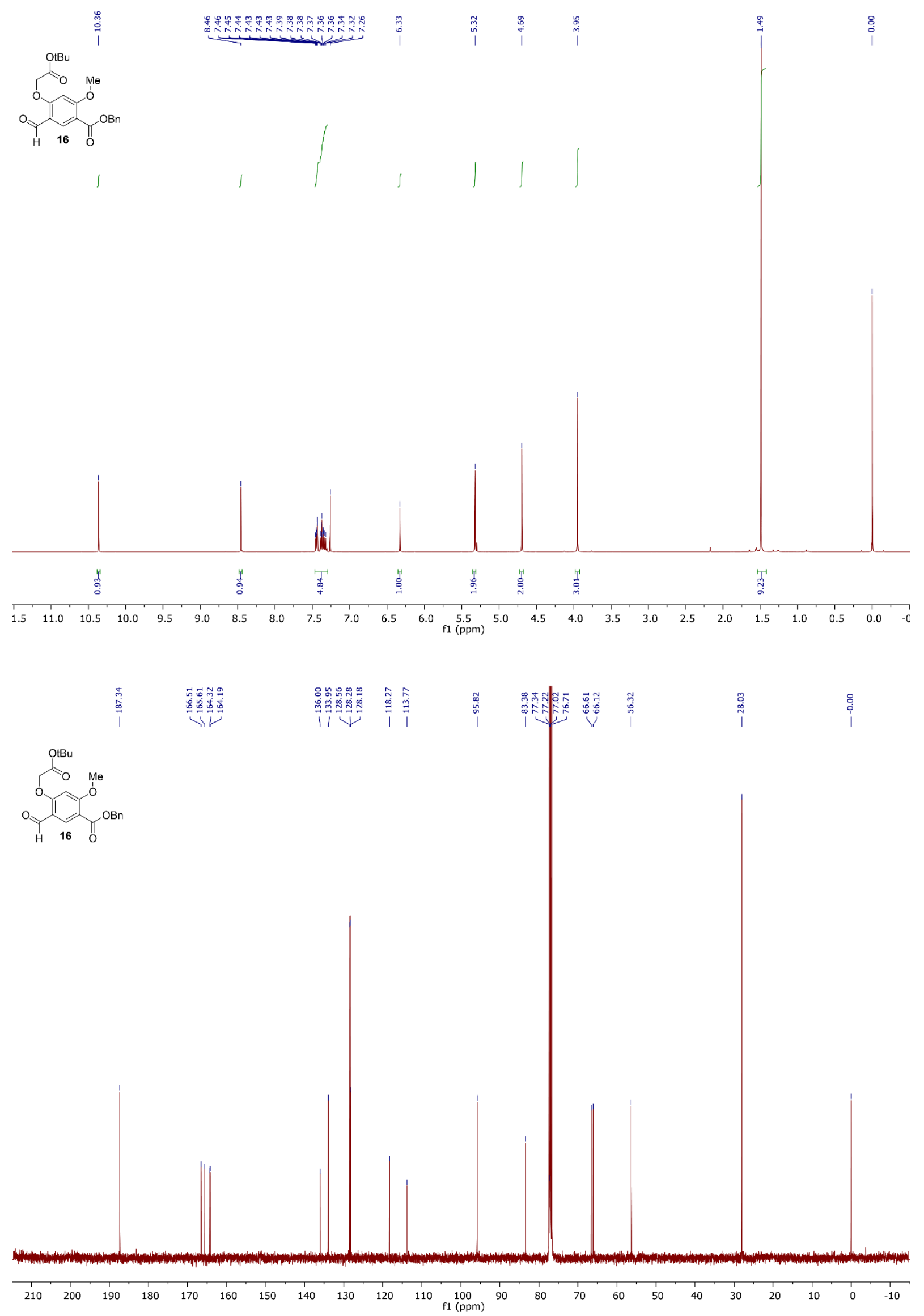

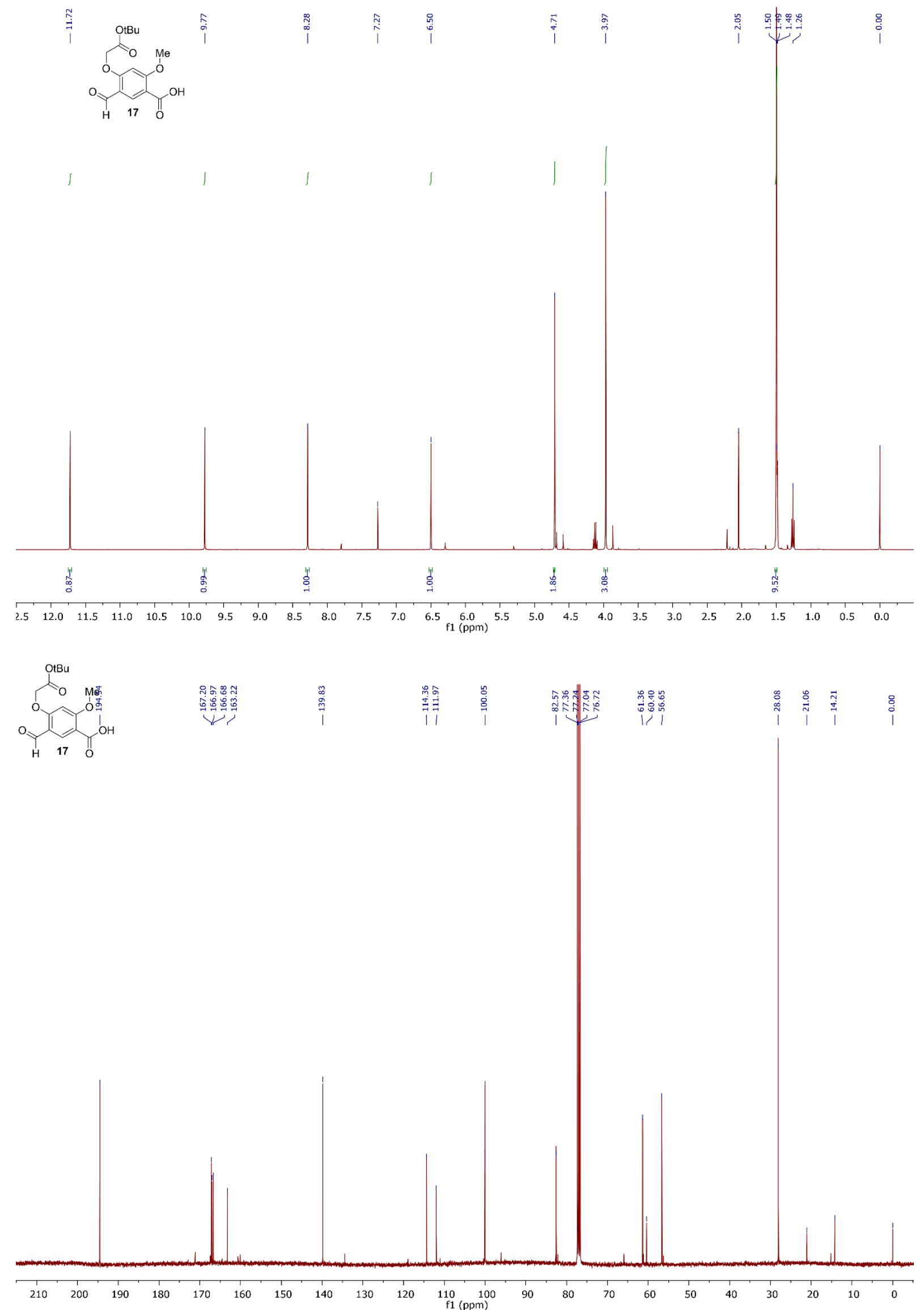

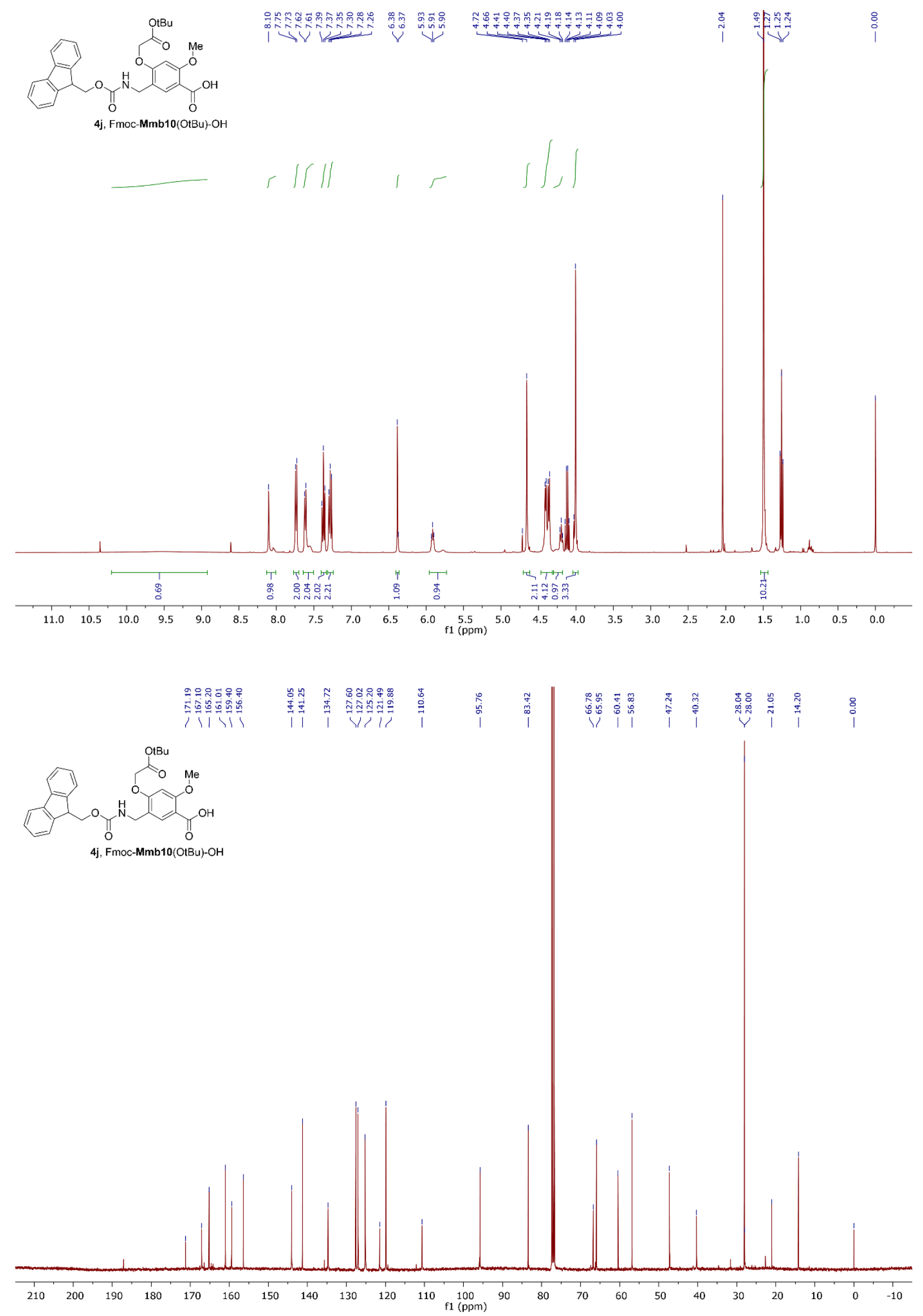


\subsection{Macrocycle 1D and 2D NOESY NMR Spectra}

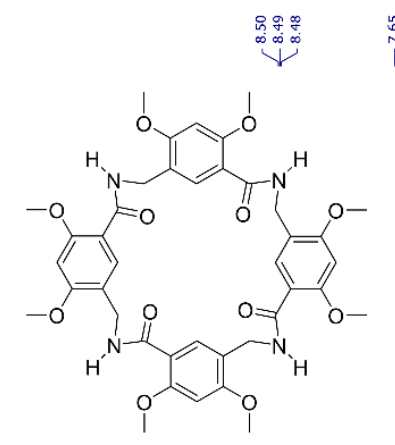

cyclo(Mmb1) ${ }_{4}$
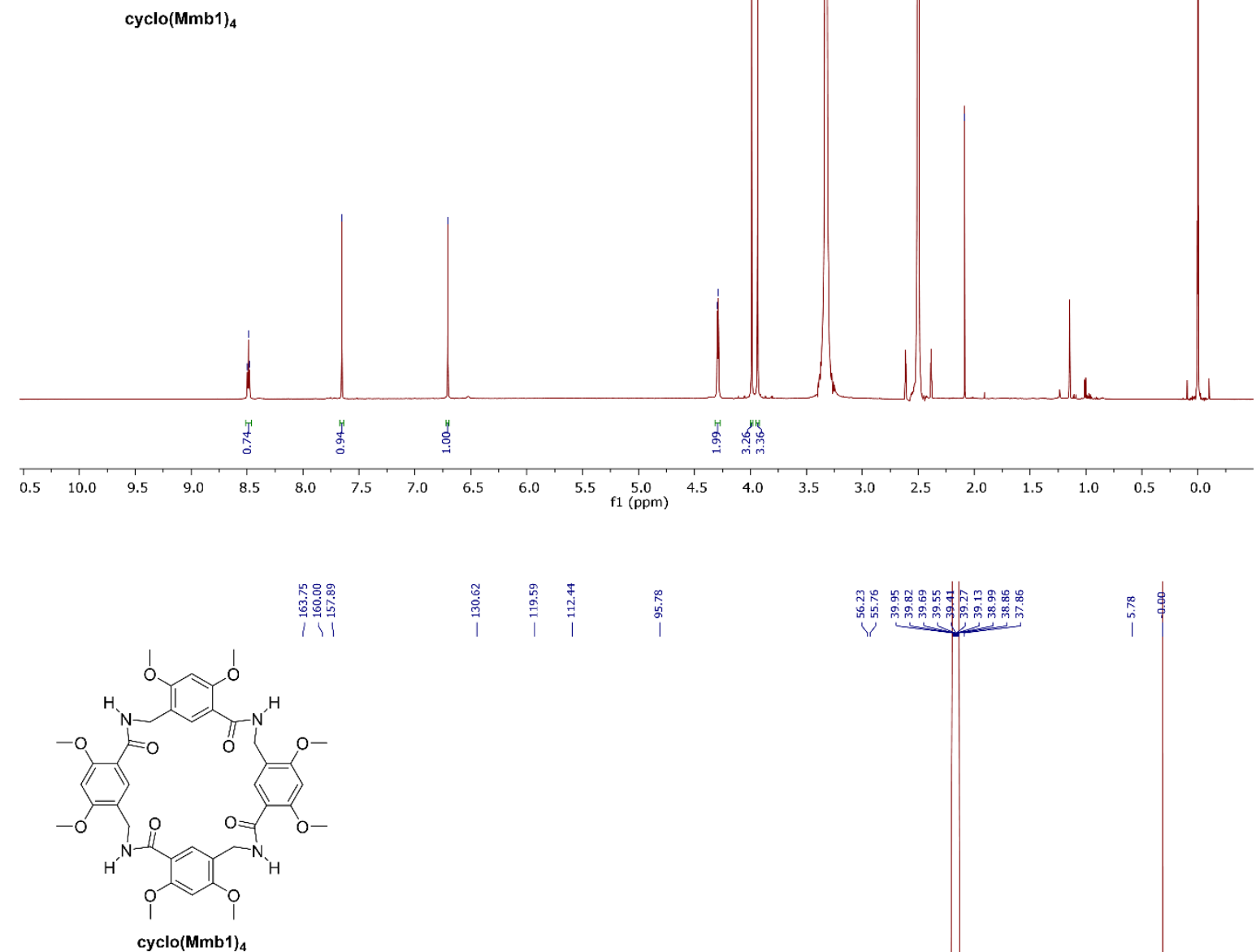

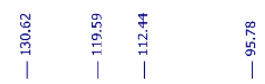
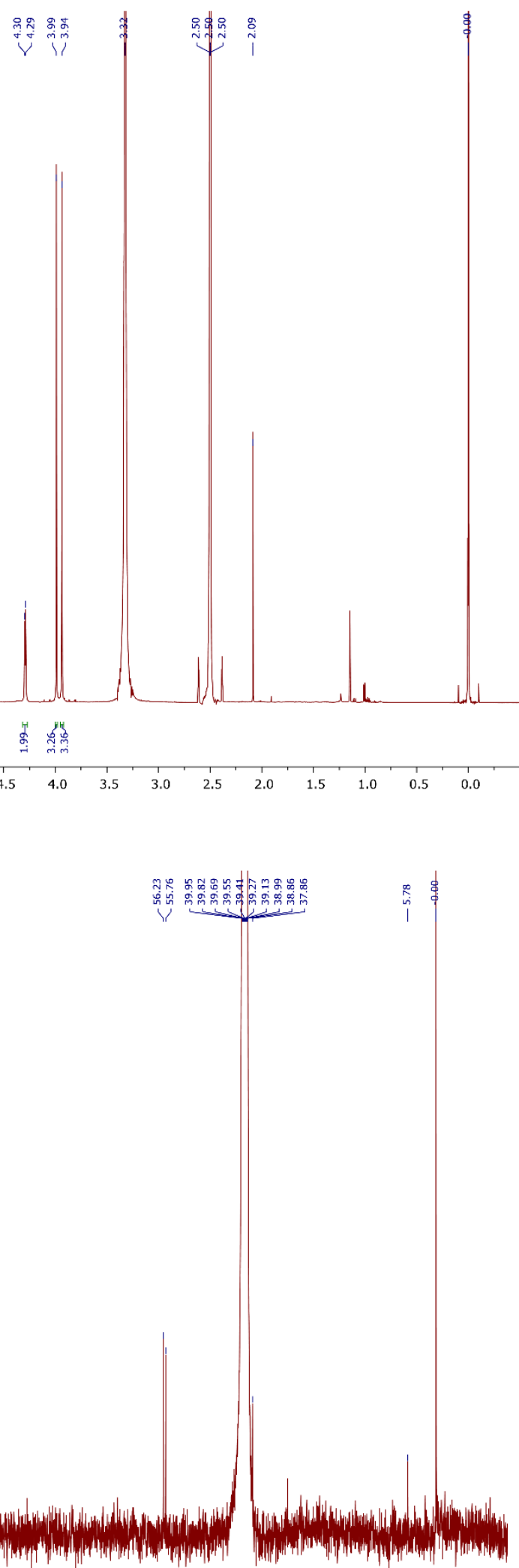

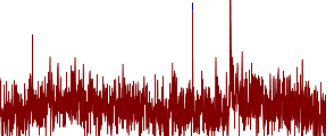

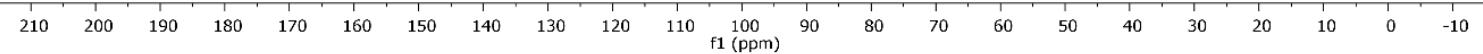



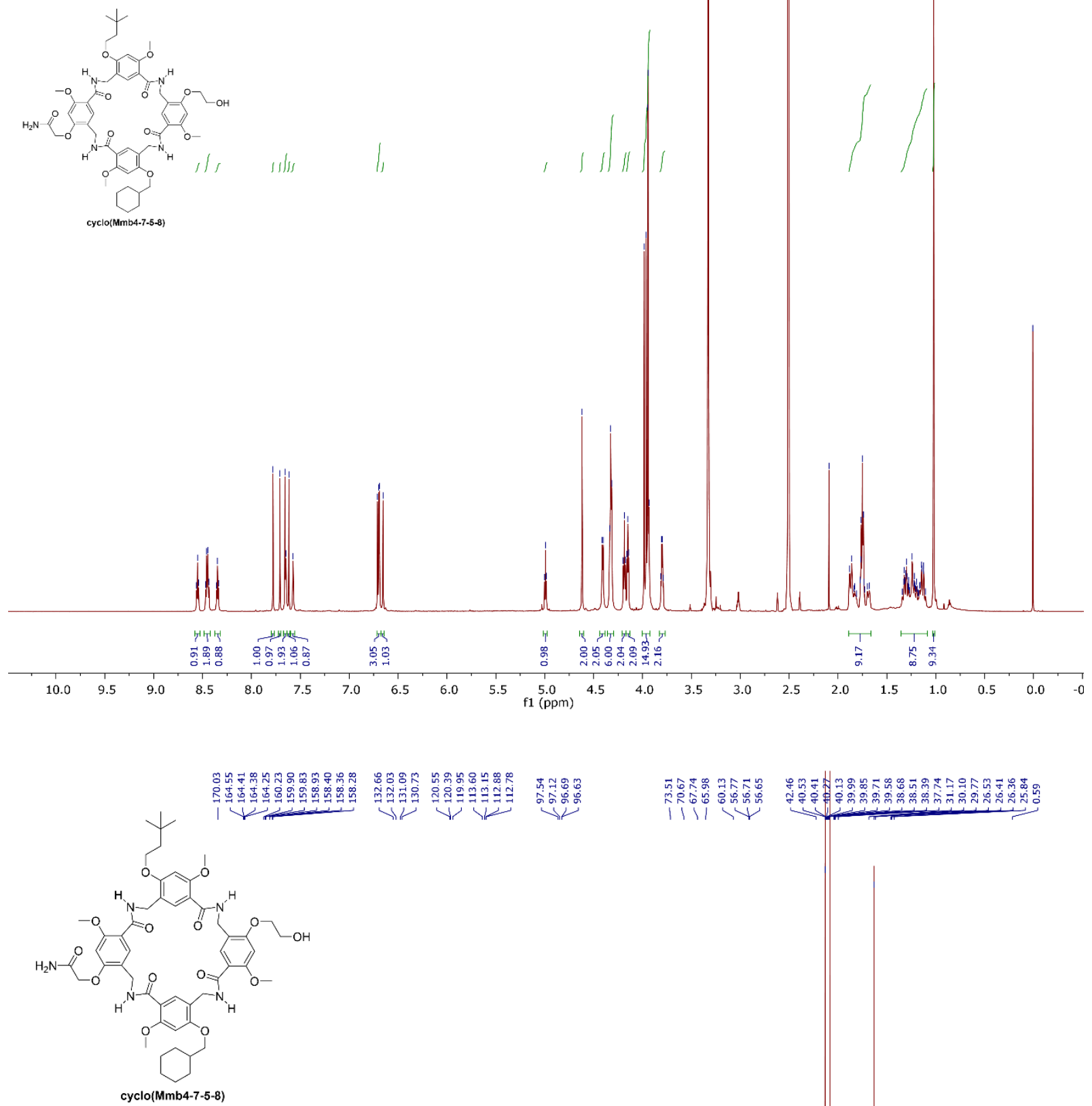

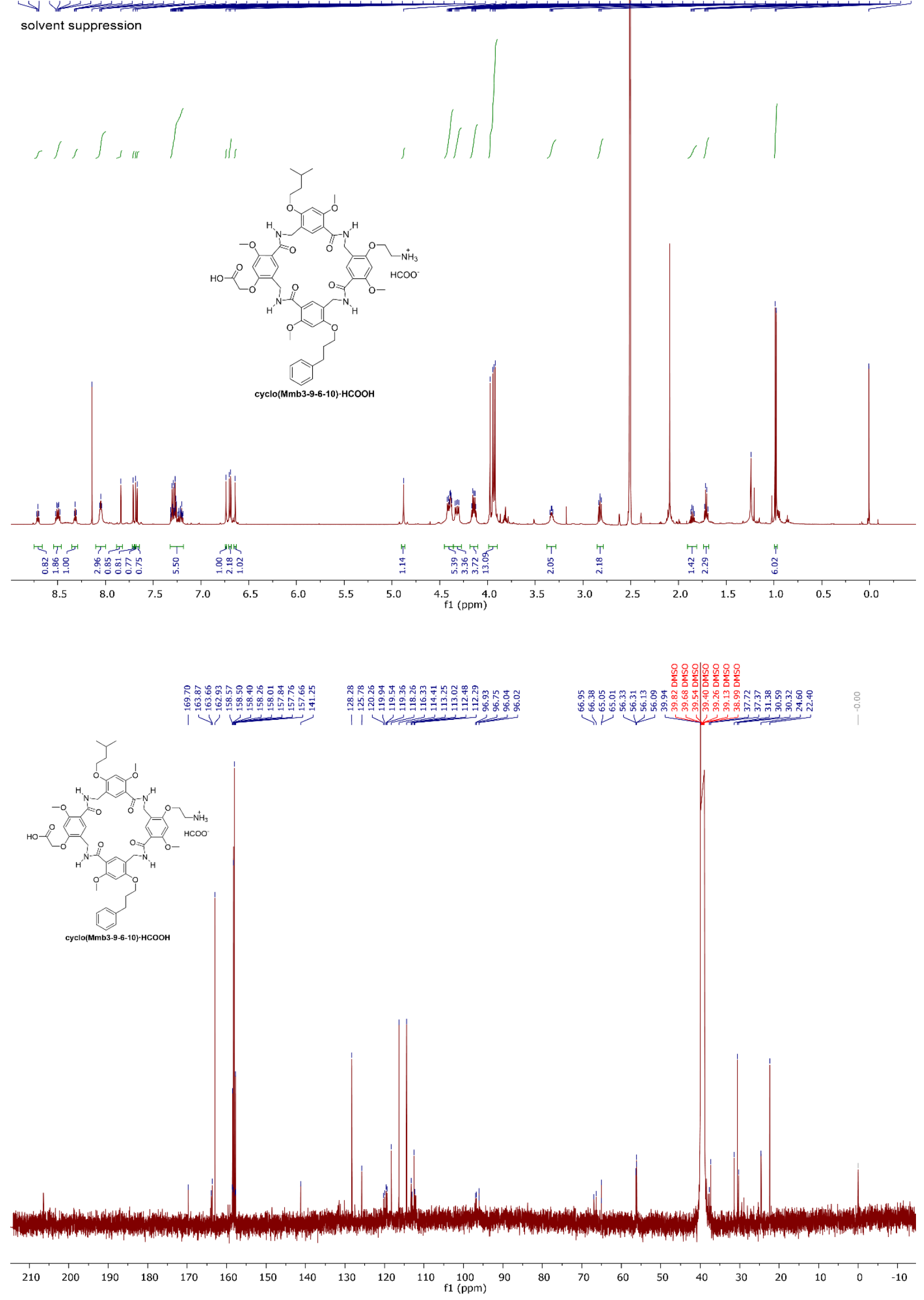

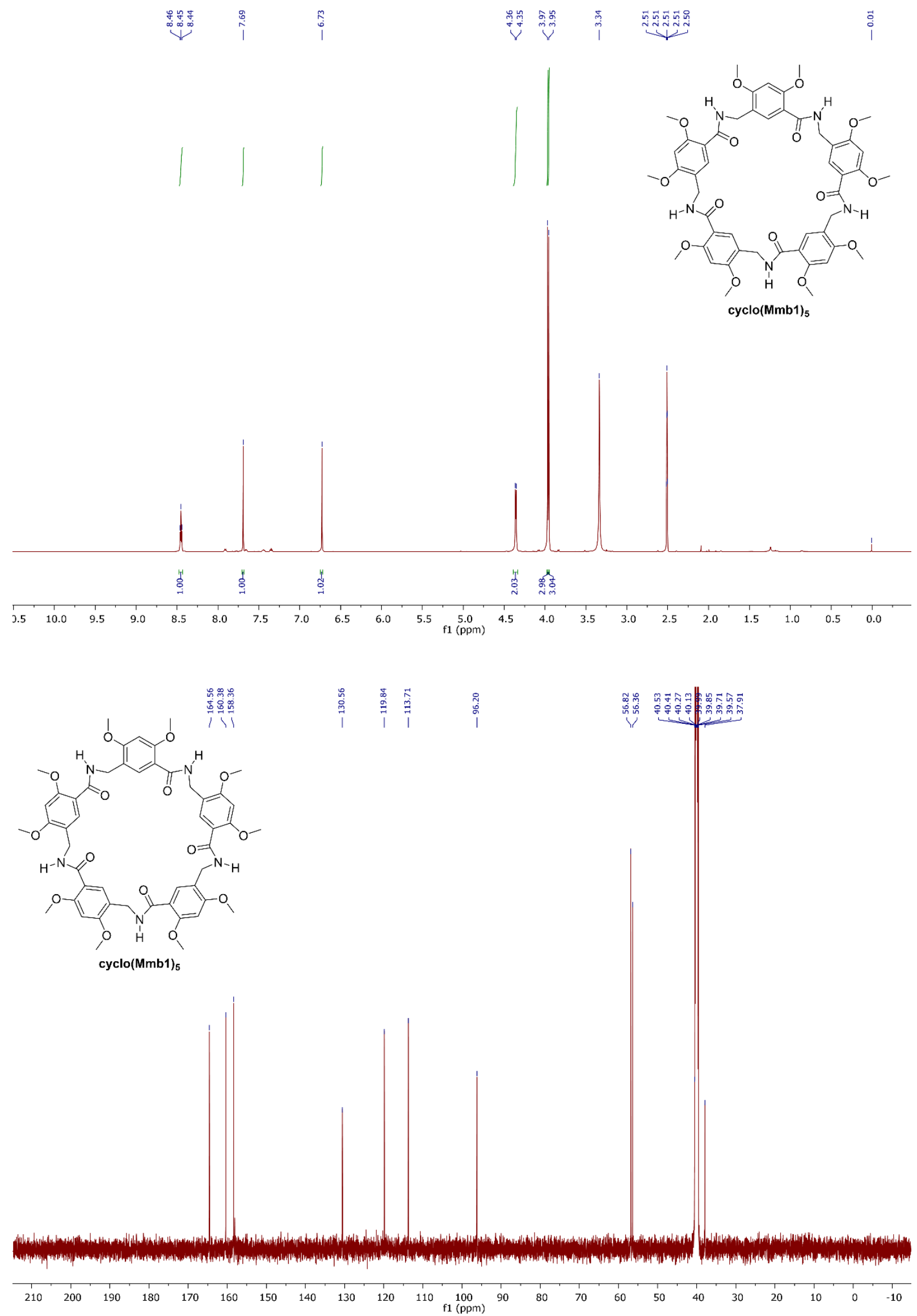

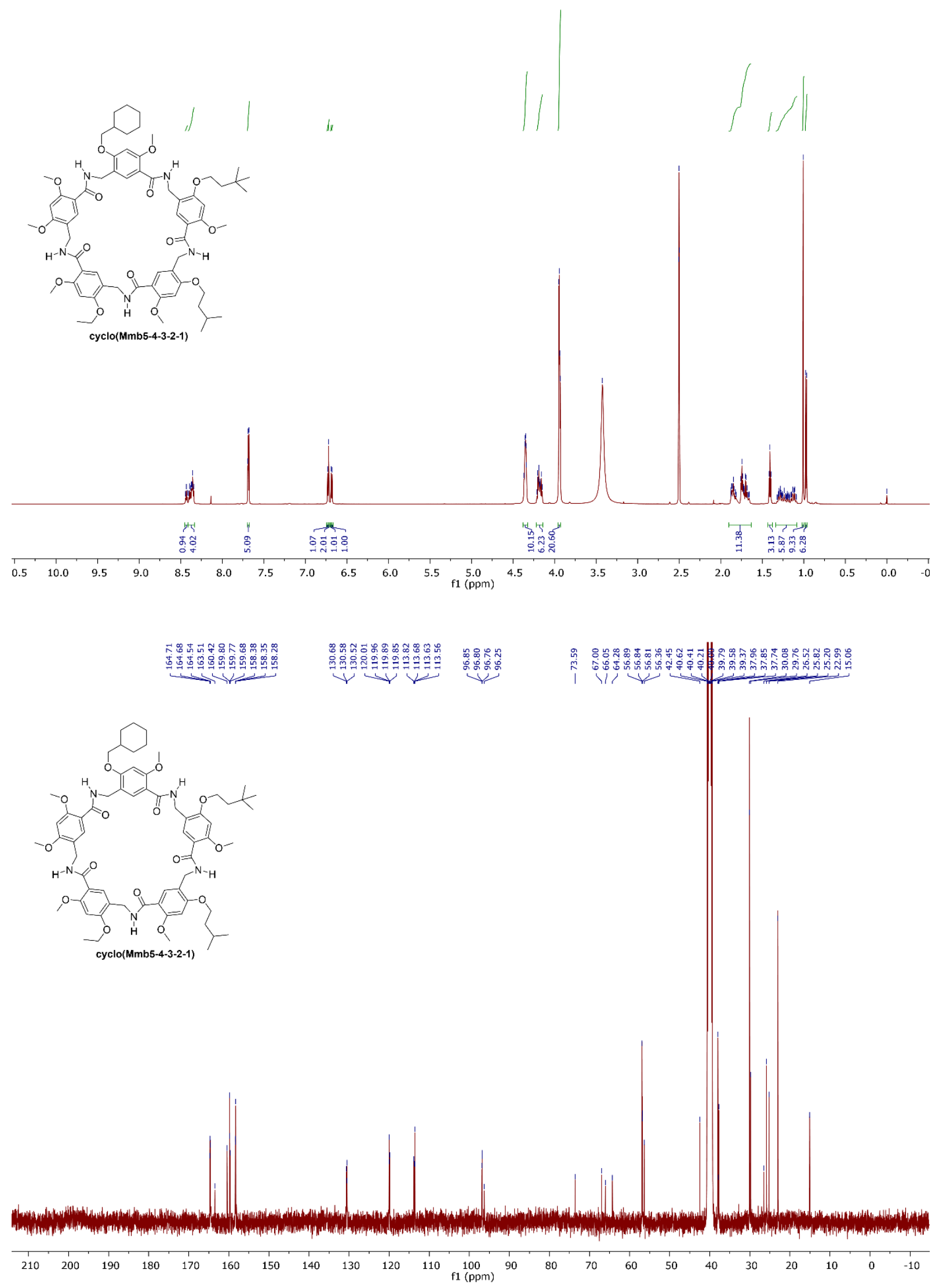

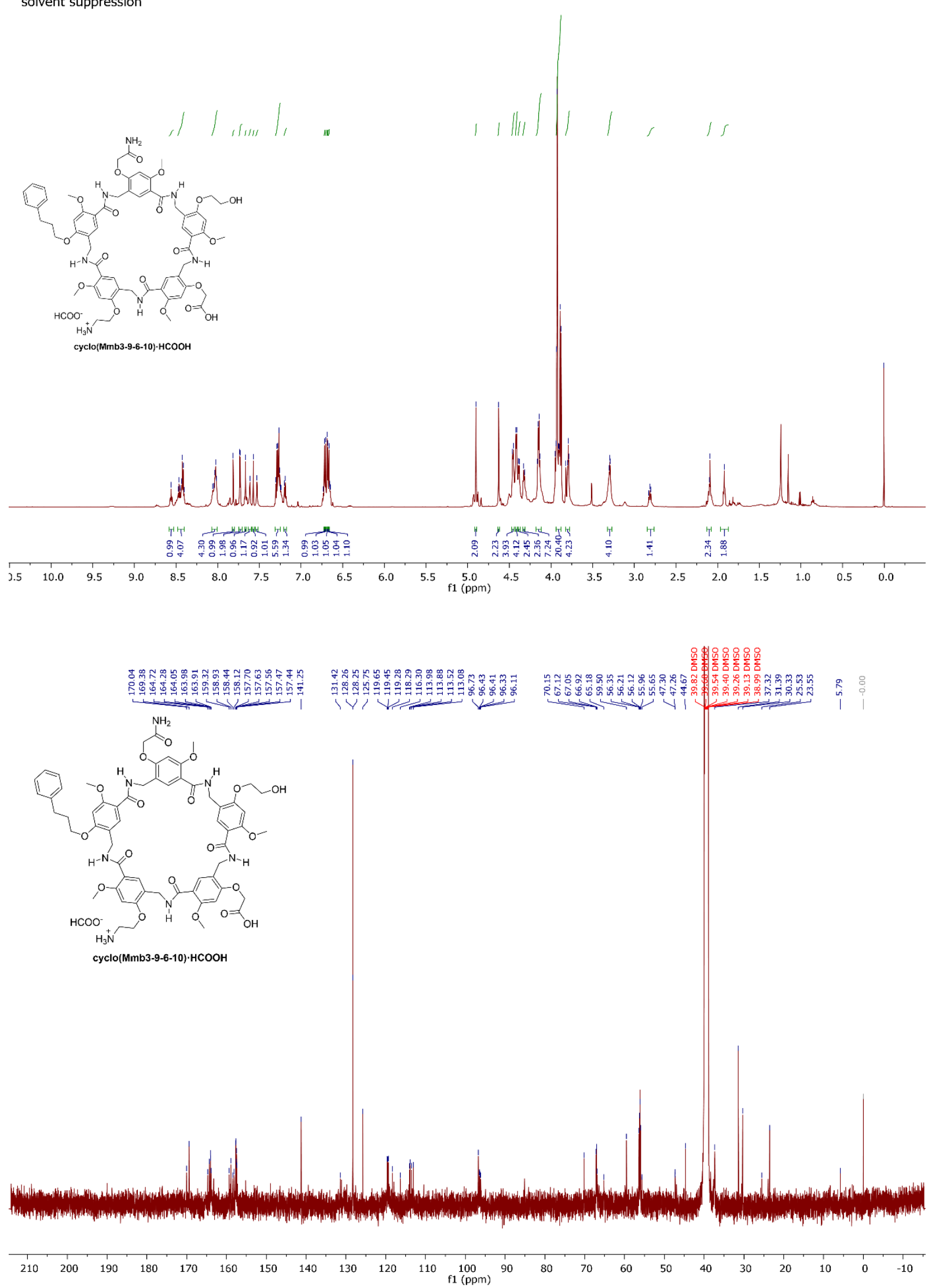

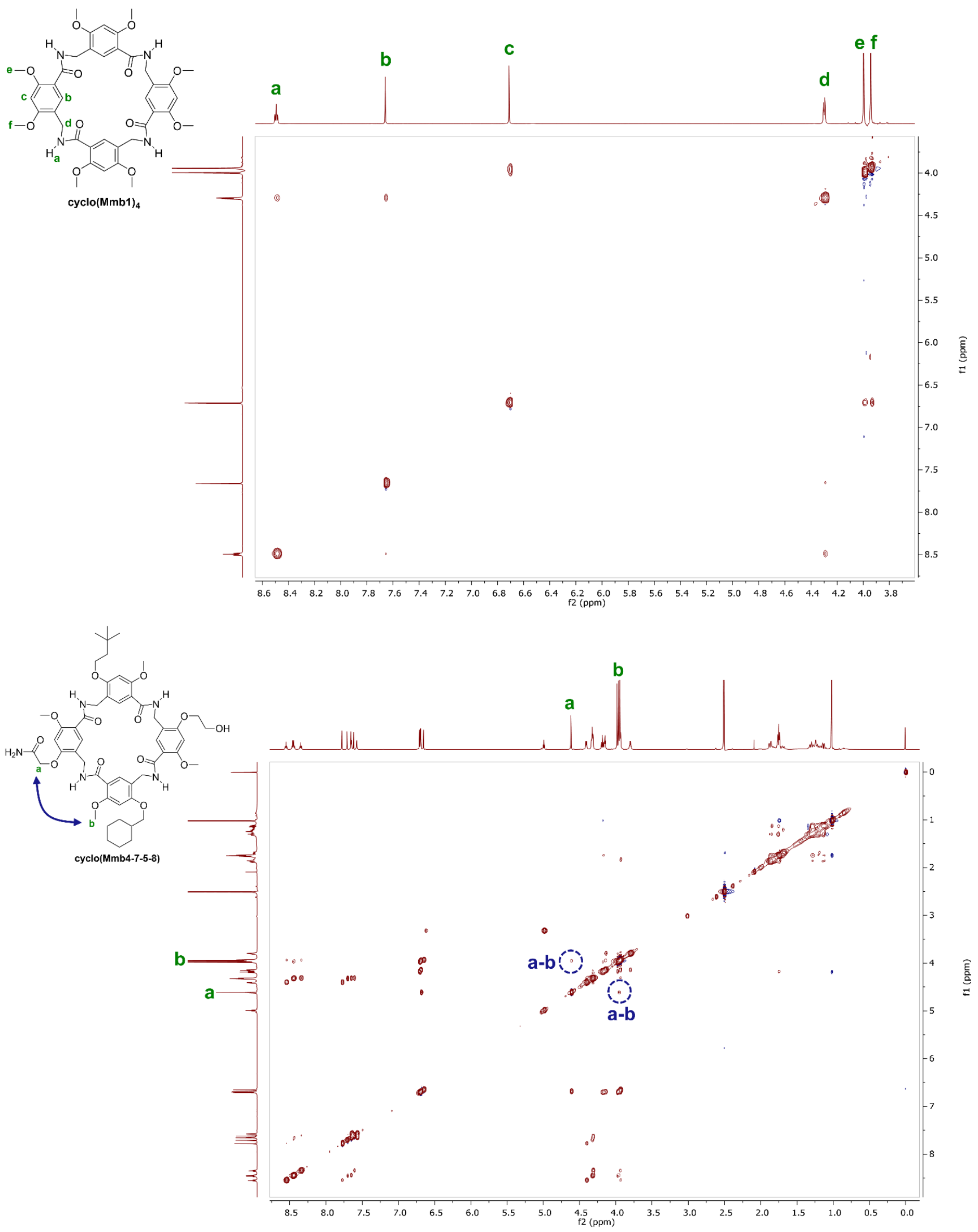

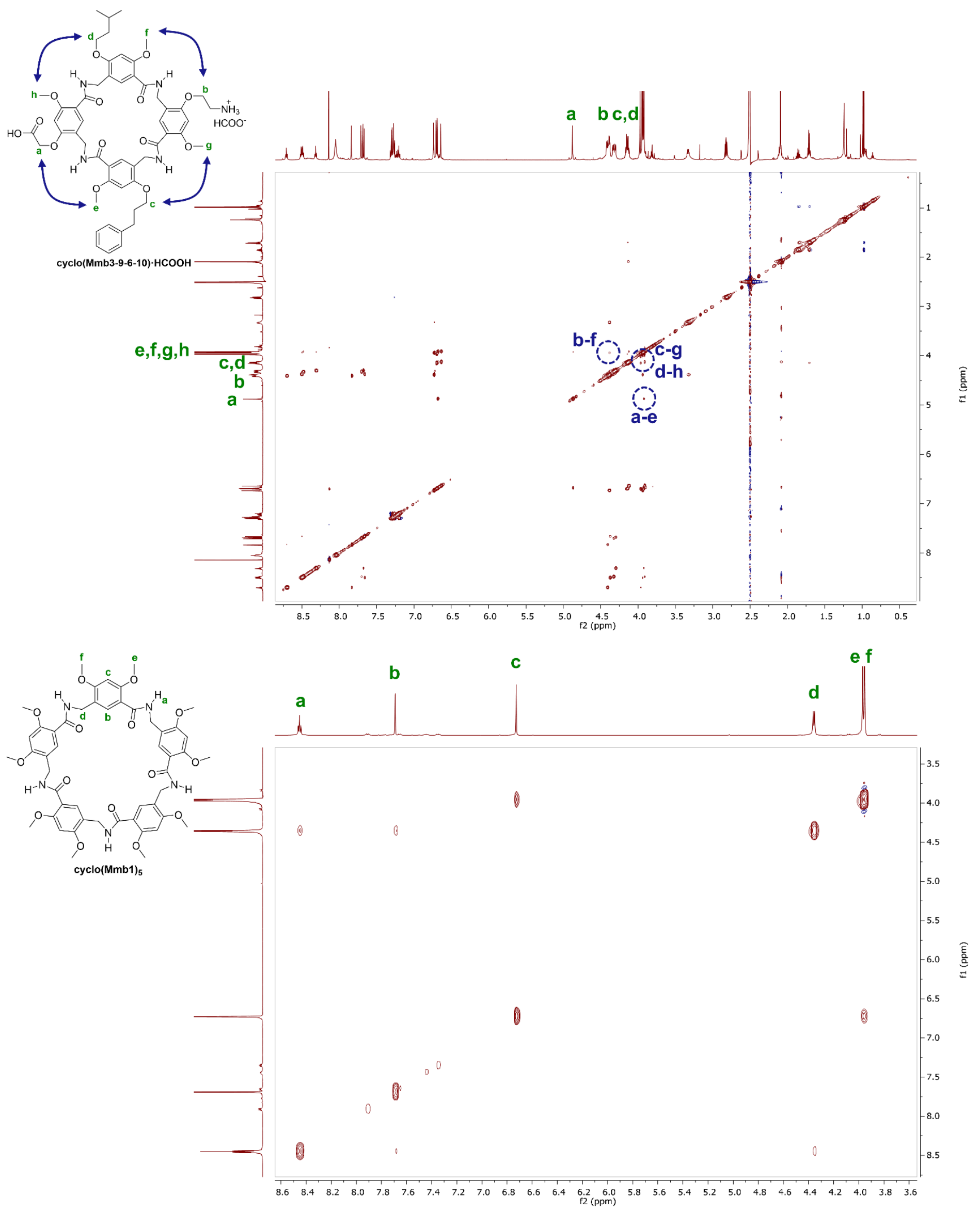

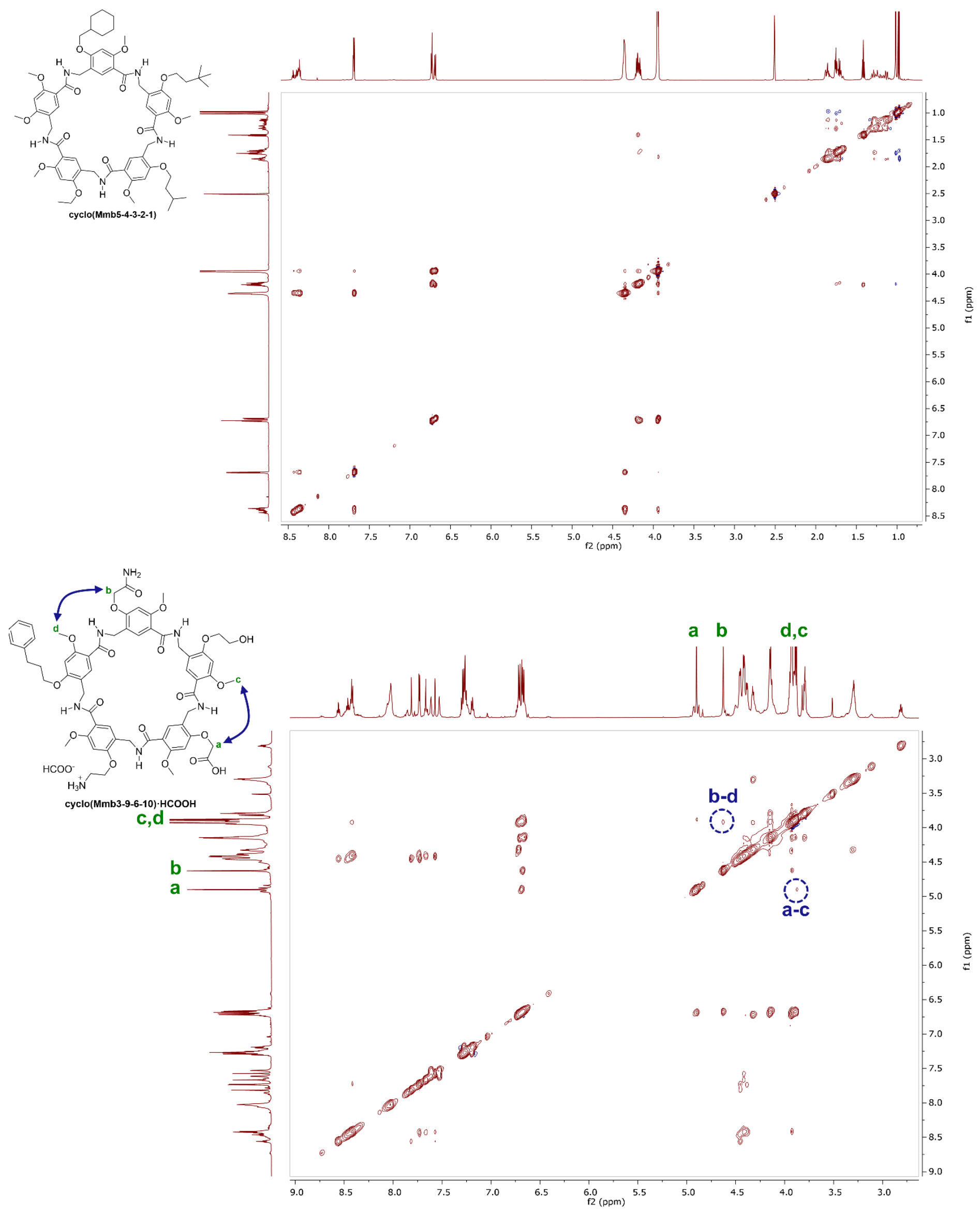


\section{X-Ray Crystallographic Analysis}

Single crystal X-ray data of complex cyclo(Mmb1)4 ${ }_{4} 6 \mathrm{HFIP} \bullet 0.85 \mathrm{MeCN}$, cyclo(Mmb1) $)_{4} \bullet \mathbf{6 H F I P} \bullet 1.04 \mathrm{H}_{2} \mathrm{O}$, and cyclo(Mmb1)5•5HFIP•MeCN•0.37H2O were recorded on a Bruker D8 APEX-II CCD system equipped with a sealed Mo tube, a graphite monochromator, and a $0.5 \mathrm{~mm}$ MonoCap collimator. All datasets were collected at $100 \mathrm{~K}$, which was controlled by an Oxford CryoSystems 700+ Cooler. The datasets were processed with the APEX2 software for reduction and cell refinement ${ }^{[\mathrm{S} 1]}$. Multi-scan absorption corrections were applied by the SCALE program for the area detector. All structures were solved by intrinsic phasing methods (SHELXT) ${ }^{[\mathrm{S} 2]}$ and the structure models were completed and refined using the full-matrix least-square methods on $F^{2}$ (SHELXL) ${ }^{[\mathrm{S} 3]}$. Non-hydrogen atoms in the structures were refined with anisotropic displacement parameters. Hydrogen atoms on carbons were placed in idealized positions $(\mathrm{C}-\mathrm{H}=0.95-1.00 \AA)$ and included as riding with $U$ iso $(\mathrm{H})=1.2$ or $1.5 \mathrm{Ueq}($ non- $\mathrm{H})$. Hydrogen atoms on nitrogens or oxygens were found from the difference Fourier electron density maps and refined isotropically. The selected crystallographic parameters of three structures were listed in Table S1. The crystallographic information files (CIFs) including the HKL and RES data were deposited to the Cambridge Crystallographic Data Centre (CCDC). The reference numbers are listed in the Table S1. 


\section{A) cyclo(Mmb1) $)_{4} \cdot 6 \mathrm{HFIP} \cdot 0.85 \mathrm{MeCN}$}
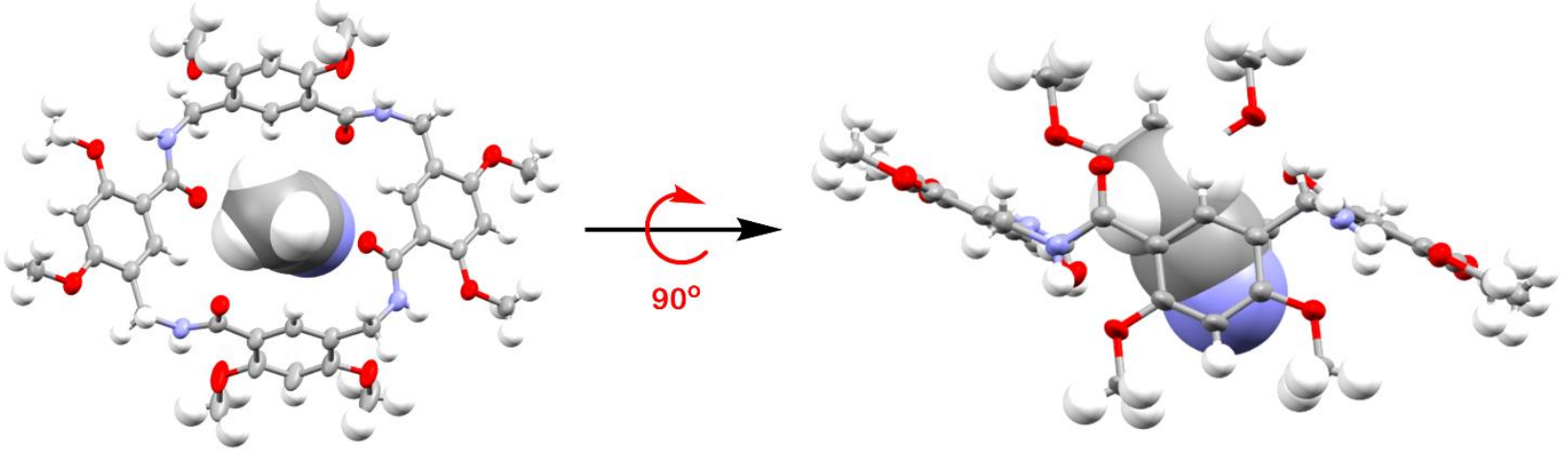

B) cyclo $(\mathrm{Mmb} 1)_{4} \cdot 6 \mathrm{HFIP} \cdot 1.04 \mathrm{H}_{2} \mathrm{O}$
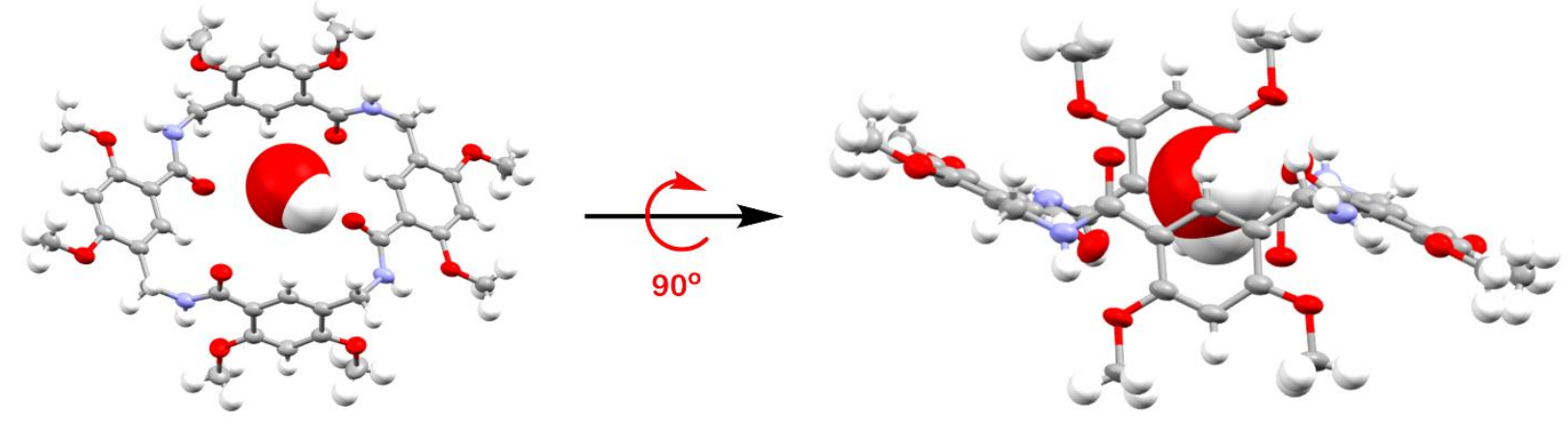

C) cyclo(Mmb1) $)_{5} \cdot 5 \mathrm{HFIP} \cdot \mathrm{MeCN} \cdot 0.37 \mathrm{H}_{2} \mathrm{O}$
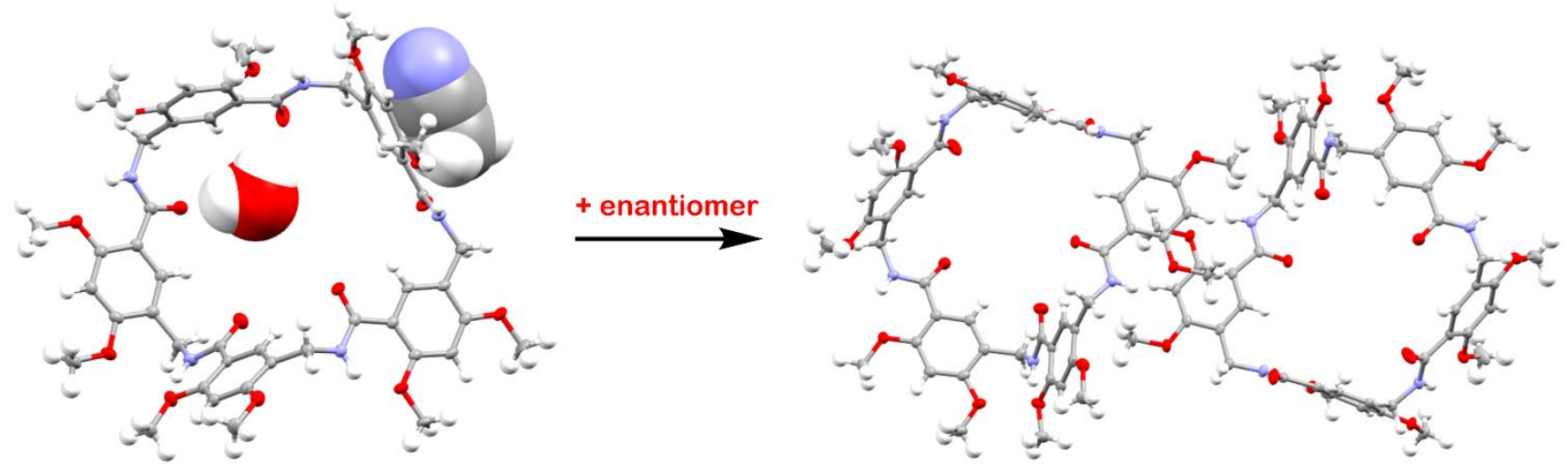

Figure S4. A) crystal structure of cyclo(Mmb1) $4 \cdot 6 \mathrm{HFIP} \bullet 0.85 \mathrm{MeCN}$ with macrocycle atoms represented as thermal ellipsoids; HFIP solvent molecules are omitted to show the location of the guest acetonitrile molecule. B) crystal structure of cyclo(Mmb1) $)^{\bullet} 6 \mathrm{HFIP} \bullet 1.04 \mathrm{H}_{2} \mathrm{O}$ with macrocycle atoms represented as thermal ellipsoids; HFIP solvent molecules are omitted to show the location of the guest water molecule. C) crystal structure of $\mathbf{c y c l o}(\mathbf{M m b 1})_{5} \cdot 5 \mathrm{HFIP} \cdot \mathrm{MeCN} \bullet 0.37 \mathrm{H}_{2} \mathrm{O}$ with macrocycle atoms represented as thermal ellipsoids; HFIP solvent molecules are omitted in the left structure to show the location of the acetonitrile and water molecules (left) and all solvent molecules are omitted in the right structure to show the interaction of enantiomers. 
Table S1. Selected crystal data for cyclo(Mmb1) $4 \cdot 6 \mathrm{HFIP} \bullet 0.85 \mathrm{MeCN}$, cyclo(Mmb1)4 $\bullet \cdot 6 \mathrm{HFIP} \bullet 1.04 \mathrm{H}_{2} \mathrm{O}$, and

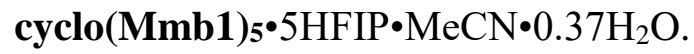

\begin{tabular}{|c|c|c|c|}
\hline Complex & $\begin{array}{l}\text { cyclo }(\mathrm{Mmb} 1)_{4} \\
\bullet 6 \mathrm{HFIP} \bullet 0.85 \mathrm{MeCN}\end{array}$ & $\begin{array}{l}\text { cyclo }(\mathrm{Mmb} 1)_{4} \\
\bullet 6 \mathrm{HFIP} \cdot 1.04 \mathrm{H}_{2} \mathrm{O}\end{array}$ & $\begin{array}{l}\text { cyclo(Mmb1) } \\
\cdot 5 \mathrm{HFIP} \bullet \mathrm{MeCN} \bullet 0.37 \mathrm{H}_{2} \mathrm{O}\end{array}$ \\
\hline X-ray code & 17adh4h & 17adh5h & 17adh15h \\
\hline CCDC no. & 1939770 & 1939771 & 1939772 \\
\hline Formula & $\mathrm{C}_{58} \mathrm{H}_{56} \mathrm{~F}_{36} \mathrm{~N}_{4} \mathrm{O}_{18} \cdot 0.85(\mathrm{MeCN})$ & $\mathrm{C}_{58} \mathrm{H}_{56} \mathrm{~F}_{36} \mathrm{~N}_{4} \mathrm{O}_{18} \cdot 1.04\left(\mathrm{H}_{2} \mathrm{O}\right)$ & $\mathrm{C}_{67} \mathrm{H}_{68} \mathrm{~F}_{30} \mathrm{~N}_{6} \mathrm{O}_{20} \cdot 0.37\left(\mathrm{H}_{2} \mathrm{O}\right)$ \\
\hline Formula weight & 1816.08 & 1799.87 & 1853.94 \\
\hline Crystal habit & colorless plate & colorless prism & colorless prism \\
\hline Crystal size (mm) & $0.10 \times 0.22 \times 0.26$ & $0.18 \times 0.43 \times 0.47$ & $0.29 \times 0.41 \times 0.54$ \\
\hline Crystal system & Monoclinic & Monoclinic & Triclinic \\
\hline Space group (no.) & $C 2 / c(15)$ & $C 2 / c(15)$ & $P \overline{1}(2)$ \\
\hline$a(\AA)$ & $16.1386(7)$ & $16.1707(18)$ & $13.1451(6)$ \\
\hline$b(\AA ̊)$ & $20.9904(10)$ & $20.903(2)$ & $14.2730(7)$ \\
\hline$c(\AA ̊)$ & $22.4778(10)$ & $22.140(2)$ & $22.4475(10)$ \\
\hline$\alpha\left({ }^{\circ}\right)$ & 90 & 90 & $90.3358(8)$ \\
\hline$\beta\left({ }^{\circ}\right)$ & $106.7555(8)$ & $106.1667(13)$ & $102.4488(9)$ \\
\hline$\gamma\left({ }^{\circ}\right)$ & 90 & 90 & $107.4119(8)$ \\
\hline$V\left(\AA^{3}\right)$ & $7291.2(6)$ & 7187.9(14) & $3912.9(3)$ \\
\hline$Z$ & 4 & 4 & 2 \\
\hline$D_{c}\left(\mathrm{~g} \mathrm{~cm}^{-3}\right)$ & 1.654 & 1.663 & 1.574 \\
\hline$\mu\left(\mathrm{mm}^{-1}\right)$ & 0.179 & 0.181 & 0.160 \\
\hline$F(000)$ & 3675 & 3642 & 1891 \\
\hline Total reflections & 59899 & 46017 & 74449 \\
\hline Unique reflections & 7176 & 7359 & 16097 \\
\hline$R_{\text {int }}$ & 0.0348 & 0.0305 & 0.0285 \\
\hline$R_{1}^{\mathrm{a}}[I>2 \sigma(I)]$ & 0.0819 & 0.0602 & 0.0720 \\
\hline$w R_{2}{ }^{b}$ (all data) & 0.2548 & 0.1839 & 0.1976 \\
\hline GOF (all data) & 1.097 & 1.071 & 1.084 \\
\hline
\end{tabular}

${ }^{\mathrm{a}} R_{1}=\sum\left\|F_{o}|-| F_{c}\right\| / \sum\left|F_{o}\right| ;{ }^{\mathrm{b}} w R_{2}=\left\{\sum\left[w\left(F_{o}^{2}-F_{c}^{2}\right)^{2}\right] / \sum\left[w\left(F_{o}^{2}\right)^{2}\right]\right\}^{1 / 2}$

\section{References}

[S1] APEX2 (version 2014.11.0). Program for Bruker CCD X-ray Diffractometer Control and Data Analysis BAI, Madison, WI, (2014).

[S2] G. M. Sheldrick (2014). SHELXT - Integrated space-group and crystal-structure determination. Acta Crystallogr. Sect. A, 2014, 71, 3-8.

[S3] G. M. Sheldrick (2008). A short history of SHELX. Acta Crystallogr. Sect. A, 2008, 64, 112-122. 